\title{
What does inflation really predict?
}

\author{
Max Tegmark \\ Dept. of Physics, Massachusetts Institute of Technology, Cambridge, MA 02139, USA \\ Department of Physics, University of Pennsylvania, Philadelphia, PA 19104, USA
}

(Dated: February 2, 2008.)

\begin{abstract}
If the inflaton potential has multiple minima, as may be expected in, e.g., the string theory "landscape", inflation predicts a probability distribution for the cosmological parameters describing spatial curvature $\left(\Omega_{\text {tot }}\right)$, dark energy $\left(\rho_{\Lambda}, w\right.$, etc. $)$, the primordial density fluctuations $\left(Q, n_{s}\right.$, $d n_{s} / d \ln k$, etc.) and primordial gravitational waves $\left(r, n_{t}\right.$, etc.). We compute this multivariate probability distribution for various classes of single-field slow-roll models, exploring its dependence on the characteristic inflationary energy scales, the shape of the potential $V$ and and the choice of measure underlying the calculation. We find that unless the characteristic scale $\Delta \phi$ on which $V$ varies happens to be near the Planck scale, the only aspect of $V$ that matters observationally is the statistical distribution of its peaks and troughs. For all energy scales and plausible measures considered, we obtain the predictions $\Omega_{\mathrm{tot}} \approx 1 \pm 10^{-5}, w=-1$ and $\rho_{\Lambda}$ in the observed ballpark but uncomfortably high. The high energy limit predicts $n_{s} \approx 0.96, d n_{s} / d \ln k \approx-0.0006, r \approx 0.15$ and $n_{t} \approx-0.02$, consistent with observational data and indistinguishable from eternal $V \propto \phi^{2}$ inflation. The low-energy limit predicts 5 parameters but prefers larger $Q$ and redder $n_{s}$ than observed. We discuss the coolness problem, the smoothness problem and the pothole paradox, which severely limit the viable class of models and measures. Predictions insensitive to pre-inflationary conditions can arise either from eternal inflation attractor behavior or from anthropic selection effects probing merely a tiny non-special part of the initial distribution. We argue that these two mechanisms are severely challenged by the coolness problem and the smoothness problem, respectively. Our findings bode well for detecting an inflationary gravitational wave signature with future CMB polarization experiments, with the arguably best-motivated single-field models favoring the detectable level $r \sim 0.03$.
\end{abstract}

\section{INTRODUCTION}

The spectacular recent progress in observational cosmology has produced measurements of about a dozen cosmological parameters [1-3] as summarized in Table 1 and Figure 1 (vertical bands). This observational progress is in stark contrast to the theoretical situation: we still lack a firm a priori prediction for any one of them. Inflation is promising in potentially being able to explain a strikingly large fraction of these numbers: 8 of the 11, including (as we will argue) $\rho_{\Lambda}$ and $w$, the only holdouts being the baryon parameter $\xi_{\mathrm{b}}$ (awaiting an understanding of baryogenesis), the dark matter parameter $\xi_{\mathrm{c}}$ (perhaps awaiting an understanding of supersymmetry parameters

Table 1: Constraints on fundamental cosmological parameters computed from WMAP+SDSS as in $[2,3]$. Inflation may predict the first 8 . We have replaced the conventional parameters

$\left(\Omega_{\Lambda}, \Omega_{b}, \Omega_{\mathrm{cdm}}, \Omega_{\nu}\right)$ by the less anthropocentric ones $\left(\rho_{\Lambda}, \xi_{\mathrm{b}}, \xi_{\mathrm{c}}, \xi_{\nu}\right)$ since the latter do not depend on the observing epoch.

\begin{tabular}{|l|ll|}
\hline $\mathrm{p}$ & Meaning & Measured value \\
\hline$\Omega_{\text {tot }}$ & Spatial curvature & $1.01 \pm 0.02$ \\
$\rho_{\Lambda}$ & Dark energy density & $(9.3 \pm 2.5) \times 10^{-124} m_{\mathrm{Pl}}^{4}$ \\
$w$ & Dark energy equation of state & $-1 \pm 0.1$ \\
$Q$ & Scalar fluctuation amplitude $\delta_{H}$ on horizon & $(2.0 \pm 0.2) \times 10^{-5}$ \\
$n_{S}$ & Scalar spectral index & $0.98 \pm 0.02$ \\
$\alpha$ & Running of spectral index $d n_{s} / d \ln k$ & $|\alpha| \lesssim 0.01$ \\
$r$ & Tensor-to-scalar ratio $\left(Q_{t} / Q\right)^{2}$ & $\sim 0.36$ \\
$n_{t}$ & Tensor spectral index & Unconstrained \\
\hline$\xi_{\mathrm{b}}$ & Baryon mass per photon $\rho_{\mathrm{b}} / n_{\gamma}=m_{\mathrm{p}} \eta$ & $(0.60 \pm 0.03) \mathrm{eV}$ \\
$\xi_{\mathrm{c}}$ & CDM mass per photon $\rho_{\mathrm{c}} / n_{\gamma}$ & $(3.3 \pm 0.2) \mathrm{eV}$ \\
$\xi_{\nu}$ & Neutrino mass per photon $\rho_{\nu} / n_{\gamma}=\frac{3}{11} \sum m_{\nu_{i}}<0.11 \mathrm{eV}$ \\
\hline
\end{tabular}

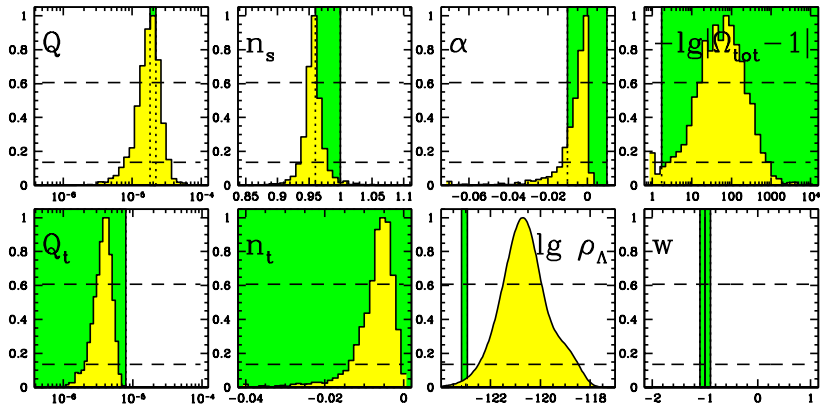

FIG. 1: Yellow/light grey cosmological parameter distributions show inflationary predictions for our $\S 5$ example with characteristic $\phi$-scale $m_{h} \approx 2 m_{\mathrm{Pl}}, V$-scale $m_{v}=0.002 m_{\mathrm{Pl}}$ and uniform measure over initial $\phi$-values. Green/dark grey regions show observational constraints $(1 \sigma)$ from Table 1 and $[2,3] . \rho_{\Lambda}$ is in Planck units.

governing dark matter freezeout) and the neutrino parameter $\xi_{\nu}$ (awaiting an understanding of the origin of neutrino masses).

So what does inflation predict for the parameter vector

$$
\mathbf{p} \equiv\left(\Omega_{\mathrm{tot}}, \rho_{\Lambda}, w, Q, n_{s}, \alpha, r, n_{t}\right) ?
$$

The rhetorical answer is that the answer is determined by the inflaton potential $V(\phi)$, where $\phi$ is a vector of one or more scalar fields, and that the only fairly robust predictions are $\Omega_{\mathrm{tot}} \approx 1$ and the slow-roll consistency relation $r=-8 n_{t}$ - with known exceptions even to these, e.g. [4-7]. Since a bewilderingly large number of potentials have been studied in the literature (see [8-15] for recent reviews), producing predictions in pretty much ever cor- 


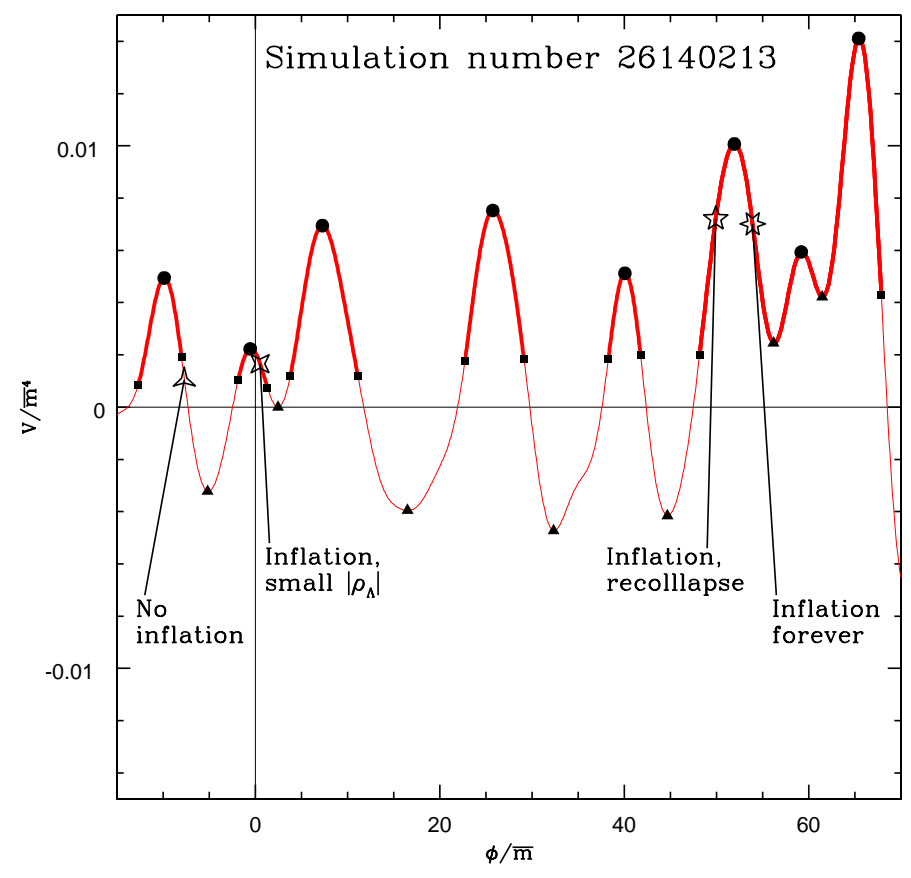

FIG. 2: A small segment of the inflaton potential $V(\phi)$ for simulation number 26140213 from $\S 5$, with vertical and horizontal energy scales $m_{h}=3 \bar{m}$ and $m_{h}=0.007 \bar{m}$, respectively $\left(\bar{m}=m_{\mathrm{Pl}} / \sqrt{8 \pi}\right)$. A half-basin of attraction stretches from a local maximum (circle) to a local minimum (triangle). The thicker curve indicates regions where the slow-roll approximation is valid, with squares indicating inflation endpoints. Many starting points $\phi$ fail to produce galaxies, either because the slow-roll approximation in invalid and there is no inflation (three-pointed star), because inflation is rapidly followed by $\rho_{\Lambda}<0$ recollapse (five-pointed star) or because inflation never ends (six-pointed star). Only a small fraction of starting points (like the four-pointed star) give inflation followed by a vacuum density $\left|\rho_{\Lambda}\right|$ near zero.

ner of the currently allowed parameter space, it is easy to come away with the impression that inflation is not very predictive and hence difficult to test or rule out.

However, this pessimistic conclusion rests on a rather dubious premise: that the way inflation occurred in our region of space is the only way that inflation occurred anywhere. This is the antithesis of the "landscape" paradigm now emerging from string theory, with an extremely complicated potential with perhaps $10^{300}$ different minima [16-29], c.f. [30, 31].

Whatever the correct fundamental theory may turn out to be, one can easily imagine it giving rise to an inflaton potential $V(\phi)$ with multiple minima, as in the example shown in Figure 2. As long as $\phi$ takes on a range of values early on ${ }^{1}$, different post-inflationary domains

\footnotetext{
${ }^{1}$ Having $\phi$ take on a range of values early on is easy to arrange. The same quantum fluctuation process responsible for generating our observed density fluctuations gives rise to the familiar
}

will correspond to $\phi$ having rolled down into different minima, and so the parameter values we measure will depend on which domain we inhabit. In other words, what is traditionally referred to as the inflaton potential is merely the basin of attraction around our particular local minimum $\phi_{0}$. Our observed cosmological constant will be $\rho_{\Lambda}=V\left(\phi_{0}\right)$, our gravity wave amplitude will be given by $V\left(\phi_{1}\right)$ at the point $\phi_{1}$ about $55 e$-foldings before our inflation ended $[36,37], n_{s}$ will be determined by the first and second derivatives of $\ln V$ at at $\phi_{1}$, and so on. Even if the potential has only one minimum, parameters such as $n_{s}$ will depend on the direction from which one rolls down to this minimum (from the left or from the right for single-field inflation like in Figure 2, or from a continuum of directions for multi-field inflation where $\phi$ is a vector).

If one or more explicit effective potentials $V(\phi)$ emerge from string theory or some other fundamental theory [38], then it is clearly worthwhile computing or estimating the corresponding 8-dimensional parameter probability distribution $f_{p}(\mathbf{p})$ (Figure 1 shows an example), since this will provide a powerful observational test of the theory. The purpose of the present paper is to lay the groundwork for such a calculation in the context of classic slow-roll inflation, computing $f_{p}(\mathbf{p})$ for various classes of randomly generated potentials and exploring its dependence both on the potential $V(\phi)$ and on the choice of measure underlying the calculation. We will see that a number of general conclusions can be drawn rather independently of the detailed nature of the potential $V(\phi)$, depending mainly on energy scales. For related discussions of such issues in the context in inflationary flow equations, see [39-41].

We find that the issue of what measure to use when sampling $4 \mathrm{D}$ spacetime is no less important than the issue of what potential $V(\phi)$ to use. This measure problem cannot be brushed aside as irrelevant philosophy, since the measure dramatically affects the testable prediction for $f_{p}(\mathbf{p})$, and we will argue that some plausiblesounding and oft-discussed measures like synchronous volume-weighting are already ruled out by observation. There is some correct measure that nature subscribes to, and we need to figure out which one it is, just as was successfully done in the past for the measures allowing us to compute probabilities in statistical mechanics and quantum physics.

The rest of this paper is organized as follows. Section II briefly summarizes how the potential $V(\phi)$ affects what we observe, emphasizing that modulo a slight

diffusion process that can drive $\phi$ uphill as well as downhill [32], effectively populating all $\phi$-values in a quantum superposition. Decoherence [33] then insures that we can for all practical purposes treat $\phi$ as having different classical values in different spatial regions $[34,35]$. Alternatively, an early hot state with energy in excess of typical $V$-values would naturally populate all $\phi$-values. 
caveat in the multidimensional case, all inflationally observables (including the eight inflation-related parameters in Table 1) are determined by the cosmic density history $\rho(a)$ alone, independent of the details of $V(\phi)$. Sections III and IV discuss how the measure affects the parameter probability distribution $f_{p}(\mathbf{p})$, i.e., what we should expect to observe given $V(\phi)$, classifying various plausible and ruled-out measure candidates. Section V presents a suite of numerical experiments exploring the effect of inflationary energy scales, and Section VI shows how to compute $f_{p}(\mathbf{p})$ from these results when including selection effects. Section VII complements our numerical exploration with a suite of analytic calculations, and Section VIII summarizes our conclusions. Appendix A and $\mathrm{B}$ give technical details of how we compute $\mathbf{p}$ from $V$.

\section{HOW THE POTENTIAL AFFECTS WHAT WE OBSERVE}

Given the measure subtleties discussed in the next section, it is crucial to be clear on what question we are asking. In this section, we address the following question: If we live in a part of spacetime where the inflaton has evolved as $\phi(a)$ and the density has evolved as $\rho(a)$, then what do we observe? This is mainly a review of standard material, organized to clarify how in many cases, all observables can be calculated directly from $\rho(a)$ alone, without needing to know $\phi(a)$ or the inflaton potential.

We wish to make predictions for four basic observables:

1. The curvature of space given by $\Omega_{\text {tot }}$.

2. The dark energy density as a function of cosmic scale factor, $\rho_{\Lambda}(a)$.

3. The primordial power spectrum of gravitational waves $\delta_{H}^{T}(k)$.

4. The primordial power spectrum of adiabatic density fluctuations $\delta_{H}(k)$.

It is popular to approximate the three cosmological functions $\rho_{\Lambda}(a), \delta_{H}^{T}(k)^{2}$ and $\delta_{H}(k)^{2}$ by linear and quadratic functions in log-log space, parametrized by parameters 2-8 from Table 1:

$$
\begin{aligned}
\rho_{\Lambda}(a) & =\rho_{\Lambda} \times\left(a / a_{0}\right)^{-3(1+w)}, \\
\delta_{H}^{T}(k)^{2} & =Q^{2} \times r(k / H)^{n_{t}}, \\
\delta_{H}(k)^{2} & =Q^{2} \times(k / H)^{n_{s}-1+\alpha \ln (k / H) / 2},
\end{aligned}
$$

where $H \equiv \dot{a} / a$ is the current Hubble parameter, so that the wavenumber $k=H$ corresponds to a fluctuation on the current horizon scale ${ }^{23}$. Here and throughout, we will

\footnotetext{
2 The horizon is $c / H-$ throughout this paper, we use standard particle physics units where $\hbar=c=k_{b}=1$, so the Planck mass is simply $m_{\mathrm{Pl}}=G^{-1 / 2}$.

${ }^{3}$ For simplicity, we have defined the normalizations $(Q, r Q)$ and
}

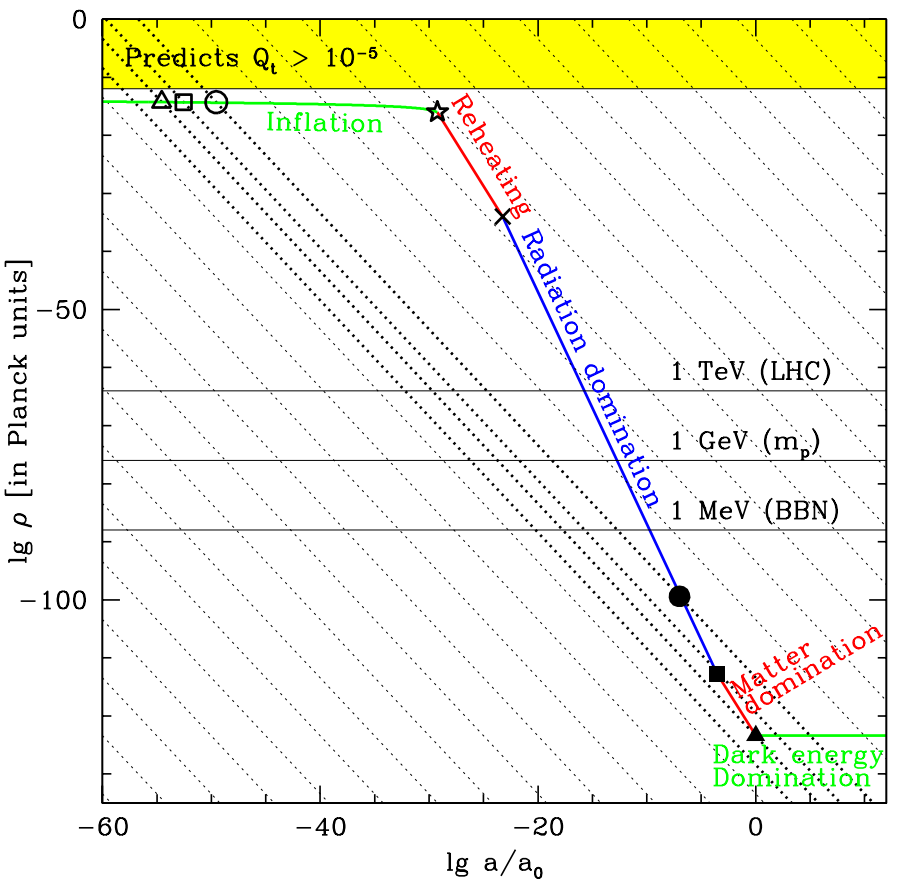

FIG. 3: The spatial curvature parameter $\Omega_{\text {tot }}$, the primordial gravitational wave power spectrum $\delta_{H}^{T}(k)$ and the dark energy function $\rho_{\Lambda}(a)$ are all determined by the single curve $\rho(a)$ above, as is the primordial density fluctuation spectrum $\delta_{H}(k)$ in many cases. Dotted diagonals of slope -2 are lines of constant comoving horizon size $a / H^{-1}=\dot{a}$, governing when fluctuations exit end reenter the horizon. Inflation $(\ddot{a}>0)$ is when the cosmic density history $\rho(a)$ has a shallower slope than these diagonals, $d \ln \rho / d \ln a>-2$, and this logarithmic slope normally becomes -3 during reheating, -4 during radiation domination, -3 during matter domination and $>-2$ now during dark energy domination. When two points lie on the same diagonal (like the two triangles), it means that that the horizon volume at the two epochs is the same comoving spatial region. $\Omega_{\text {tot }}$ is constant on these diagonals, approaching unity towards the upper right, and unless the $\rho(a)$ curve crosses the leftmost heavy diagonal, we should observe curvature $\left|\Omega_{\mathrm{tot}}-1\right|>10^{-5}$. The dark energy function $\rho_{\Lambda}(a)$ is simply the curve $\rho(a)$ at late times minus the matter contribution. The primordial gravitational wave power spectrum is simply the curve $\rho(a)$ at early times, rescaled vertically (by a factor of two since $\delta_{H}^{T}(k) \propto \rho^{1 / 2}$ ) and horizontally (mapping $a$ into $k$ matching horizon exit and reentry with the diagonal lines, as with the pairs of triangles, squares and circles). For single-field inflation and many multi-field cases, the primordial density fluctuation spectrum is determined from the curve $\rho(a)$ and its derivative by $\delta_{H}(k) \propto \rho^{1 / 2}(-d \ln \rho / d \ln a)^{-1 / 2}$.

assume the standard inflationary fluctuation-generation mechanism which produces adiabatic fluctuations - for

tilts $\left(n_{s}, n_{t}\right)$ on the horizon scale. A popular alternative convention $[1-3,42]$ is to specify them on the comoving scale corresponding to $k=0.05 / \mathrm{Mpc}$ at the present epoch - this corresponds to shifting the emphasis from, say, scales that are $N=55$ e-foldings before the end of inflation to $N \approx 50$, making only a minor difference in $\mathbf{p}$ that is irrelevant for the purposes of this paper. 
a review of alternatives, see [8-15, 43].

Figure 3 is an attempt to capture all the inflationary observables in one plot. It illustrates how the late-time behavior of $\rho(a)$ encodes the dark energy function $\rho_{\Lambda}(a)$ whereas its early-time behavior determines the curvature parameter $\Omega_{\text {tot }}$, the gravitational wave power spectrum $\delta_{H}^{T}(k)$ and, in many cases, the power spectrum of density fluctuation $\delta_{H}(k)$. Key epochs in Figure 3 are $a_{\text {start }}$ (when inflation began; $a_{\text {start }}=0$ if there was no beginning), $a_{\text {exit }}$ (open triangle, when our current Hubble volume left the horizon), $a_{\mathrm{k}}$ (when a fluctuation of wavenumber $k$ leaves the horizon; open square or circle, say), $a_{\text {end }}$ (star, when inflation ended), $a_{\text {reheat }}$ (cross, when reheating ended), $a_{\text {eq }}$ (filled square, matterradiation equality) and $a_{0}$ (filled triangle, now). Figure 3 also provides a simple graphical way of reading off bounds on the number of e-foldings $N$ between the creation of our observed horizon-scale fluctuations (open triangle) and the end of inflation (star) - most popular models give $N \sim 55 \pm 5[36,37]$.

For comparison, the ekpyrotic model $[44,45]$ has fluctuations leave the horizon during a contracting phase when we move up to the left in Figure 3 with steeper slope than -2 .

We will now summarize the calculation of the cosmological functions $\rho_{\Lambda}(a), \delta_{H}^{T}(k), \delta_{H}^{T}(k)$ and the eight inflationary parameters from Table 1 . As detailed in Appendix A, our three cosmological functions and the Hubble parameter are given in terms of $\rho(a)$ by

$$
\begin{aligned}
\rho_{\Lambda}(a) & =\rho(a)-\rho_{\gamma \mathrm{mk}}(a), \\
\delta_{H}^{T}(k) & =\sqrt{\frac{8 \rho\left(a_{\mathrm{k}}\right)}{75 \pi^{2} \bar{m}^{4}}}, \\
\delta_{H}(k) & \geq \sqrt{\frac{\rho\left(a_{\mathrm{k}}\right)}{75 \pi^{2} \bar{m}^{4}(\ln \rho)^{\prime}}} \\
H(a) & \equiv \frac{d \ln a}{d t}=\sqrt{\frac{8 \pi \rho(a)}{3 m_{\mathrm{Pl}}^{2}}}=\frac{\rho(a)^{1 / 2}}{\sqrt{3} \bar{m}},
\end{aligned}
$$

where $^{\prime} \equiv d / d N=-d / d \ln a, \rho_{\gamma \mathrm{mk}}(a)$ is the density contribution from photons, matter and curvature, the mode exit scale is

$$
a_{k} \approx \frac{a_{\text {exit }}}{H\left(a_{\text {exit }}\right)} k=\frac{\sqrt{3} \bar{m} a_{\text {exit }}}{\rho\left(a_{\text {exit }}\right)^{1 / 2}} k,
$$

$a_{\text {exit }}$ is the solution to the equation $a_{\text {exit }}^{2} \rho\left(a_{\text {exit }}\right)=$ $a_{0}^{2} \rho\left(a_{0}\right)$, and the reduced Planck mass

$$
\bar{m} \equiv \frac{m_{\mathrm{Pl}}}{\sqrt{8 \pi}} \approx \frac{m_{\mathrm{Pl}}}{5} .
$$

is defined so as to minimize the number of $8 \pi$-factors in this paper. The corresponding eight observable cosmological parameters in Table 1 are given in terms of $\rho(a)$ by (see Appendix A)

$$
\begin{aligned}
\left|\Omega_{\mathrm{tot}}-1\right| & \sim 10^{-5}+\left[\frac{a_{\text {exit }} \rho\left(a_{\text {exit }}\right)}{a_{\text {start }} \rho\left(a_{\text {start }}\right)}\right]^{2}, \\
\rho_{\Lambda} & =\lim _{a \rightarrow \infty} \rho(a) \\
w & =-1 \\
Q & \geq \sqrt{\frac{\rho\left(a_{\text {exit }}\right)}{75 \pi^{2} \bar{m}^{4}(\ln \rho)^{\prime}}} \\
n_{s} & =1-(\ln \rho)^{\prime}+\frac{(\ln \rho)^{\prime \prime}}{(\ln \rho)^{\prime}} \\
\alpha & =(\ln \rho)^{\prime}+\frac{(\ln \rho)^{\prime \prime 2}}{(\ln \rho)^{\prime 2}}-\frac{(\ln \rho)^{\prime \prime \prime}}{(\ln \rho)^{\prime}} \\
r & \leq 8(\ln \rho)^{\prime} \\
n_{t} & =-(\ln \rho)^{\prime}
\end{aligned}
$$

where $^{\prime} \equiv \frac{d}{d \ln a}$. The usual slow-roll parameters are

$$
\begin{aligned}
\epsilon & =\frac{1}{2}(\ln \rho)^{\prime} \\
\eta & =(\ln \rho)^{\prime}+\frac{(\ln \rho)^{\prime \prime}}{2(\ln \rho)^{\prime}}
\end{aligned}
$$

in terms of which we have the standard relations

$$
\begin{aligned}
n_{s} & =1-6 \epsilon+2 \eta, \\
n_{t} & =-2 \epsilon, \\
r & \leq 16 \epsilon \\
\alpha & =16 \epsilon \eta-24 \epsilon^{2}-2 \xi_{2} .
\end{aligned}
$$

As detailed in Appendix A, the inequalities (14), (17) and (24) become equalities for single-field inflation and also to good approximation for most multi-field models. Similarly, the expressions for $n_{s}$ and $\alpha$ are exact for single-field models and usually good approximations for multi-field models.

In addition to determining these numerical parameters, the $\rho(a)$-curve in Figure 3 readily allows qualitative conclusions to be read off: To solve the horizon problem, the curve must cross the dotted diagonal that runs through the filled triangle, and equation (11) shows that to solve the flatness problem, it must also cross the diagonal line lying about a factor of 10 further to the left.

\section{HOW THE MEASURE AFFECTS WHAT WE OBSERVE}

In this Section, we discuss how to confront an inflation model with observational data, i.e., how to compute the theoretically predicted probability distribution $f_{p}(\mathbf{p})$ for the cosmological parameters once $V(\phi)$ is known. 


\section{A. Overview of the measure problem}

Inflation helps create an interestingly complex spacetime, one or more parts of which contain observers like us. Loosely speaking, the probability distribution $f_{p}(\mathbf{p})$ for the cosmological parameters is simply an eightdimensional histogram, reflecting the distribution of parameters measured by the different observers. Let us now make this more rigorous. To make inflation a fully specified and testable theory from which $f_{p}(\mathbf{p})$ can be computed, three things need to be specified:

1. the choice of reference objects (say points, protons, planets or observers),

2. the order in which to count them (this matters when there are infinitely many),

3. the initial conditions (irrelevant in some cases).

We will refer to the specification of these three things as a choice of measure. We will first summarize these three issues briefly, then revisit them in greater detail.

\section{Reference objects}

The first of the three choices is the most obvious and straightforward to deal with, so we will not dwell on it here - see $[46,47]$ for a detailed discussion. The resulting parameter probability distribution $f_{p}(\mathbf{p})$ depends strongly on whether the reference objects are points, protons, dark matter halos, galaxies, stars or self-aware observers (whatever that means), so the key point is simply to be explicit about what reference objects are used and to quantify the sensitivity of the results to this choice - we will explore various choices below, in Section VI. Mathematically, this is simply standard use of conditional probabilities: we compute the probability distribution for the parameters $\mathbf{p}$ measured from a spacetime point given various constraints, say that it is the location of a galaxy. ${ }^{4}$

\footnotetext{
${ }^{4}$ Discussions of selection effects often turn heated when somebody mentions the "A-word", anthropic. The author feels that discussions of the so-called anthropic principle [63-66] have generated more heat than light, largely because of a preponderance of different and mutually incompatible definitions and interpretations of what it means. The author is not aware of anybody disagreeing with what might be termed MAP, the "minimalistic anthropic principle": When testing fundamental theories with observational data, ignoring selection effects can give incorrect conclusions. This is all we subscribe to in the present paper. We wish to test any fundamental theory that predicts $V(\phi)$ by calculating its cosmological parameter predictions $f_{p}(\mathbf{p})$, and including selection effects is clearly not optional. The question of precisely which selection effects to use (i.e., what reference objects to condition on) is difficult and not settled. The appropriate response to this is clearly not to give up and ignore selection effects altogether, but rather to explore a range of options and quantify the extent to which the choice affects the results.
}

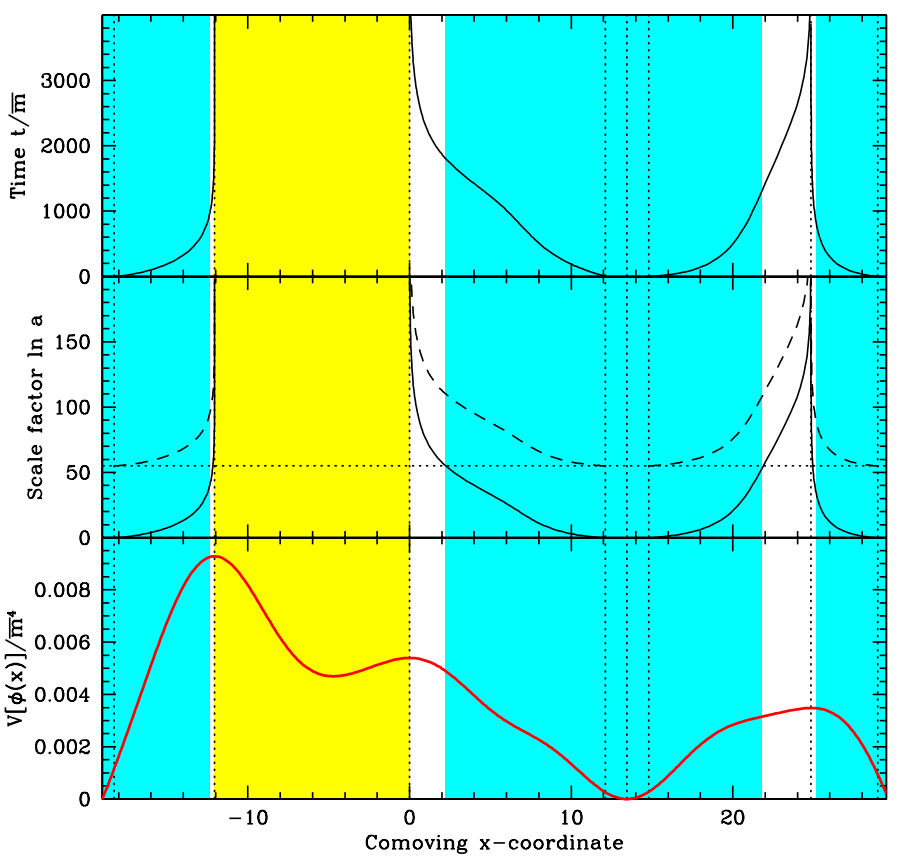

FIG. 4: Spacetime foliation examples. Solid curves (top and middle panels) show infinite hypersurfaces corresponding to the end of inflation, starting at $t=0, a=1$ with an inflaton field $\phi(\mathbf{r})=\varepsilon x$ with $\varepsilon$ so small that spatial gradients are negligible. Dashed curve (middle panel) corresponds to the present density. The four shaded regions of comoving space fail to produce any Hubble volumes resembling ours, either because inflation never ends (yellow/light grey) or because inflation never starts or lasts less than 55 e-foldings (cyan/grey). Lower panel shows the inflaton potential used, with vertical dotted lines showing maxima, minima and inflation endpoints.

\section{Ordering}

Figure 4 illustrates what we will call the ordering problem. We have chosen our reference objects (protons, say), and need to locate all of them in the spacetime manifold. Inflation creates photons and other particles on spatial hypersurfaces corresponding to the end of inflation, illustrated by the U-shaped curves in the figure. As detailed in Section VII, each of these hypersurfaces typically has infinite physical volume, producing infinitely many reference objects. Such a hypersurface corresponds to a particular basin of attraction in $V(\phi)$, i.e., everywhere in this comoving volume, the inflaton field $\phi$ rolls down to the same local minimum $\phi_{0}$. We will usually refer to such spatial domains as thermalized regions, pocket universes [48-50] or "pockets" for brevity — other terms for them include bubble universes and Level I multiverses $[51] .^{5}$ If we order the reference objects $1,2,3, \ldots$ and let

\footnotetext{
${ }^{5}$ Note that there is not a one-to-one correspondence between basins and pockets. Each pocket corresponds to exactly one
} 
$\mathbf{p}_{i}$ denote the parameters observed from the $i^{t h}$ object, then we can compute the probability distribution as

$$
f_{p}(\mathbf{p}) \equiv \lim _{n \rightarrow \infty} \frac{1}{n} \sum_{i=1}^{n} \delta\left(\mathbf{p}-\mathbf{p}_{i}\right)
$$

The crux is that when there are infinitely many objects [48-50], the ordering affects the answer! For example, suppose we order the objects by increasing physical distance from some given point $\mathbf{r}$ at fixed time (defined as fixed cosmic density). This may sound like an innocent and reasonable algorithm, since it is equivalent to the familiar procedure of averaging in a spherical volume and then letting the sphere radius approach infinity. However, since the pockets (thermalized regions) are infinite, this ordering will only include objects within the same pocket that contains the reference point $\mathbf{r}$, giving zero statistical weight to the reference objects in all other pockets.

This illustrates a difficult aspect of the ordering problem: how to decide the relative order of reference objects in different pockets. This is equivalent to assigning statistical weights to the different pockets once the ordering and hence the $\mathbf{p}$-distribution has been determined within each pocket. As detailed below, many attempts to solve the ordering problem have implicitly involved grouping the reference objects by the value of some physical parameter at their spacetime location [52-61], say by proper time $t$ (Figure 4, top panel), scale factor $a$ (Figure 4, middle panel) or density $\rho$ (roughly the black curves in top two panels).

In Section IV, we will review and discuss various ordering choices and their implications. This is a severe problem in the sense that the ordering strongly affects the testable prediction $f_{p}(\mathbf{p})$, yet we currently lack a compelling argument leading to a unique choice. As detailed below, it even affects the sense in which we should think of inflation as eternal. This embarrassing problem is clearly one of physics rather than philosophy, since its resolution affects the testable inflationary predictions for $f_{p}(\mathbf{p})$. On an optimistic note, the measure problem (how to compute probabilities) plagued both both statistical mechanics and quantum physics early on, so there is real hope that inflation too can overcome its birth pains and become a testable theory whose probability predictions are unique.

basin, but a basin can correspond to multiple pockets, either because of initial conditions (in Figure 4, imagine, say, an initial inflaton field $\phi(\mathbf{r})=\phi_{\text {peak }}+\epsilon \sin x$ that is draped over the maximum at $\phi_{\text {peak }}$ multiple times) or because of quantum diffusion (which can drape the inflaton field repeatedly over the maximum even starting with the simple initial condition $\phi(\mathbf{r})=\varepsilon x$ where the field crosses the maximum only once) [32]).

\section{Initial conditions and predictions}

Let us cast the above issues in mathematical form, including the initial conditions $\boldsymbol{\Phi}$ which are the inflaton field and its first derivative early on:

$$
\Phi \equiv\left(\begin{array}{c}
\phi \\
\dot{\phi}
\end{array}\right) .
$$

Given any particular initial conditions $\boldsymbol{\Phi}$ and inflaton potential $V(\phi)$, the standard inflation formalism allows us to compute the probability distribution $f_{p}(\mathbf{p} ; \boldsymbol{\Phi}, V)$ for the parameters $\mathbf{p}$. If quantum diffusion is negligible, then the parameters are uniquely determined by $\boldsymbol{\Phi}$ and $V$ and we have simply

$$
f_{p}(\mathbf{p} ; \mathbf{\Phi}, V) \approx \delta[\mathbf{p}-\mathbf{p}(\mathbf{\Phi}, V)],
$$

where $\mathbf{p}(\boldsymbol{\Phi}, V)$ is computed as in Section II or by going beyond the slow-roll approximation if necessary [11]. When quantum diffusion is important, a given starting point $\boldsymbol{\Phi}$ no longer gives a unique classical time-evolution $\phi(t)$ and so the probability distribution $f_{p}(\mathbf{p} ; \boldsymbol{\Phi}, V)$ widens — for this case, $f_{p}(\mathbf{p} ; \boldsymbol{\Phi}, V)$ can be evaluated either by solving a stochastic ordinary differential equation or, equivalently, by integrating a Fokker-Planck equation $[8,53,62,103-106]$. As will be discussed in Section IV, there are even some choices of measure where attractor dynamics $[8,53,62,103-106]$ causes $f_{p}(\mathbf{p} ; \boldsymbol{\Phi}, V)$ to be completely independent of the initial conditions $\boldsymbol{\Phi}$.

Quite generally, we can now write the theoretical prediction for the parameter probability distribution as

$$
f_{p}(\mathbf{p})=w(\mathbf{p})\left\langle\left\langle f_{p}(\mathbf{p} ; \boldsymbol{\Phi}, V)\right\rangle_{\boldsymbol{\Phi}}\right\rangle_{V},
$$

where $w(\mathbf{p})$ is a weight function reflecting the choice and ordering of reference objects. Here \langle\rangle$_{\boldsymbol{\Phi}}$ denotes averaging over initial conditions $\boldsymbol{\Phi}$, to cover cases where the theory predicts a distribution of initial conditions rather than some particular initial conditions. Analogously, \langle\rangle$_{V}$ denotes averaging over inflaton potentials $V$ to cover cases where this is necessary - on could, for instance, envision theories predicting a quantum superposition of many different effective inflaton potentials corresponding to different false vacua of the underlying theory.

For notational convenience, we augment our parameter vector from equation (1) to

$$
\mathbf{p} \equiv\left(\Omega_{\mathrm{tot}}, \rho_{\Lambda}, w, Q, n_{s}, \alpha, r, n_{t}, N_{\mathrm{tot}}\right),
$$

making it include also $N_{\text {tot }}$, the total number of e-foldings during inflation. Although, unlike the other parameters, $N_{\text {tot }}$ is not directly observable, it enters in many interesting choices of the weight function $w(\mathbf{p})$. In Section VI we will explore a variety of weight functions $w(\mathbf{p})$ including factors such as $e^{3 N_{\text {tot }}}$ (weighting by the thermalized volume produced), $\theta\left(N_{\text {tot }}-55\right)$ (a Heaviside step function giving weight only to regions inflating by at least 55 e-foldings, a requirement linked to galaxy formation), 
$f^{\text {halo }}\left(Q, \rho_{\Lambda}, \Omega_{\text {tot }}\right)$ (the fraction of protons ending up in dark matter halos) and $f_{Q}(Q)$ (a factor related to galaxy formation and planetary stability).

We will find that the choice of measure makes a dramatic difference, and leads to one of three qualitatively different situations for the theoretical prediction $f_{p}(\mathbf{p})$ :

1. Predictions independent of initial conditions because of attractor dynamics.

2. Predictions independent of initial conditions because selection effects in $w(\mathbf{p})$ probe only tiny nonspecial part of initial distribution.

3. Predictions dependent on initial conditions, i.e., on pre-inflationary physics.

Case 1 is discussed in Section VII, with the conclusion that some implementations of it are observationally ruled out. Case 2 is discussed in Section V and is a multidimensional analogy of the classic example of anthropic constraints on $\rho_{\Lambda}[61,64,67-71]^{6}$. For some cases explored in Section V, we will find the predictions to be independent of the details of the inflaton potential $V(\phi)$ as well. Cases 1 and 2 are nice in the sense that we can make quantitative inflationary predictions from $V(\phi)$ alone, without having a theory of pre-inflationary physics. On the other hand, case 3 offers an opportunity to learn about preinflationary physics from cosmological observations of $\mathbf{p}$.

In summary, we have seen that the when computing inflationary predictions, the choice of measure discussed in this section has just as important observational consequences as the choice of inflaton potential discussed in Section II. Moreover, we found that the measure problem splits into three parts: one straightforward (chosing reference objects), one hard (chosing an ordering) and one perhaps irrelevant (chosing initial conditions).

\section{POSSIBLE SOLUTIONS TO THE ORDERING PROBLEM}

Above we saw that to make inflation a testable physical theory, the ordering problem must be solved. This is a wide open problem in dire need of further work - the purpose of this section is not to give a solution, merely to describe some possible approaches to solving it. We will compare their strengths and shortcomings in the remainder of the paper, first using numerical computations

\footnotetext{
${ }^{6}$ Weinberg's argument [68] went as follows: Since galaxies only form for $\left|\rho_{\Lambda}\right| \lesssim 10^{-123}$, all models predicting an a priori probability distribution $f\left(\rho_{\Lambda}\right)$ are equivalent as long as the characteristic width of the distribution is $\gg 10^{-123}$ and the value $\rho_{\Lambda}=0$ is not in any way special. The probability distribution for $\rho_{\Lambda}$ seen from a reference object (in this case a galaxy) can therefore be computed independently of the detailed shape of the initial condition distribution $f\left(\rho_{\Lambda}\right)$, simply treating $f\left(\rho_{\Lambda}\right)$ as constant.
}

in Section V and then using analytic calculations in Section VII. We will see that all orderings can be viewed as regularization techniques to deal with infinities, and that many are observationally ruled out.

A variety of solutions to the ordering problem have been discussed in the literature, albeit using different terminology. We will consider two broad classes of orderings:

1. Global orderings based on some time variable (say $t$ or $a)$.

2. Pocket-based orderings (ordering separately within each thermalized region (pocket), then averaging the pocket results with some weighting (say equally, by $\phi$-volume or by $\mathbf{r}$-volume).

\section{A. Global time-based orderings}

The first class of orderings foliates spacetime into a sequence of three-dimensional spatial hypersurfaces, each corresponding to a fixed "time", and computes the parameter probability distribution $f_{p}(\mathbf{p})$ separately on each hypersurface. We will see that this is in many cases equivalent to simply ordering the reference objects by increasing formation "time". The utility of this approach stems from two facts:

- In many cases, the ordering problem vanishes on each individual hypersurface. With time variables like $t$ or $a$, a finite initial volume will give a finite hypersurface volume for any fixed future time, so that all orderings of the (finitely many) reference objects give the exact same answer $f_{p}(\mathbf{p})$.

- In many cases, $f_{p}(\mathbf{p})$ converges to a well-defined distribution as this "time" approaches infinity, eliminating the need to select a particular hypersurface.

As detailed below, this first class of orderings is intimately linked to the infamous problem of chosing a time variable in the inflationary Fokker-Planck equation, and suffers from serious problems [48-50, 52-61].

For all cases where the total number of reference objects grows exponentially over time, any asymptotic $t \rightarrow$ $\infty$ distribution for reference objects at fixed time will be identical to the asymptotic distribution for reference objects formed at time $\leq t$, because older objects which formed at time $\ll t$ are exponentially underrepresented. The latter distribution is the one corresponding to simply ordering all reference objects by their formation time, so we will use the expressions " $t$-ordering" and " $t$-foliation" interchangeably.

\section{B. Pocket-based orderings}

In contrast, attempting to use density, temperature or expansion rate (or indeed any quantity which, unlike $t$ or 
$a$, is physically observable) as a time variable to foliate by leads to the second class of orderings - such "time variables" do not specify the ordering sufficiently for inflation to predict a unique parameter probability distribution, since there will typically be infinitely many reference objects at any fixed "time". Specifically, infinite numbers of reference objects at a given density, say, can typically be found in each of multiple thermalized pockets (the U-shaped regions in Figure 4, say) and neither the intrapocket ordering nor the inter-pocket weighting has been specified - we will discuss multiple options for both.

\section{Intra-pocket orderings}

For the former, i.e., within a given pocket, all orderings clearly give the same answer if the volume is finite. We will study the following two orderings for the infinitevolume case:

1. Empirical ordering: Order the reference objects by increasing physical distance from some given point r.

2. Volume-based ordering: Order the reference objects sampling each spatial region in proportion to its relative volume fraction, defined in terms of how much it has inflated.

The empirical ordering is equivalent to the procedure that an empirically minded observer at $\mathbf{r}$ would adopt: compute the parameter probability distribution by averaging in a spherical volume centered at $\mathbf{r}$ (on a hypersurface defined by a fixed density, say) and then let the sphere radius approach infinity. This procedure has been advocated by [72, 73], who applied it on the hypersurface corresponding to the end of inflation. It is easy to see that its predictions depend only on which pocket the point $\mathbf{r}$ lies in, not on its location within that pocket.

In contrast, a volume-based ordering would sample each spatial region with a frequency proportional to how much is has expanded since some early reference instant - the latter is challenging to define unambiguously, and currently there is no rigorously defined implementation of a volume-based ordering. These two orderings are distinguished by their answer to the question "are some volumes more infinite than others", to which they answer "no" and "yes", respectively. Consider, for example, the spatial region with $0 \lesssim x \lesssim 22$ in Figure 4 . This is a single basin of attraction with infinite thermalized volume both to the left and right side of the plane $x=14$, corresponding to the inflaton having rolled down to the minimum from the left and from the right, respectively. This predicts a cosmological parameter distribution of the form

$$
f_{p}(\mathbf{p})=p_{L} \delta\left(\mathbf{p}-\mathbf{p}_{L}\right)+\left(1-p_{L}\right) \delta\left(\mathbf{p}-\mathbf{p}_{R}\right),
$$

where $\mathbf{p}_{L}$ and $\mathbf{p}_{R}$ are the cosmological parameter vectors corresponding to $\phi$ rolling down from the left and right sides, respectively, and $p_{L}$ is the fraction of the reference objects in regions where $\phi$ rolled down from the left. The vectors $\mathbf{p}_{L}$ and $\mathbf{p}_{R}$ are determined by the potential $V(\phi)$ alone as per Section II, whereas the constant $p_{L}$ depends also on the measure. As long as both half-basins have infinite volume, the empirical ordering will predict $p_{L}=0.5$, whereas a volume-based ordering will generally not. For instance, if the reference instant corresponds to a given density, then volume-based ordering assigns a larger probability to the side where it takes more e-foldings to roll down. For the multi-field case where $\phi$ is a $d$-dimensional vector, $\mathbf{p}_{L}$ and $\mathbf{p}_{R}$ are generically replaced by a continuum of parameter vectors corresponding to the directions in $\phi$-space from which the inflaton rolled down, and equation (31) gets generalized to a weighted angular average in $\phi$-space.

In summary, both the empirical and volume-based intra-basin measures agree within any finite sub-volume (where they both weight by volume, i.e., reduce to standard Lebesgue measure), but differ in how to resolve the ambiguities associated with infinite volumes.

\section{Inter-pocket weightings}

Once an intra-pocket ordering has been prescribed within each pocket, the inter-pocket weighting must specify how reference objects from the different pockets are to be merged into a single ordered sequence. For instance, ordering objects $A_{1}, A_{2}, \ldots$ from pocket $\mathrm{A}$ and objects $B_{1}, B_{2}, \ldots$ from pocket $\mathrm{B}$ jointly as $A_{1}, B_{1}, A_{2}, B_{2}, \ldots$ corresponds to giving equal weight to the two pockets, whereas $A_{1}, A_{2}, B_{1}, A_{3}, A_{4}, B_{2}, A_{5}, A_{6}, B_{3} \ldots$ gives twice as much weight to pocket $\mathrm{A}$ as to pocket $\mathrm{B}$. There are at least five obvious candidates for the inter-pocket weighting:

1. Equal weighting for each basin of attraction (in $\phi$ space)

2. Equal weighting for each pocket (in physical space)

3. Weighting by relative $\phi$-volume

4. Weighting by relative thermalized physical volume

5. Weighting by relative number of reference objects

1 and 2 generally differ, since many disconnected pockets can have $\phi$ roll down into the same basin of attraction. An explicit definition of 4 is given in [74] for the special case of inflaton potentials with a single peak.

Since inflation typically produces infinitely many pockets, there is a potential subtlety related to pocket ordering within the weighted average. We define two pockets to be of the same type if they are observationally indistinguishable, i.e., give the same $f_{p}(\mathbf{p})$. If there are only finitely many types of pockets, the inter-pocket weighting is fully specified by giving the weight for each type. If there are infinitely many types, however, a naive 
weighted average over the types is ambiguous, since the order in which one adds the types (or pockets) matters. This is why, most generally, one needs to merge the reference objects from all pockets into a single ordered sequence as stipulated above.

\section{Symmetry arguments}

In the following sections, we will explore the predictions of various orderings listed above, and find that they are sufficiently different that it might be possible to determine observationally which one, if any, is the correct one. In the spirit of attempting to predict rather than postdict the correct answer, we note that there are three heuristic arguments favoring orderings where different pockets are treated symmetrically, i.e., receive equal weight.

The first argument is that, mathematically, two sets contain the same number of objects if there exists a oneto-one correspondence between them. Applying this criterion to the case of a one-dimensional inflaton potential, all countable infinities are equal and hence all infinite half-pockets should get equal weight, which corresponds to empirical intra-pocket weighting and equal inter-pocket weighting.

The second argument involves the "pothole paradox" that will be presented in Section VIIF, and also favors equal intra-pocket weighting.

The third argument involves the following Gedanken experiment. Consider two basins of attraction in a onedimensional inflaton potential $V(\phi)$, and let $\mathrm{A}, \mathrm{B}, \mathrm{C}$ and $\mathrm{D}$ denote four infinite spatial regions where $\phi$ has rolled down from the left into basin 1 , from the right into basin 1 , from the left into basin 2 and from the right into basin 2 , respectively. In the special case where $V(\phi)$ has identical shape in the left parts of the two basins, i.e., where there is some offset $\Delta \phi$ such that $V(\phi)=V(\phi+\Delta \phi)$ for all $\phi$ in the first half-basin, then one can argue that volumes $\mathrm{A}$ and $\mathrm{C}$ should receive equal statistical weight by symmetry. ${ }^{7}$ Since empirical intra-basin weighting equates the weight of $\mathrm{A}$ with $\mathrm{B}$ and the weight of $\mathrm{C}$ with $\mathrm{D}$, transitivity of equivalence implies that $\mathrm{B}$ and $\mathrm{D}$ have equal weight, and therefore that the two pockets $\mathrm{A}+\mathrm{B}$ and $\mathrm{C}+\mathrm{D}$ have equal weight even though they are causally disconnected. Since this argument applies regardless of the potential shape in half-basins $\mathrm{C}$ and $\mathrm{D}$, it suggests that all half-pockets and hence all pockets have equal weight. The assumed shape-equivalence of $\mathrm{A}$ and $\mathrm{C}$ can be eliminated from the argument if we consider a messy random potential like in Section $\mathrm{V}$ with infinitely many basins where for any given half-basin, there will be another one that approximates its shape arbitrarily

\footnotetext{
7 This argument fails for measures where quantum diffusion/tunneling from nearby basins is important.
}

closely. In summary, this suggests that empirical intrabasin weighting implies equal inter-pocket weighting.

We close this section by noting that the ordering problem is closely linked (but not equivalent) to the conditional probability calculation corresponding to conditioning on a class of reference object. For example, chosing our reference objects to be "protons at time $t$ " implies using the above-mentioned $t$-foliation. In contrast, chosing reference objects to be "protons at density $\rho$ " (or conditioned on $T, H$ or another observable) does not fully specify an ordering for the reasons given above, merely implying a pocket-based ordering.

\section{MONTE CARLO ANALYSIS}

Above we discussed how the cosmological parameter probability distribution $f_{p}(\mathbf{p})$ predicted by inflation depends on the inflaton potential $V(\phi)$ (Section II) and on the measure (Section III). We wish to keep our discussion from becoming overly abstract, so let us now compute the predictions for some concrete examples. We will then quantify the measure dependence for a wider class of orderings below in Section VII.

\section{A. Choice of potentials}

Since the inflaton $\phi$ and the potential $V$ have units of mass and density $\left(\right.$ mass $\left.^{4}\right)$, respectively, let us write the potential as

$$
V(\phi)=m_{v}{ }^{4} f\left(\frac{\phi}{m_{h}}\right),
$$

where $f$ is a dimensionless function with values and derivatives of order unity, and the characteristic energy scales of the horizontal and vertical axes in a plot of $V(\phi)$ are absorbed into the two constants $m_{h}$ and $m_{v}$. If $V(\phi)$ emerges from some fundamental physical theory without fine-tuning, one might naturally expect $m_{h} \sim m_{v}$, but we will also explore the more general case where the horizontal and vertical energy scales are different.

Ideally, we would wish to explore generic potentials whose shape $f$ is derived from some fundamental theory. Currently lacking this, we will instead explore potentials that are generic in the sense of being randomly generated. We will explore the case where $f$ is a onedimensional Gaussian random field with unit variance and power spectrum $P(q) \propto q^{\gamma} e^{-q^{2} / 2}$ (see Figure 2). In practice, we define

$$
f(x)=\frac{a_{0}}{\sqrt{2}}+\sum_{k=1}^{n} a_{k} \cos \left(\frac{k x}{\sqrt{n}}\right)+a_{-k} \sin \left(\frac{k x}{\sqrt{n}}\right),
$$

where the $(2 n+1)$ Fourier coefficients $a_{-n}, \ldots, a_{n}$ are independent real-valued Gaussian random variables with zero mean and variance

$$
\left\langle a_{k}^{2}\right\rangle=A \sum q^{\gamma} e^{-\frac{q^{2}}{2}}
$$


$q \equiv k / \sqrt{n}$ and the normalization constant $A$ is chosen so that $\left\langle f(x)^{2}\right\rangle=\frac{1}{2} \sum_{k=-n}^{n}\left\langle a_{k}^{2}\right\rangle=1$. We take $n=100$ and $\gamma=0$ as our baseline, but explore a variety of other choices below. We will find that the resulting parameter probability distribution $f_{p}(\mathbf{p})$ is rather insensitive to the detailed shape of $f$, but depends strongly on the energy scales $m_{h}$ and $m_{v}$. A key goal of this section is to identify which features of a complicated potential $V(\phi)$ are most important in determining the testable predictions $f_{p}(\mathbf{p})$.

\section{B. Choice of measures}

We will compute results for two different measures that we term Measure A and Measure B. Both weight all halfbasins roughly equally, the difference being that A counts only those producing an infinite volume. Measure A thus corresponds roughly to the empirical intra-pocket ordering and the equal weighting of basins. As described below, this also corresponds approximately to a uniform distribution over the initial conditions $(\phi, \dot{\phi})$, including the volume weighting factor $e^{3 N_{\text {tot }}}$ into the $w(\mathbf{p})$-factor of equation (29) for Measure A but excluding it for Measure B. Both of these two measures may be the wrong choice, but they are least free from the most egregious paradoxes and observational contradictions that afflict many other measures as discussed below in Section VII, and hence instructive to explore quantitatively.

Specifically, we perform the following numerical experiment:

1. Generate a random potential $V(\phi)$, start at $\phi=0$.

2. Go uphill until a maximum is reached.

3. If $V<0$ (Big Crunch imminent) or the slow-roll approximation (SRA) is invalid $(\epsilon>1$ or $|\eta|>1)$, count as an $N_{\text {tot }}=0$ failure and stop, otherwise evolve according to the SRA.

4. If $\phi$ gets stuck in an eternally inflating local minimum, count as a failure and stop (will receive zero weight since no reference objects produced - not even protons).

5. When the SRA breaks down (which defines $a_{\text {end }}$ ), compute p using equations (11)-(18), taking $a_{\text {exit }}$ to be 55 e-foldings earlier $\left(a_{\text {exit }}=e^{-55} a_{\text {end }}\right)$ and evaluating $\rho_{\Lambda}=V(\phi)$ at the subsequent local minimum of the potential.

6. Repeat to obtain a statistically large sample, say $10^{9}$ times.

7. Repeat the entire experiment for a range of energy scales $m_{h}$ and power spectrum shape parameters $\gamma$.

Step 2 is optional: we include it for Measure A but not for Measure B. We are thus simulating "shotgun inflation" in the sense that we spray starting points randomly across the potential surface (Measure B) or at random maxima
(Measure A). In Section VI, we will use either galaxies or objects in galaxies as reference objects, so the weight function $w(\mathbf{p})$ from equation (29) will eliminate all cases where there is too much dark energy $\left(\left|\rho_{\Lambda}\right| \gg 10^{-112} Q^{3}\right)$ for galaxies to form or where black holes prevent the formation of reference objects (for $N_{\text {tot }} \lesssim 55$ as per Section VIC 2). As detailed in Section VII, Measure A will automatically give $N_{\text {tot }}=\infty$, whereas Measure B will give $N_{\text {tot }}=\infty$ only if the maximum above the starting point satisfies the SRA, i.e., has $\eta>-1$. This $N_{\text {tot }^{-}}$ cutoff thus affects only Measure B.

The two measures defined by this prescription were chosen to maximize computational efficiency, not to be maximally simple to interpret. However, we will now see that, modulo some weight factors or order unity, they correspond to weighting all half-basins equally and also to uniform initial conditions.

Since the statistical properties of our Gaussian random field $V(\phi)$ are translationally invariant, our choice to start at $\phi=0$ is equivalent to starting at a random $\phi$-value drawn from a uniform distribution. Apart from the numerical practicality that $n$ is finite so that $V$ is periodic, we can thus reinterpret our generation of many random potentials as simply trying many different starting points in the same infinitely extended potential. The probability that we roll down into any particular halfbasin is therefore proportional to its length for Measure A and to the length of the sub-region giving $N_{\text {tot }}>55$ for Measure B. The length of a half-basin (the distance between a maximum and a minimum of $V$ ) is of order $m_{h}$ with random variations of order unity, so the different half-basins get weighted roughly equally.

The parameter $\rho_{\Lambda}$ of course depends only on which basin we roll down into, since it is the height of its minimum. Since the potential is one-dimensional, the parameters $\left(Q, n_{s}, \alpha, r, n_{t}\right)$ depend only on the half-basin (whether we roll down from the left or from the right), so the only parameters affected by where in the basin we start are $\Omega_{\text {tot }}$ and $N_{\text {tot }}$ - for Measure A, where we start at a maximum, they are $\Omega_{\text {tot }}=1$ and $N_{\text {tot }}=\infty$.

Finally, let us discuss the effect of requiring the slowroll approximation (SRA) to hold. When instead using the exact inflationary dynamics of equation (A14), we find that almost all cases giving substantial amounts of inflation do so while the slow-roll approximation (SRA) is valid. Because our $w(\mathbf{p})$ gives negligible weight to cases with $\lesssim 55$ e-foldings (black holes prevent the formation of reference objects as per Section VI C 2), our statistics will be dominated by trajectories $\phi(t)$ involving at least one slow-roll period. Our prescription counts those starting with slow-roll and discards those where the SRA becomes valid only later on (for instance, a large $\dot{\phi}$ can be finely tuned to just barely make $\phi$ roll up a hill, so that the SRA becomes valid near the top). If $\epsilon<1$ and $|\eta|<1$ at a given position $\phi$, then the well-known SRA attractor behavior [11] will soon drive the derivative $\dot{\phi}$ to the SRA value $\dot{\phi} \approx-\bar{m} V^{\prime}(\phi) \sqrt{3 / V(\phi)}$ if it starts out in the slowroll range $|\dot{\phi}| \lesssim \sqrt{2 V(\phi)}$. If we began with random and 


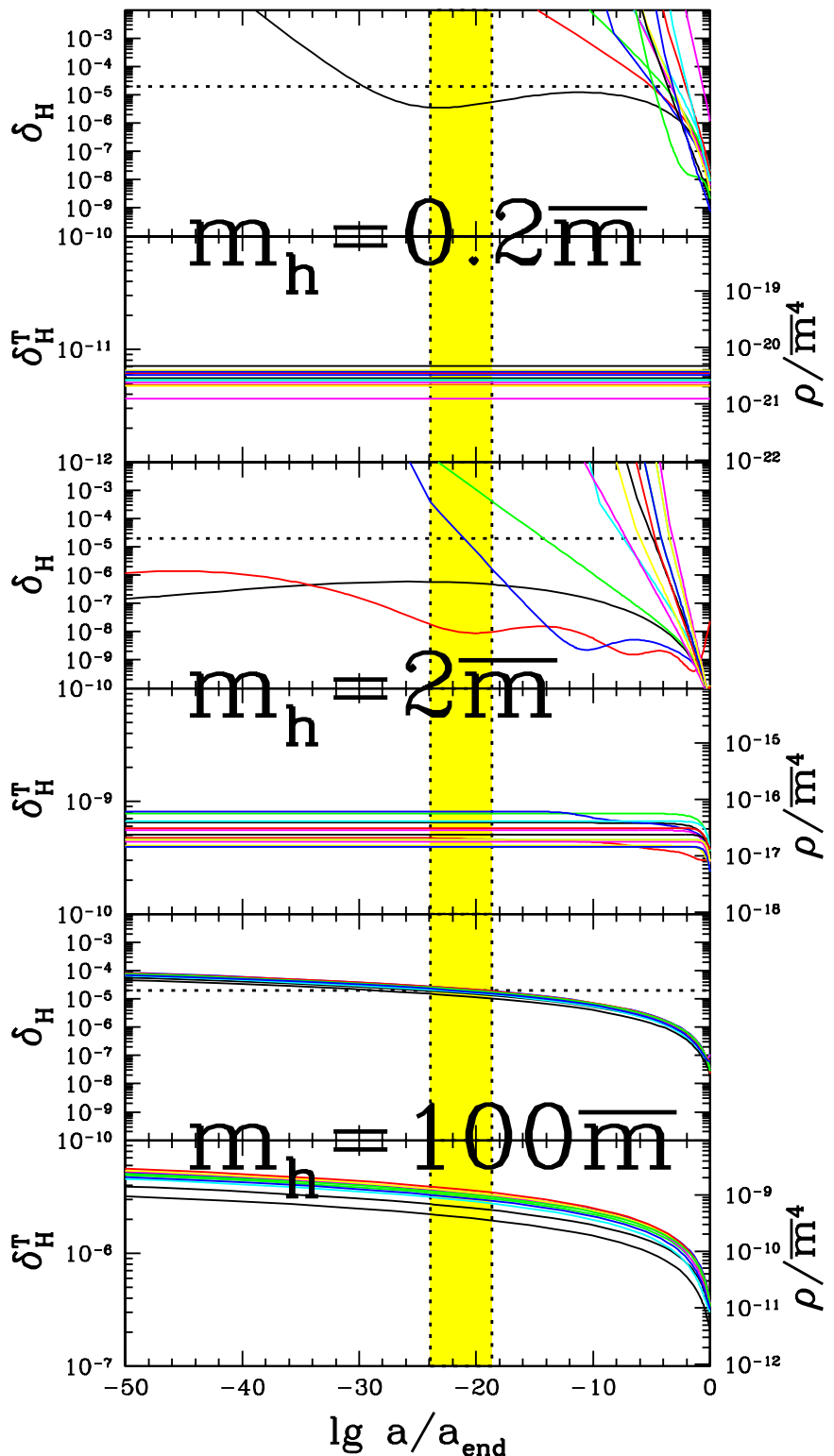

FIG. 5: The cosmic density history $\rho(a)$ is shown in the three panels with labeling on the right side for a handful of simulations with Measure $\mathrm{A}$ and inflation mass scales $\left(m_{h}, m_{v}\right)=\left(0.2 \bar{m}, 10^{-6} \bar{m}\right)$, $(2 \bar{m}, 0.0004 \bar{m})$ and $(100 \bar{m}, 0.02 \bar{m})$. The corresponding scalar and tensor power spectra are shown with labeling on the left side. The shaded vertical band indicates the range 43-55 e-foldings from the end of inflation where there is current hope to measure these primordial fluctuations [75, 76].

uniformly distributed initial conditions $\boldsymbol{\Phi}=(\phi, \dot{\phi})$, the fraction of the models surviving the $\dot{\phi}$ cut would thus be $\propto V^{1 / 2}$, so that the $\dot{\phi}$-cut is equivalent to starting with the SRA value of $\phi$ and weighting the initial $\phi$ distribution by $V(\phi)^{1 / 2}$. We find that $V^{1 / 2}$ does not vary strongly between successfully inflating models, so our prescription is roughly equivalent to simply starting with uniformly random initial conditions $\boldsymbol{\Phi}$.

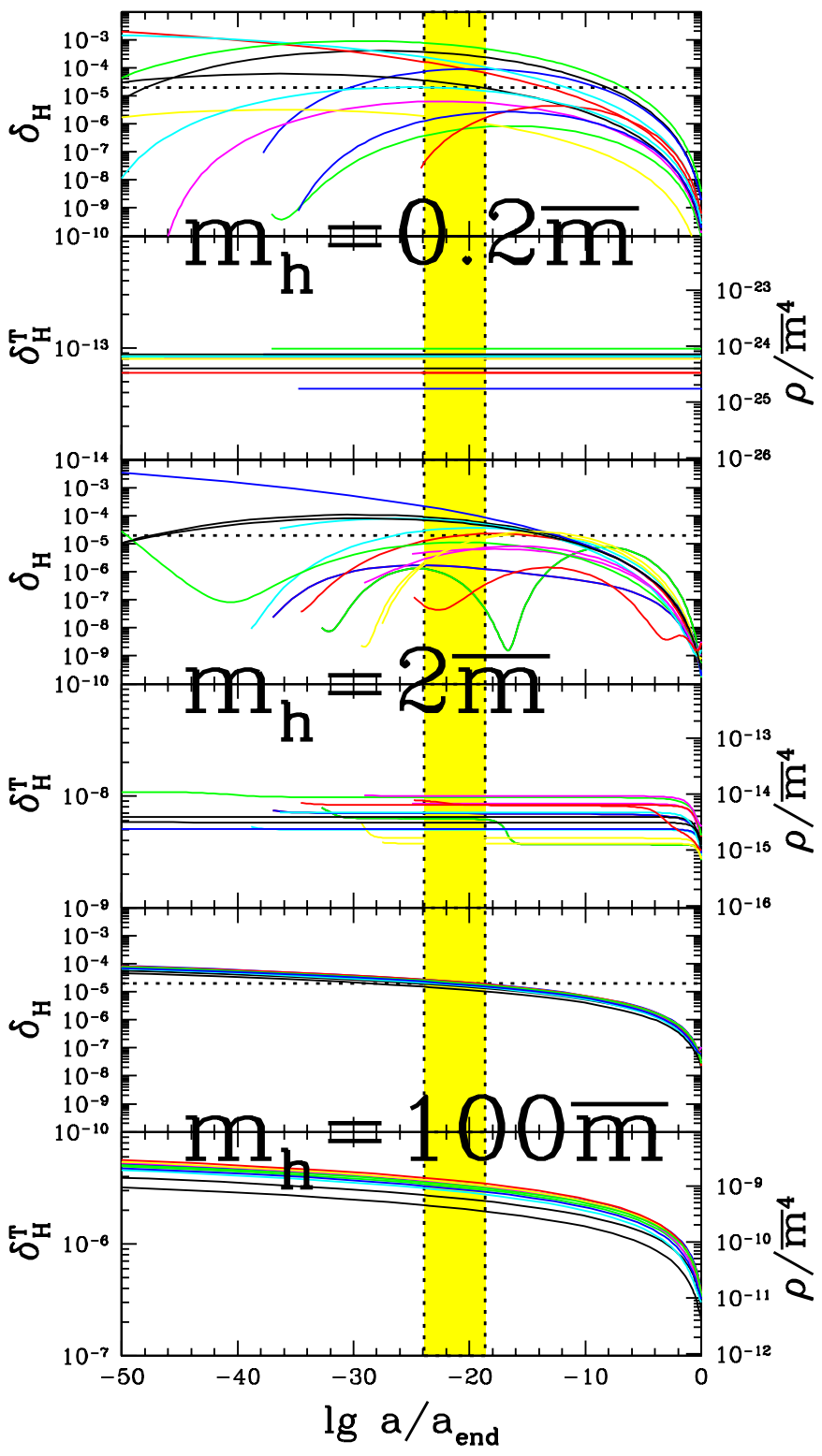

FIG. 6: Same as Figure 5, but for Measure B, which includes thermalized regions with finite volume.

For computational efficiency, we ignore quantum diffusion in our calculations. We will discuss the effects of diffusion in Section VG and find that they are unimportant for our qualitative conclusions. As discussed in Section VII, diffusion can, with some measures, drastically alter the relative weighting between different basins. Once we have specified the inter-pocket weighting, however, the only observable effect that diffusion can have is to modify the evolution during the last 55 or so e-foldings - this is important only for models with $Q \gtrsim 1$ and is completely irrelevant for models with $Q \sim 10^{-5}$. 


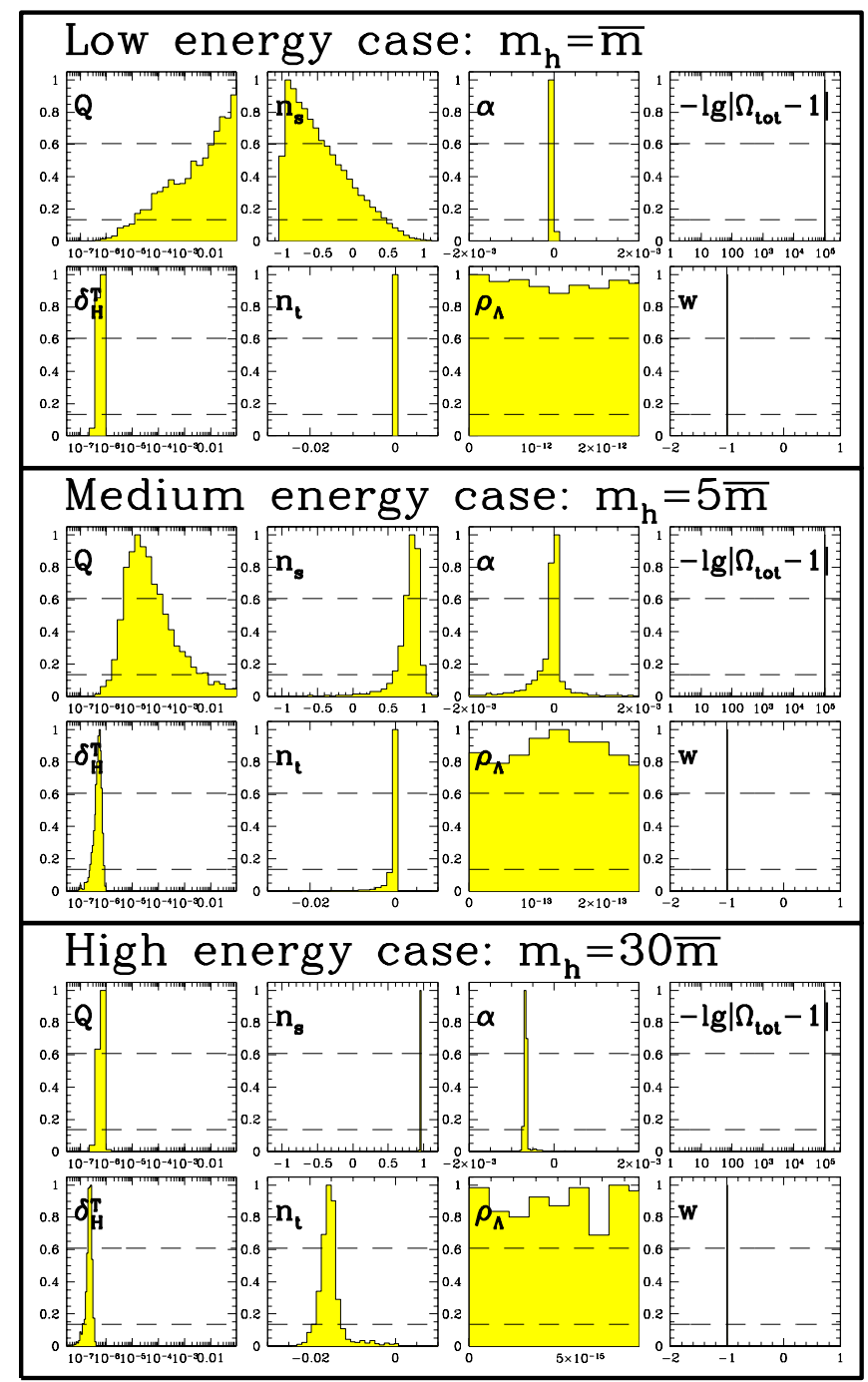

FIG. 7: The cosmological parameter distributions predicted by three inflation models with $m_{v} \sim 0.007 m_{v}$ and Measure A.

\section{Basic results}

Figures 5 and 6 shows the resulting $\rho(a)$-curves for a small sample of models for the two measures. From these curves, the cosmological functions $\delta_{H}(k)$ and $\delta_{H}^{T}(k)$ are readily obtained using equations (6) and (7) - these two figures show examples. Our cosmological parameters $\mathbf{p}$ are computed from the $\rho(a)$-curves using equations (11)(18), giving histograms such as those shown in Figures 7 and 8. Let us now explore how these results depend on the inflation mass scales $m_{h}$ and $m_{v}$ and on the parameter $\gamma$ controlling the power spectrum of the inflaton potential.

The $m_{v}$-dependence is readily computed analytically. Since quantum diffusion is negligible for the cases we consider, the vertical inflation scale $m_{v}$ affects none of the parameters except the overall amplitudes $Q$ and $Q_{t}=r Q$, which both scale as $m_{v}^{2}$ (the $\rho_{\Lambda}$-distribution is affected only indirectly via $Q$, as will become clear in Sec-

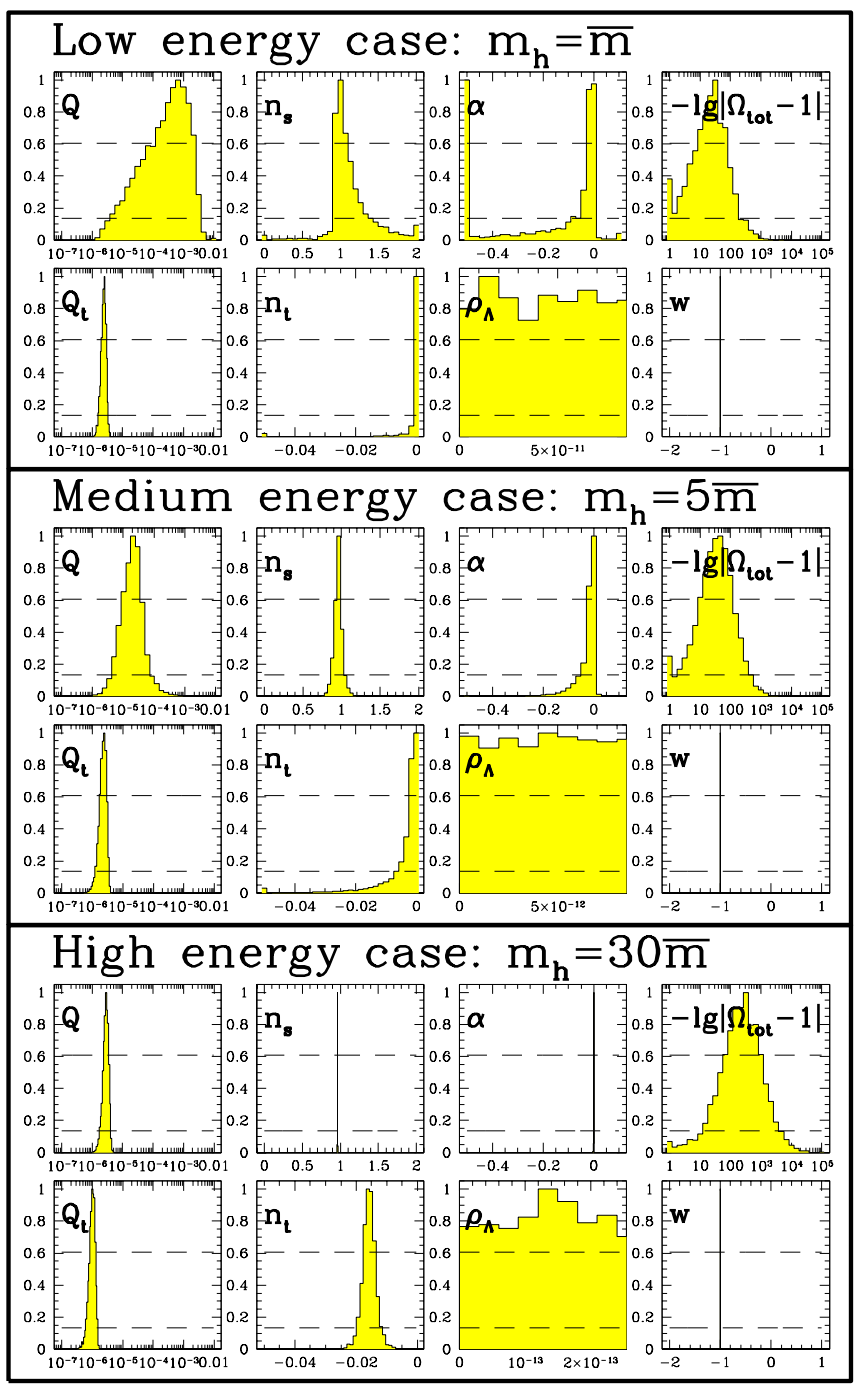

FIG. 8: The cosmological parameter distributions predicted by three inflation models with $m_{v} \sim 0.007 m_{v}$ and Measure B.

tion VI). The effect of changing the horizontal scale $m_{h}$ (the scale on which the inflaton potential wiggles), however, needs to be computed numerically, and is complex and interesting for most parameters. We compute the parameter distribution $f_{p}(\mathbf{p})$ for the values $m_{h} / \bar{m}=0.2$, $0.5,1,2,3,5,7,10,20,30,100$ and 1000 , and the results are summarized in Figures 9 and 10. For our baseline calculations, we take $\gamma=0$. We repeat the entire grid of calculations for $\gamma=2$ and $\gamma=4$ and find that this has little effect on the results - the analytic results below will clarify why.

We find that there are three regimes of horizontal mass scale giving qualitatively different behavior: low energy $m_{h} \ll \bar{m}$, intermediate energy $m_{h} \sim \bar{m}$ and high energy $m_{h} \gg \bar{m}$. We will now discuss these three regimes in turn. 


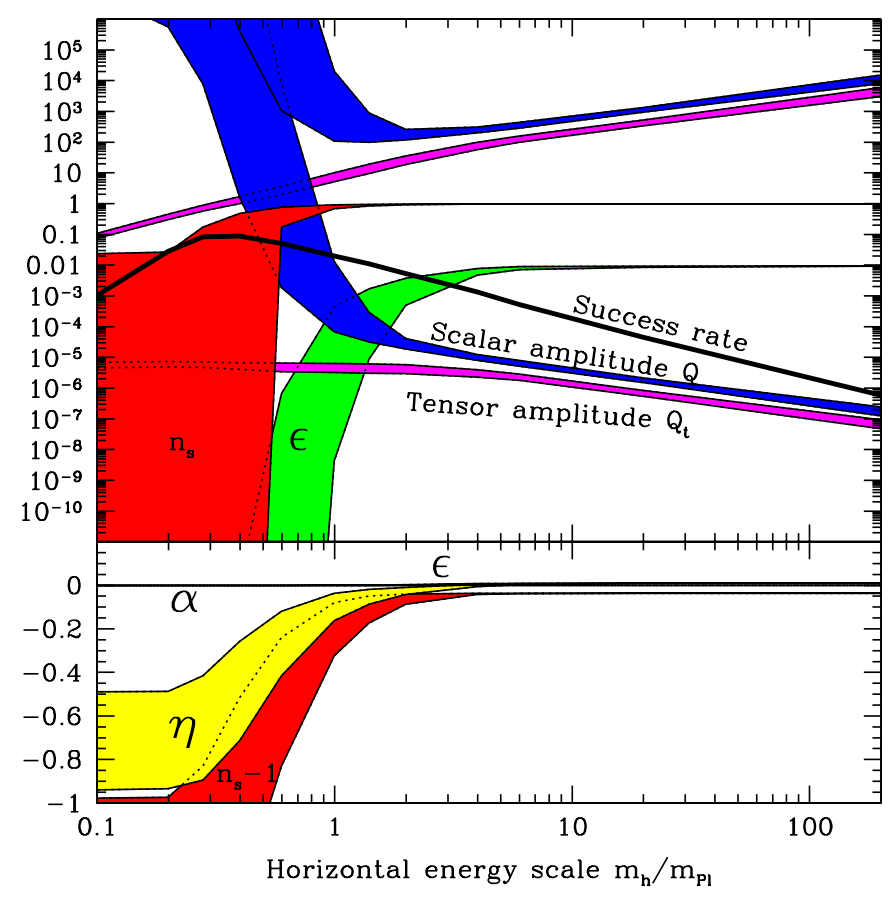

FIG. 9: Each shaded band shows the central $68 \%$ of a parameter probability distribution (such as those in Figure 7) as a function of horizontal inflation mass scale $m_{h}$, for Measure A. All labeled bands have the vertical scale fixed at $m_{v}=0.007 \bar{m}$. The second pair of bands for $Q$ and $Q_{t}$ (unlabeled, above labeled bands with same colors/shades) have $m_{v}$ varying so that $m_{v}=m_{h}$.

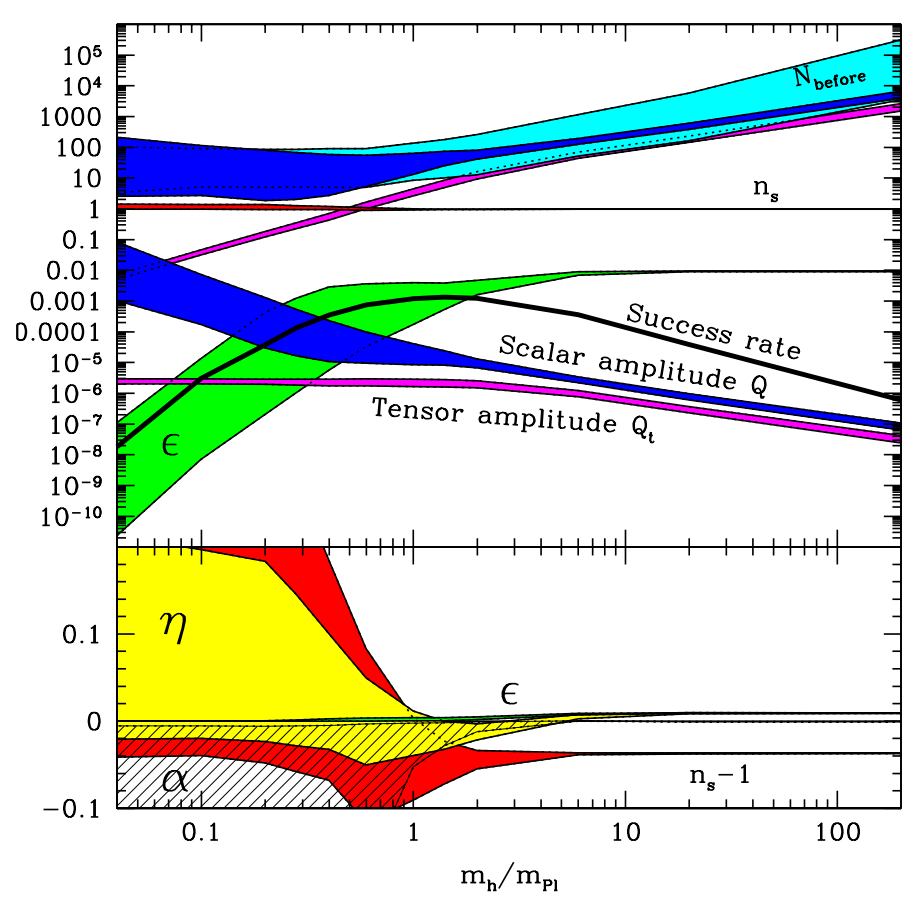

FIG. 10: Same as Figure 9, but for Measure B. which includes thermalized regions with finite volume.

\section{The high energy limit $m_{h} \gg \bar{m}$}

Although we lack a theory of quantum gravity, it is commonly assumed that quantum gravity corrections will make contributions to the inflaton potential in the form of a power series in $\left(\phi / m_{\mathrm{Pl}}\right)$ with coefficients of order unity, thus causing $V(\phi)$ to vary substantially when on a scale $\Delta \phi \sim m_{\mathrm{Pl}}$. In other words, one assumes that quantum gravity predicts $m_{h} \lesssim m_{\mathrm{Pl}} \sim 5 \bar{m}$, rendering the high-energy regime $m_{h} \gg \bar{m}$ physically unnatural and poorly motivated. This classic argument [8-15] is also the standard objection to large-field inflation models like $V(\phi) \propto \phi^{2}$, which require this sort of extreme flatness to agree with observation - see [77] for a counterarguments and discussion of these issues.

These issues aside, what are the testable predictions in the $m_{h} \gg \bar{m}$ limit? Figures 9 and 10 reveal an interesting limiting behavior: when $m_{h}$ increases far above the Planck scale, the predictions for $n_{s}, r$ and $\alpha$ become very sharp, with almost no random scatter, independently of whether Measure A or Measure B is used. This behavior can be traced back to Figures 5 and 6 , illustrating that as $m_{h} \rightarrow \infty$, all the $\rho(a)$-curves have the same shape, differing merely in amplitude. The slow-roll parameters $\epsilon$, $\eta$ and $\xi_{2}$, which depend only on the shape of $\rho(a)$, therefore approach constants and the corresponding probability distributions for $r, n_{t}, n_{s}$ and $\alpha$ in Figures 7 and 8 approach Dirac $\delta$-functions as $m_{h} \rightarrow \infty$.

The reason for this limiting behavior is easy to understand. Increasing $m_{h}$ makes it easier to satisfy the slowroll conditions $\epsilon \ll 1$ and $|\eta| \ll 1$, since equation (32) implies that

$$
\begin{aligned}
& \epsilon=\frac{1}{2}\left(\frac{\bar{m} V^{\prime}}{V}\right)^{2}=\frac{1}{2}\left(\frac{\bar{m}}{m_{h}}\right)^{2}\left(\frac{f^{\prime}}{f}\right)^{2} \sim\left(\frac{\bar{m}}{m_{h}}\right)^{2} \\
& \eta=\frac{\bar{m}^{2} V^{\prime \prime}}{V}=\left(\frac{\bar{m}}{m_{h}}\right)^{2} \frac{f^{\prime \prime}}{f} \sim\left(\frac{\bar{m}}{m_{h}}\right)^{2}
\end{aligned}
$$

at generic points $\phi$, so that as $m_{h} \rightarrow \infty$, inflation occurs at almost all $\phi$-values where $V(\phi)>0$. If inflation ever ends, it does so because $f$ drops very close to zero, either because of $\epsilon$ when $f=\left(m_{h} / \bar{m}\right)\left|f^{\prime}\right| / 2$ or because of $\eta$ when $f=\left(m_{h} / \bar{m}\right)^{2}\left|f^{\prime \prime}\right|$. Recall that the dimensionless inflaton potential $f$ is a function whose values and derivatives are generically of order unity. In either of the two cases, our $m_{h} \gg \bar{m}$ limit therefore implies that $0<f \ll 1$ when inflation ends, so that the observational consequences depend only on the behavior of $f$ over a tiny range very close to zero. Since this range is much smaller than the natural scale on which $f$ varies, the only aspects of $f$ that have any observational consequences are the first terms of its Taylor expansion in this range.

The most likely behavior in the region where $f \approx 0$ is that $f$ is roughly a straight line with slope of order unity. For most of our Monte Carlo simulations, inflation is therefore ended by $\epsilon=1$ when $f \sim \bar{m} / m_{h}$, after which the inflaton fast-rolls [6] down to negative values 
of the potential and space promptly recollapses without producing any galaxies or other reference objects.

Although these are the most common cases, they are given zero statistical weight by the $w(\mathbf{p})$-factor in equation (29) and hence do not contribute to the predicted parameter probability distribution $f_{p}(\mathbf{p})$. The only cases that produce galaxies are those where $f^{\prime}$ is unusually small in this region, so that $f$ flattens out and takes a local minimum where $\rho_{\Lambda}=V(\phi)=m_{v}{ }^{4} f\left(\phi / m_{h}\right)$ is close enough to zero to produce galaxies. Since this constraint is roughly $\left|\rho_{\Lambda}\right| \gg 10^{-112} Q^{3}$ (see Section VI), the only segments of a potential $V(\phi)$ that contribute to the parameter probability distribution are those whose minimum $V\left(\phi_{\text {stop }}\right)$ is much closer to zero than the $V$ value where inflation ends, in other words those segments that are well-approximated by a parabola $V(\phi) \propto \phi^{2}$. For this well-studied case $[11,32]$, we have $\epsilon=\eta=$ $2 \bar{m}^{2} /\left(\phi-\phi_{\text {stop }}\right)^{2}$, which gives $\phi_{\text {end }}=\phi_{\text {stop }} \pm \sqrt{2} \bar{m}$ and the following cosmological functions and parameters (see Appendix B and, e.g., [11]):

$$
\begin{aligned}
\rho(a) & =\rho\left(a_{\text {end }}\right)(1+2 N), \\
Q & =\frac{2 N+1}{\sqrt{75} \pi} Q_{*} \approx 4 Q_{*}, \\
Q_{t} & =\frac{4}{\sqrt{75} \pi} \sqrt{2 N+1} Q_{*} \approx 1.5 Q_{*} \\
n_{s} & =1-\frac{4}{2 N+1} \approx 1-\frac{2}{N} \approx 0.963, \\
\alpha & =-\frac{8}{(2 N+1)^{2}} \approx-\frac{2}{N^{2}} \approx-0.0006, \\
r & =\frac{16}{2 N+1} \approx \frac{8}{N} \approx 0.15 \\
n_{t} & =-\frac{2}{2 N+1} \approx-\frac{1}{N} \approx 0.02
\end{aligned}
$$

where $N \equiv a_{\text {end }} / a$ as usual, $Q_{*} \equiv \frac{m_{v}{ }^{2}}{2 \bar{m} m_{h}} \sqrt{f_{\text {min }}^{\prime \prime}}$ and $f_{\min }^{\prime \prime} \sim 1$ is the second derivative of the dimensionless inflaton potential $f$ at the local minimum into which it rolls down. The number of e-foldings $N$ corresponding to our current horizon volume is given by [36]

$$
N \approx 63.3-\frac{1}{12} \ln \frac{\rho_{\mathrm{end}}}{\rho_{\mathrm{reh}}},
$$

where the last term is $0,4,11$ and 15 for reheating instantaneously, at $\lesssim 10^{11} \mathrm{GeV}$ (to avoid overproduction of gravitinos in SUSY models), at the electroweak scale $100 \mathrm{GeV}$ and at the BBN scale $1 \mathrm{MeV}$ (at lower reheat energies, no protons are produced — but see [78]). We have used $N=55$ in all our plots. Finally, we expect the observed $\Omega_{\text {tot }} \sim 10^{-5}$ as $m_{h} \rightarrow \infty$, since the decreased slow-roll speed increases the total number of e-foldings needed to roll down from a given starting point $\phi$, giving $N_{\text {before }} \equiv \ln \left(a_{\text {start }} / a_{\text {exit }}\right) \rightarrow \infty$ as $m_{h} \rightarrow \infty$.

The above analytic arguments are confirmed by our numerical calculations. Figures 5 and 6 (bottom) show that the density histories $\rho(a)$ have the shape predicted by equation (37) - the scatter in amplitudes is due to the fact that $\rho_{\text {end }} \propto Q_{*}^{2} \propto f_{\text {min }}^{\prime \prime}$, and this second derivative of order unity differs from one minimum to another. Figures 9 and 10 show that the dispersions in $n_{s}, r, n_{t}, \alpha, \epsilon$, $\eta$ and $\xi_{2}$ drop towards zero as $m_{h}$ increases, and that the constant values they approach are precisely those given by equations (40)-(43), i.e., $\left(n_{s}, r, n_{t}, \alpha, \epsilon, \eta, \xi_{2}\right) \approx$ $(0.963,0.15,-0.02,-0.0006,0.009,0.009,0)$.

Also, Figure 10 shows that the success rate drops $\propto m_{h}{ }^{-2}$ as $m_{h} \rightarrow \infty$, as predicted by our above discussion: inflation ends with $\eta \sim 1$ when $f=f_{\text {end }} \sim$ $\left(m_{h} / \bar{m}\right)^{2} f^{\prime \prime} \sim\left(m_{h} / \bar{m}\right)^{2} \ll 1$ according to equation $(36)$, and the probability that the next minimum lies between this tiny positive value and zero is thus proportional to $f_{\text {end }} \underset{\sim}{\sim} m_{h}{ }^{-2}$.

Equation (38) shows that reproducing the observed fluctuation level $Q \approx 2 \times 10^{-5}$ requires $m_{h} \sim$ $0.003 \sqrt{\bar{m} m_{v}}$, so in addition to the possible quantum gravity problems with having $m_{h} \gg 1$, these models also require tuning in the sense that $m_{v} \ll m_{h}$ (they are far from the band marked "natural" in Figure 11).

In summary, the parabolic inflaton potential $V(\phi) \propto$ $\phi^{2}$ has been widely studied in the literature, and is a classic example giving eternal inflation with an unbounded potential [32]. It is also the only integer power law potential that is still consistent with observational data now that the $\phi^{4}$ model is firmly ruled out [3]. In this subsection, we have seen that the predictions of this particular model hold also for a much broader class of models. Although our Monte Carlo calculations were only for the case of Gaussian random field potentials, our analytic arguments above clearly hold for any generic dimensionless inflaton potential $f$, and also independently of the initial conditions. Specifically, in the high-energy limit $m_{h} \gg \bar{m}$, any messy and complicated inflaton potential predicts the same values of $\left(n_{s}, r, n_{t}, \alpha, \epsilon, \eta, \xi_{2}\right)$ as the simple $V(\phi) \propto \phi^{2}$ model as long as it is not fine-tuned to have its second derivative vanishing in a large fraction of its minima that $\phi$ can roll into, i.e., as long as generic minima look locally like parabolas. Of the eight cosmological parameters from equation (1), we thus obtain sharp predictions for $\left(\Omega_{\mathrm{tot}}, w, n_{s}, \alpha, r, n_{t}\right)$. In contrast, the probability distributions retain finite widths for $\rho_{\Lambda}$ and $Q$, which depend on $V(\phi)$ and $V^{\prime \prime}(\phi)$ at the minimum rolled down into. This means that in the $m_{h} \gg \bar{m}$ limit, the only aspect of the potential $V(\phi)$ that affects the cosmological parameter predictions $f_{p}(\mathbf{p})$ is the twodimensional distribution of $\left(V, V^{\prime \prime}\right)$ at its minima.

\section{E. The low energy limit $m_{h} \ll \bar{m}$}

\section{Motivation}

Although the above-mentioned limit $m_{h} \gg \bar{m}$ can match observational data, it has two theoretical blemishes as illustrated in Figure 11: quantum gravity correc- 


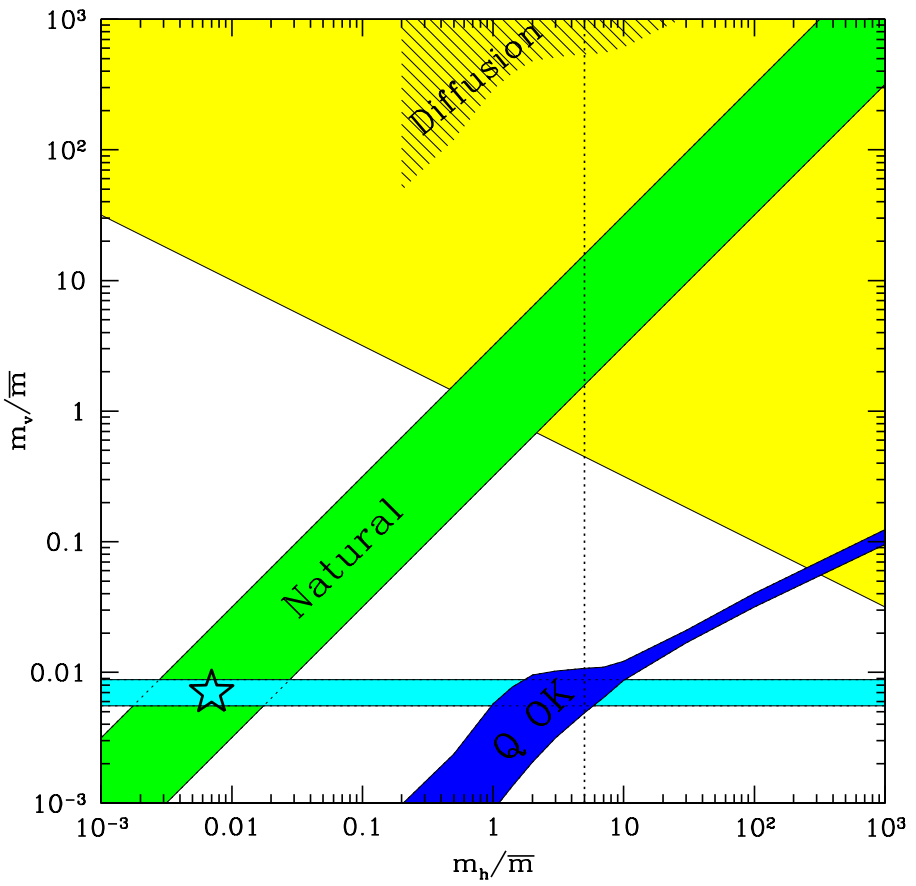

FIG. 11: The inflationary model space spanned by the energy scales $m_{h}$ and $m_{v}$ giving the horizontal and vertical units of the inflaton potential. Quantum gravity corrections may disfavor models with $m_{h} \gtrsim m_{\mathrm{Pl}} \approx 5 \bar{m}$ (to the right of the vertical line). The physically natural expectation is $m_{v} \sim m_{h}$ (green diagonal band), so one may hope to find a working model at the star, in the intersection of this band with the cyan horizontal band that gives the correct fluctuation amplitude $Q \sim 2 \times 10^{-5}$ when $m_{h} \sim m_{\mathrm{Pl}}$. We find that such hopes are dashed by the fact that $m_{v}$ must be lowered when $m_{h}$ is decreased (blue/dark grey band for Measure B), to offset the $Q$-increase caused by the preference for tiny $\epsilon$. One might naively expect quantum diffusion to be important in the yellow/light grey region, but the drop in $\langle\epsilon\rangle$ as $m_{h} \rightarrow 0$ and the drop in $\left\langle V\left(\phi_{\text {end }}\right)\right\rangle$ as $m_{h} \rightarrow \infty$ make diffusion important in the hatched region instead.

tions may render $m_{h} \gg \bar{m}$ unnatural, and the discrepancy $m_{h} \gg m_{v}$ between the two mass scales appears contrived. A natural expectation would be $m_{v} \sim m_{h} \sim m$ for some energy scale $m$ corresponding to the physics responsible for inflation, say the GUT scale or the string scale. $^{8}$

We find that for $m_{h} \sim m_{\mathrm{Pl}}$, the observed fluctuation amplitude $Q \sim 2 \times 10^{-5}$ is reproduced if $m_{h} \sim 0.007 \bar{m}$, which suggests exploring whether inflation with $m_{h}=$

\footnotetext{
8 In the context of some supersymmetric models, the "natural" expectation in Figure 11 is different and perhaps more compatible with what is observed. The reason is that in a supersymmetric theory, one naturally has two different scales: a high scale (say $\bar{m}$ ) in the ultraviolet and the SUSY breaking scale $M$. In many supersymmetric theories, the natural scales for the potential equation (32) of so-called moduli fields are $m_{v}=M$ and $m_{h}=\bar{m}$, typically with $m_{v} \ll m_{h}[79]$.
}

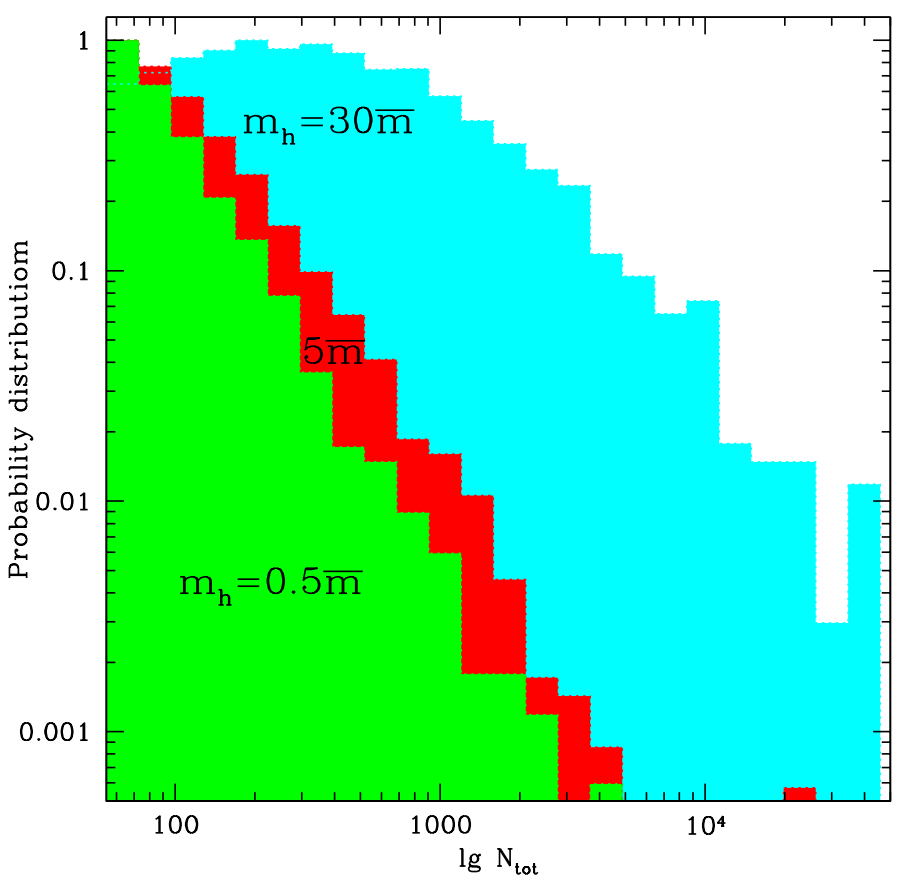

FIG. 12: The three histograms show the distributions of the total number of e=foldings $N_{\text {tot }}$ for high-energy $\left(m_{h}-30 \bar{m}\right.$, top) intermediate energy $\left(m_{h}=5 \bar{m}\right.$, middle $)$ and low-energy $\left(m_{h}=0.5 \bar{m}\right.$, bottom) examples with Measure B.

$m_{v} \sim 0.007 \bar{m}$ (five-pointed star in Figure 11) makes predictions in agreement with observation. We will see that this idea, which was one of the original motivations for the calculations in this paper, fails. However, we will find that it clears one of the most challenging hurdles and also gives predictions largely independent of the details of $V(\phi)$, so it will be interesting to see whether future work can resuscitate it in some modified form.

\section{The flatness constraint}

Figures 9 and 10 show that as we reduce $m_{h}$ from the Planck scale to lower values, the success rate drops. The reason for this is obvious from equations (35) and (36): $\epsilon$ and $|\eta|$ exceed unity at most $\phi$-values when $m_{h} \ll \bar{m}$, so that we obtain substantial inflation only when getting lucky and starting in a patch where $\epsilon$ and $|\eta|$ are anomalously small. The smaller $m_{h}$ is, the luckier we need to get and the lower the success rate will be. This wellknown fact that very wiggly potentials are less likely to support inflation was identified early on [80-84].

Given that inflation is intrinsically unlikely in this lowenergy limit, the observed flatness constraint $\left|\Omega_{\text {tot }}-1\right| \lesssim$ $0.02[1,2]$ becomes a challenging hurdle that can potentially rule out models with $m_{h} \ll \bar{m}$. If galaxy formation requires only $N_{\text {tot }} \gtrsim 55$ (see Section VI) and inflation is unlikely, is it not highly unlikely to get the additional 
e-foldings required to satisfy the observational flatness constraint, $N_{\text {tot }} \gtrsim 57$ ?

For Measure A, the answer is automatically no, since it automatically gives $N_{\text {tot }}=\infty$, so let us limit our discussion in this subsection to Measure B. Perhaps surprisingly, Figure 10 shows that the answer is no even for Measure B, with low energy inflation generally passing this observational test. In Figure 10, the number of additional non-required e-foldings $\left(N_{\text {before }}\right)$ is indeed seen to drop from $\sim 10^{5}$ to $\sim 10^{1}-10^{2}$ as $m_{h}$ is reduced from $10^{3} \bar{m}$ down to the Planck scale, but then more or less stops dropping as $m_{h}$ is further reduced. Figure 8 illustrates that the histogram for $\left|\Omega_{\text {tot }}-1\right| \sim e^{-2 N_{\text {before }}}$ is almost identical for the $m_{h}=5 \bar{m} \approx m_{\mathrm{Pl}}$ case and that with 5 times smaller $m_{h}$, typically giving values like $\Omega_{\mathrm{tot}}=1 \pm 10^{-30}$. Why is this? The basic reason is that, as seen in Figure 12, the $N_{\text {tot }}$-distribution for $m_{h} \ll m_{\mathrm{Pl}}$ is roughly a power law $f_{N}\left(N_{\text {tot }}\right) \sim N_{\text {tot }}^{p}$ with a rather modest slope $p \sim-3$ that steepens only slightly when $m_{h}$ is further decreased (the plotted distribution of $\lg N_{\text {tot }}$ thus has slope $N_{\text {tot }}^{p+1}$ ). The $m_{h}=0.2 \bar{m}$ case is not plotted in Figure 12, since it is virtually identical to the $m_{h}=0.5 \bar{m}$ case shown. This means that the probability that the total number of e-foldings $N_{\text {tot }}$ exceeds some given value $N_{*}$ satisfies $P\left(N_{\text {tot }}<N_{*}\right) \stackrel{\sim}{\sim} N_{*}^{p+1} \sim 1 / N_{*}^{2}$, so that the probability of satisfying the observed flatness constraint given that we are observing from a galaxy is

$$
P\left(N_{\text {tot }}>57 \mid N_{\text {tot }}>55\right) \sim\left(\frac{57}{55}\right)^{p+1} \sim 0.9 .
$$

In contrast, the flatness constraint would rule out $m_{h} \ll \bar{m}$ if the $N_{\text {tot-distribution were a much steeper }}$ power law or had an exponential cutoff, so let us un-

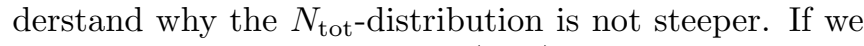
rewrite the slow-roll equation (A17) as

$$
d N \approx \frac{|d \phi|}{\bar{m}^{2}(\ln V)^{\prime}}=\frac{|d \phi|}{\bar{m} \sqrt{2 \epsilon}},
$$

we see that there are two different ways in which we can get lucky and obtain large $N_{\text {tot }}$ when faced with tiny $m_{h}$ : by rolling far (large $\Delta \phi$ ) or by being in a region where the potential is extremely flat $(\epsilon \ll 1)$. As we will now see, our results follow from the fact that the latter way of getting lucky is much more likely than the former. To get any inflation, we need $\epsilon<1$ and $|\eta|<1$ at our starting point, which according to Equations (35) and (35) requires $\left|(\ln f)^{\prime}\right| \lesssim m_{h} / \bar{m} \ll 1$ and $\left|f^{\prime \prime}\right| / f \lesssim\left(m_{h} / \bar{m}\right)^{2} \ll 1$. Since $f$ and its derivatives are by definition of order unity, the $\eta$-requirement is more stringent and will be satisfied for only about a fraction $\left(m_{h} / \bar{m}\right)^{2}$ of our simulations, showing that the success rate must drop at least as fast as $\left(m_{h} / \bar{m}\right)^{2}$ as $m_{h} \rightarrow 0$ (indeed, Figure 10 shows it dropping roughly as $m_{h}{ }^{6}$ ). This argument also predicts that almost all $m_{h} \ll \bar{m}$ models will have inflation terminated by $|\eta|$ exceeding unity while we still have $\epsilon \ll 1$, which is borne out by our simulations (for instance, out of our 1410065405 $m_{h}=0.5 \bar{m}$ simulations, every one of the 4260 that gave $N_{\text {tot }}>55$ were terminated by the $\eta$-constraint and none by the $\epsilon$-constraint). This is why the density histories look so extremely flat in Figure 6 (top panel). There is, however, an even stronger pressure towards tiny $\epsilon$. Since the slow-roll constraints must keep holding at all $\phi$-values that we roll through, we have to keep getting lucky with $\epsilon$ and $\eta$ over and over again as we roll along, and the probability of this happening drops faster than any power law in the rolled distance $\Delta \phi / m_{h}$ as higher and higher derivatives in the initial Taylor expansion become important. Figure 10 shows that empirically, by far the most likely way to get large numbers of e-foldings us therefore to roll very little, by having an anomalously small $\epsilon$ and hence a tiny roll distance $\Delta \phi$. Figure 10 shows that $\epsilon \sim\left(m_{h} / m\right)^{6}$ at the lowest energies probed, i.e., $(\ln V)^{\prime} \sim\left(m_{h} / m\right)^{3}$, to be contrasted with the expectation $(\ln V)^{\prime} \sim\left(m_{h} / m\right)^{-1}$ from equation (35). In summary, success requires getting lucky both with $\epsilon$ (which happens a fraction $\sim\left(m_{h} / m\right)^{4}$ of the time) and with $\eta$ (which happens a fraction $\sim\left(m_{h} / m\right)^{2}$ of the time), which together explains the empirically observed success rate $\epsilon \sim\left(m_{h} / m\right)^{6}$.

In conclusion, we can obtain large numbers of $e$ foldings with low-energy inflation by simply by getting lucky with tiny $\left|V^{\prime \prime}(\phi)\right|$ and minuscule $V^{\prime}(\phi)$ at the starting point $\phi$. Since zero is not a special value in the probability distributions for $V^{\prime}$ and $V^{\prime \prime}$ (as opposed to very large values, say, which may be exponentially unlikely), this is only polynomially unlikely.

\section{Predictions for the other 7 parameters}

Above we saw that despite their low success rate, $m_{h} \ll \bar{m}$ models are not ruled out by their predictions for the curvature parameter $\Omega_{\text {tot }}$. Let us now explore their predictions for the remaining 7 parameters. We will first focus on the case of Measure A, which is arguably the better motivated of the two since it weights by thermalized volume. We will then turn to Measure B, and find that its predictions and problems are qualitatively similar to those of Measure A.

Above we saw that the $m_{h} \gg \bar{m}$ limit simplified because the SRA was so easy to satisfy: the only properties of $V(\phi)$ that mattered were its minima, since the observable fluctuations where produced very near them. For the $m_{h} \gg \bar{m}$ limit, there is an analogous simplification: the SRA is now so hard to satisfy that the only properties of $V(\phi)$ that really matter are are the maxima, since the observable fluctuations are produced near them. For Measure A, where the thermalized pockets produced are infinite, we know that we began by rolling off a peak where $-1<\eta_{0} \leq 0$. Generic peaks have non-vanishing second derivative, i.e., $\eta_{0}<0$, and thus look locally like an upside-down parabola - for this well-know case, reviewed in Appendix B.2, the distance of $\phi$ from the peak grows $\propto a^{\eta_{0}}$, i.e., exponentially with the number of e- 
foldings. As shown in Appendix B.2, inflation around more general peaks is usually terminated when higher derivatives make $\eta$ exceed unity, and for the observable regime $N \sim 55$ gives the cosmological parameter predictions (see Appendix B)

$$
\begin{aligned}
\epsilon & \sim \frac{\eta_{0}^{2} m_{h}{ }^{6}}{\bar{m}^{6}} e^{2 \eta_{0} N} \approx 0, \\
\rho(a) & \approx V\left(\phi_{0}\right), \\
Q & =\frac{Q_{t}}{4 \sqrt{\epsilon}} \sim \frac{m_{v}{ }^{2} \bar{m}}{m_{h}{ }^{3} \eta_{0}} e^{-\eta_{0} N}, \\
Q_{t} & \approx \sqrt{\frac{8 V\left(\phi_{0}\right)}{75 \pi^{2} \bar{m}^{4}}} \sim \frac{m_{v}{ }^{2}}{\bar{m}^{2}}, \\
n_{s} & \approx 1+2 \eta_{0}, \\
\alpha & \approx-2 \xi_{2} \sim \eta_{0} e^{\eta_{0} N} \approx 0, \\
r & =16 \epsilon \approx 0, \\
n_{t} & =-2 \epsilon \approx 0, .
\end{aligned}
$$

Here the $\sim$ symbols indicate scatter of order unity in the relations stemming from $f$ and its derivatives. The most important scatter therefore comes not from this, but from the strong dependence on $\eta_{0}$ which, by the infinite-volume requirement, is a random variable in the range $(-1,0)$. Equation (47) shows that for $N \sim 55$, the four parameters $\epsilon, \alpha, r$ and $n_{t}$ will be exponentially small unless $\eta_{0} N \lesssim 1$, i.e., unless $-0.02 \lesssim \eta_{0}<0$. Since equation (36) shows that the curvature $\eta_{0}$ is much larger at generic extrema $(|\eta| \gg 1)$, we thus expect this exponential suppression of order $98 \%$ of the time ${ }^{9}$. Even for the remaining $\sim 2 \%$ of the models, these four parameters will be tiny, since equation (47) gives $\epsilon \lesssim N^{-2}\left(m_{h} / \bar{m}\right)^{6} \lesssim$ $10^{-9}$ for $m_{h}<0.1 \bar{m}$.

Figures 5, 7 and 9 show that these analytic predictions are borne out by our numerical calculations: for $m_{h} \ll$ $\bar{m}$, the parameters $\epsilon, \alpha, r$ and $n_{t}$ are all near zero and $n_{s}$ asymptotes to a broad distribution with range $-1<$ $n_{s}<1$. As suggested by equations (49) and (51), the distributions for $\ln Q$ and $n_{s}$ are seen to have the same shape up to a sign reversal, this common shape being simply that of the $\eta_{0}$-distribution since up to additive factors, $-\ln Q$ and $n_{s}$ equal $2 \eta_{0}$ and $N \eta_{0}$, respectively.

Whereas the predictions when $m_{h} \gg \bar{m}$ agreed well will observational data for a suitably tuned $m_{h}$-value, the predictions when $m_{h} \ll \bar{m}$ agree quite poorly with the observed spectral index $n_{s} \approx 0.98 \pm 0.02$, mostly

\footnotetext{
9 Specifically, this suggests that the $\eta_{0}$-distribution cannot have a characteristic scale on this comparatively tiny sub-interval $(-1,0)$, and therefore can be well approximated by a power law. The $\ln Q$-distribution is the convolution of the broad and featureless power-law distribution for $N \eta_{0}$ with a much narrower distribution of width $\Delta \ln Q \sim 1$ reflecting when exactly the $f$ factors end inflation, and will therefore have essentially the same power-law distribution as $N \eta_{0}$ over most of the range, i.e., be for all practical purposes determined by the distribution of peak curvatures $\eta_{0}$ alone.
}

being way too red. In addition, equation (49) shows that generic $\eta_{0}$-values predict $Q$-values that are exponentially large compared to the vertical energy scale $m_{v}$ : for the extreme case $\eta_{0}=1$, matching the observed value $Q \approx$ $2 \times 10^{-5}$ would require

$$
m_{v} \sim \sqrt{\frac{m_{h}^{3} Q}{\bar{m}}} e^{-N / 2} \sim 60 \mathrm{TeV} \times\left(\frac{m_{h}}{\bar{m}}\right)^{3} .
$$

These two potential problems are clearly linked, since they both correspond to $\eta_{0} \ll 0$. We will return to these interesting issues in Section VI, since they require a careful treatment of selection effects related to the $Q$ parameter.

Although our Monte Carlo calculations were only for the case of Gaussian random field potentials, our analytic arguments above clearly hold for any generic dimensionless inflaton potential $f$, and also independently of the initial conditions. Specifically, in the high-energy limit $m_{h} \gg \bar{m}$, any messy and complicated inflaton potential predicts negligible values of $\left(r, n_{t}, \alpha, \epsilon, \xi_{2}\right)$ as long as it is not fine-tuned to have its second derivative vanishing in a large fraction of the maxima with $\eta>-1$, i.e., as long as generic maxima look locally like upside-down parabolas (we cover the case of unbounded potentials in Section VG). Of the eight cosmological parameters from equation (1), we thus obtain sharp predictions for $\left(\Omega_{\mathrm{tot}}, w, \alpha, r, n_{t}\right)$. In contrast, the probability distributions retain finite widths for $n_{s}$ and $Q$ (which are highly correlated since they both depend on the peak curvature $\left.\eta_{0}\right)$ and for $\rho_{\Lambda}$ which depends on $V(\phi)$ at the subsequent minimum. In Section VI, we will see how the $\rho_{\Lambda}$-distribution can be computed analytically from the distribution of the other parameters, so this means that in the $m_{h} \gg \bar{m}$ limit, the only aspect of the potential $V(\phi)$ that significantly affects the cosmological parameter predictions $f_{p}(\mathbf{p})$ is the distribution of curvatures $\eta$ at the peaks.

The situation for Measure B is somewhat more complicated since, as seen in Figure 6, it includes cases where the only substantial period of inflation occurred at a near-inflection point rather than close to maximum, and cases where most of the inflation occurred after our current horizon scale fluctuations had been generated. Figure 8 shows that this favors $n_{s}$-values exceeding unity and gives a broad $\alpha$-distribution in poor agreement with the observed constraint $|\alpha| \lesssim 0.01$ [3]. However, Measure B is seen to share a problematic broad distributions for $\ln Q$. The reason is the same for both measures: Long-lasting inflation requires a tiny $\epsilon$ which can give huge $Q \propto Q_{t} / \sqrt{\epsilon}$, and the value of the $\epsilon$-parameter 55 $e$-foldings before the end of inflation depends sensitively on the second (and for Measure B also higher) derivatives of the potential. 


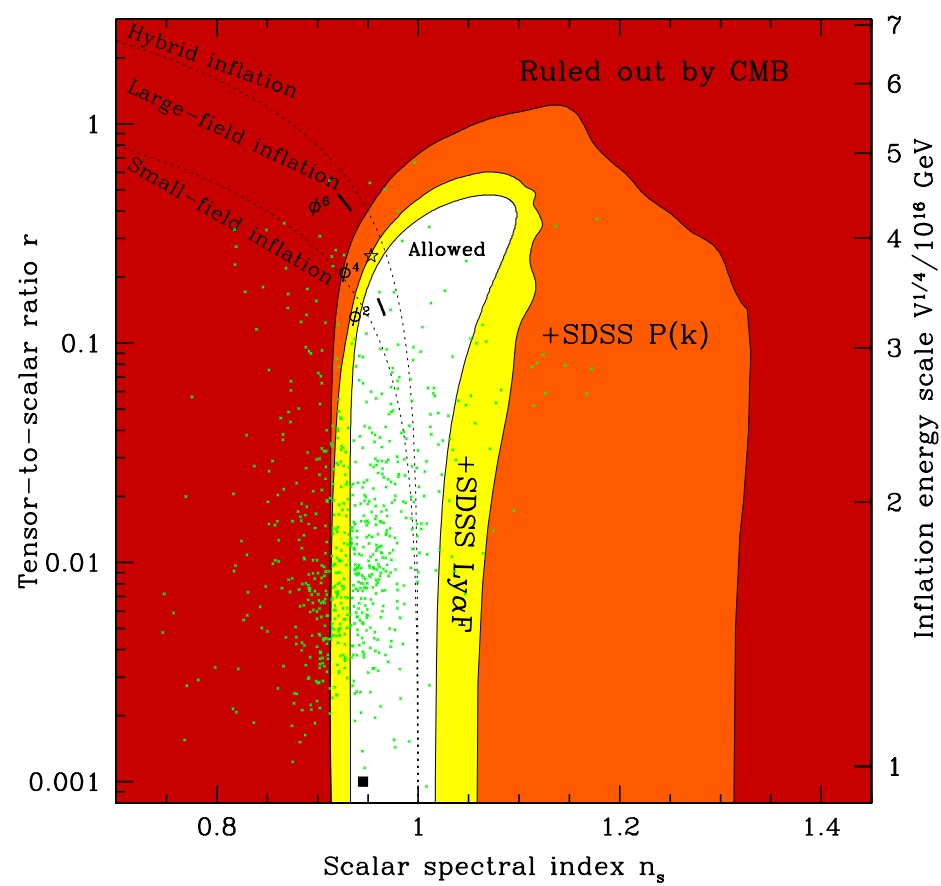

FIG. 13: Constraints and predictions in the $\left(n_{s}, r\right)$-plane. The nested shaded regions are ruled out at $95 \%$ confidence from WMAP alone [1], when adding SDSS galaxy clustering information $[2,86]$ and when also adding SDSS Lyman $\alpha$ Forest information [3]. The green/grey points are the predictions from our simulations with $m_{h}=m_{\mathrm{Pl}}$ and Measure A. The two dotted curves delimit the three classes of inflation models known as small-field, large-field and hybrid models. Some single-field inflation models make highly specific predictions in this plane as indicated. From top to bottom, the figure shows the predictions for $V(\phi) \propto \phi^{6}$ (line segment; ruled out), $V(\phi) \propto \phi^{4}$ (star; ruled out), $V(\phi) \propto \phi^{2}$ (line segment, $m_{h} \gg$ $m_{\mathrm{Pl}}$; still allowed), and $V(\phi) \propto 1-\left(\phi / \phi_{*}\right)^{4}$ (horizontal line segment with $r \sim 10^{-3}$; still allowed), assuming $N=64$ for the $\phi^{4}$ model and $50<N<60$ for the others as per [36].

\section{F. The intermediate case $m_{h} \sim \bar{m}$ : Planck-scale inflation}

Above we saw that models with both $m_{h} \gg \bar{m}$ and $m_{h} \ll \bar{m}$ face severe problems, the former theoretically and the latter observationally. Let us therefore briefly discuss the third option, $m_{h} \sim \bar{m}$, i.e., that the horizontal scale of the inflaton potential is of order the Planck scale.

This is the most complicated of the three cases: the predictions $f_{p}(\mathbf{p})$ will depend on the full details $V(\phi)$, not merely on the peaks or troughs, and none of the abovementioned analytic approximations hold. The only relation between the observables that still holds is therefore the familiar slow-roll consistency relationship $n_{t}=-r / 8$. To make interesting predictions for this case, it is thus crucial to have a physically motivated model for $V(\phi)$, which we currently lack. We will therefore limit this section to one interesting feature which should be rather generic.
Figure 13 compares the predictions the spectral index $n_{s}$ and the tensor-to-scalar ratio $r$ for about $10^{3}$ of our $m_{h}=m_{\mathrm{Pl}}$ simulations with Measure A. The observational constraints will continue to improve dramatically in coming years - for instance, the completed SDSS survey combined with the Planck CMB measurements have been forecast [85] to measure both of these two parameters to an accuracy of about 0.01 . For $n_{s}$, this means that all three of the cases we have considered $\left(m_{h} \ll \bar{m}, m_{h} \sim\right.$ $\bar{m}$ and $m_{h} \gg \bar{m}$ ) generically predict departures from scale-invariance $\left(n_{s}=1\right)$ that should soon be detectable. As regards gravitational waves, the $r$-predictions are seen to scatter up to $r$-values greatly exceeding 0.01 , with the mean being at the readily detectable level $r \approx 0.03$. This bodes well for upcoming CMB polarization missions aiming to detect $B$-polarization.

Both of these qualitative predictions for the $m_{h} \sim m_{\mathrm{Pl}}$ case (good prospects for detecting gravitational waves and lack of scale invariance) are rather generic, since we have seen that they with a greater margin $\left(n_{s}=0.96\right.$ and $r \approx 0.15)$ for essentially any potential when $m_{h} \gg m_{\mathrm{Pl}}$.

\section{G. The effect of quantum diffusion and unbounded potentials}

Our calculations above ignored the effect of quantum diffusion [32] and did not include unbounded potentials where $V \rightarrow \infty$ - let us briefly comment on these two issues.

For these calculations, diffusion makes essentially no difference for Measure B and only a minor difference for Measure A, changing the half-basin weights by factors of order unity. Specifically, quantum diffusion is negligible on parts of the inflaton potential producing fluctuations $Q \ll 1[32]$. For the calculations in this section, diffusion is therefore important only right near the top of peaks in $V(\phi)$ (where we start with Measure A), and will then have the effect of giving roughly equal odds for rolling down from this peak to the left and to the right. In other words, including it numerically would merely readjust the weights of each pair of half-basins separated by a peak by a factor of order unity, making the two weights equal rather than proportional to their length.

Having the potential blow up $(V \rightarrow \infty)$ in places is either irrelevant or observationally ruled out, depending on the horizontal mass scale $m_{h}$. If $m_{h} \gg \bar{m}$, then as we saw above, the observable cosmological parameters depend only the behavior of $V$ near its minima and are unaffected by how the field rolled there (except in so far as it affects the inter-basin weighting). If $m_{h} \ll \bar{m}$, then 55-e-foldings before the end of inflation, $\phi$ was most likely either exponentially close to a maximum of $V$ or extremely high up a $V \rightarrow \infty$ hill. Although our lack of a theory of quantum gravity precludes us from calculating predictions when $V(\phi) \gg \bar{m}^{4}$, even the less extreme case $V(\phi) \sim \bar{m}^{4}$ gives $Q_{t} \sim 1$, i.e., tensor modes producing large-scale unpolarized CMB fluctuations about 5 orders 
or magnitude larger than the current observational limits.

\section{CONDITIONING ON REFERENCE OBJECTS}

Above we computed probability distributions for the cosmological parameter vector $\mathbf{p}$ for various potentials and measures. However, as discussed in Section III, these distributions (Figure 7 and Figure 8) are not the final theoretical predictions that we are entitled to confront with cosmological observation, since they do not include selection effects and hence lack the appropriate weight function $w(\mathbf{p})$ in equation (29). They implicitly used protons or thermalized physical volume as reference objects, but the vantage points from which we make our observations are not a random protons but highly unusual protons (on planets, etc.). Instead, we can think of distributions like those in Figure 7 and Figure 8 as the raw probability distributions that come out of inflation, to be modulated by conditioning on whatever is appropriate to condition on $[46,47]$. To make this more explicit, let us rewrite equation (29) as

$$
f_{p}(\mathbf{p})=f_{p}^{\mathrm{inf}}(\mathbf{p}) w(\mathbf{p}),
$$

defining $f_{p}^{\text {inf }}(\mathbf{p}) \equiv\left\langle\left\langle f_{p}(\mathbf{p} ; \boldsymbol{\Phi}, V)\right\rangle_{\boldsymbol{\Phi}}\right\rangle_{V}$ as the raw parameter distribution emerging from inflation. These two parts of the problem (computing $f_{p}^{\text {inf }}$ and computing $w$ ) thus decouple completely, simply being multiplied at the end. Likewise, the resulting distribution $f_{p}$ for some rudimentary reference objects (protons, say) simply gets multiplied by an additional weight function if we compute the distribution $f_{p}$ for more complex reference objects made of the rudimentary ones (galaxies, say). We will therefore explore only a limited set of reference objects in this section, making various simplifying approximations, leaving a more thorough exploration of weight functions for future work. Specifically, we will explore various combinations of the following weight factors:

$$
\begin{aligned}
w_{\mathrm{vol}}(\mathbf{p}) & \propto e^{3 N_{\mathrm{tot}}} \\
w_{\text {halo }}(\mathbf{p}) & \approx \operatorname{erfc}\left[\frac{0.1 \rho_{\Lambda}^{1 / 3}}{\xi^{4 / 3} Q}\right], \\
w_{N}(\mathbf{p}) & \approx \theta\left[N_{\mathrm{tot}}-N_{\text {gal }}(Q)\right], \\
w_{Q}(\mathbf{p}) & =\text { broad function around } Q \sim 10^{-5},
\end{aligned}
$$

where $\xi \equiv \xi_{\mathrm{b}}+\xi_{\mathrm{c}}+\xi_{\nu} \approx 3.3 \times 10^{-28}$ (see Table 1 ) is the matter-to-photon ratio in Planck units. $w_{\mathrm{vol}}(\mathbf{p})$ is simply the factor by which inflation expands the volume. $w_{\text {halo }}(\mathbf{p})$ is the fraction of all protons that end up gravitationally bound in nonlinear objects (in dark matter halos), so including the $w_{\text {halo-factor in } w(\mathbf{p}) \text { corresponds }}$ to narrowing the class of reference objects to gravitationally bound protons. If the reference objects are galaxies, then the $w_{N}$-factor and the lower cutoff in the $w_{Q}$-factor are arguably required as well. As described below, the
$w_{N}$-factor reflects the fact that long-lived galaxies are only formed if the number of e-foldings exceeds

$$
N_{\text {gal }}(Q) \approx \ln \left(\frac{\rho_{\text {inf }}^{1 / 6} \rho_{\text {reheat }}^{1 / 12}}{\xi Q^{1 / 2}}\right) .
$$

- otherwise, pre-inflationary fluctuations cause dark matter halos to be engulfed by black holes. The lower cutoff in the $w_{Q}(Q)$-factor reflects gas cooling physics related to galaxy formation and the upper cutoff incorporates effects that are relevant if the reference objects are stable planets or observers [87].

Since the inflationary parameters $N_{\text {tot }}, Q$ and $\rho_{\Lambda}$ upon which equations (57)-(60) depend are correlated with our other five parameters, applying these selection effects modifies the observational predictions for all eight inflationary parameters in Table 1 (and also for $\xi$ if this can vary from basin to basin). Arguably our most striking result in this section will be that when taking the selection effects into account, broad classes of inflation models are ruled out by the low observed CMB fluctuation amplitude $Q \sim 2 \times 10^{-5}$ - we will refer to this as the smoothness problem below.

In the next four subsections, we will derive and discuss the weight factors in equations (57)-(60). We will then discuss the implications in sections VIE and VIF. Figure 1 shows an example of the final outcome of these calculations: inflationary predictions with these weight factors (selection effects) included.

\section{A. Volume weighting and conditioning on protons}

Early on, the number density of quarks (later to end up bound into protons and neutrons) is comparable and proportional to the number density of photons $n_{\gamma}$. Then matter and antimatter annihilate, leaving only a small residual proton density $n_{p}=\eta n_{\gamma}$, where the baryon-tophoton ratio $\eta \approx 6 \times 10^{-10}$ (in terms of the parameters in Table $\left.1, \eta=\xi_{\mathrm{b}} / m_{p}\right)$. As long as the physics of baryogenesis that determines $\eta$ has nothing to do with inflation, the number of protons $N_{p}$ in a given comoving region of space that had volume $V_{\text {reh }}$, temperature $T_{\text {reh }}$ and density $\rho_{\text {reh }}$ at the end of reheating is thus given by

$$
N_{p}=V_{\mathrm{reh}} n_{p} \propto V_{\mathrm{reh}} n_{\gamma} \propto V_{\mathrm{reh}} T_{\mathrm{reh}}^{3} \propto V_{\mathrm{reh}} \rho_{\mathrm{reh}}^{4 / 3} .
$$

If the reheat density is independent of the basin into which we roll down (hence constant across our ensemble) and $\rho \propto a^{-3}$ during reheating as usual [36], then the number of protons is thus proportional to the physical volume at the end of inflation. The key question is whether this justifies including the volume factor of equation (57) in the weight function $w(\mathbf{p})$ of equation (29).

When comparing finite volumes, the answer appears to be a clear yes, since there will be only a finite number of protons and hence no ambiguities associate with their ordering in equation (26). This implies that when comparing finite and infinite pockets, the finite ones get zero 
statistical weight since the infinite ones contain almost all reference objects. In terms of the previous section, it argues for Measure A over Measure B. When comparing two different infinite volumes, the answer is less clear, and is equivalent to specifying the inter-pocket weighting. Are all infinite volumes equal, or are some more infinite than others? We will return to this important question in Section VII.

\section{B. $w_{\text {halo }}$ : conditioning on halos}

This particular selection effect (that we are observing from a region of space where gravitationally bound object have been able to form) was the one used to obtain the classic anthropic upper bounds on the dark energy density $\rho_{\Lambda}[61,64,67-71]$. Its inclusion is clearly not optional when testing theories where $Q, \rho_{\Lambda}$ or $\xi$ vary, and it is more elegant than many other selection effects in that it is physically clean, involving only well-understood gravitational physics and no uncertainties related to the formation of galaxies, planets of observers. Any future definition of an observer is likely to require the inclusion of the $w_{\text {halo-factor, since no complex objects whatsoever }}$ (let alone observers) ever form in the linear regime.

The first mass scales $M$ (if any) to go nonlinear are the smallest, which enter the horizon well before matterradiation equality with a fluctuation amplitude $\sigma_{*}(M) \sim$ $Q$ and then grow over time due to gravitational instability as (see Appendix A of [88])

$$
\sigma(M) \approx\left[1+\frac{3}{2}\left(\frac{\rho_{\mathrm{m}}^{\mathrm{eq}}}{\rho_{\Lambda}}\right)^{1 / 3} G_{\Lambda}\left(\frac{\rho_{\Lambda}}{\rho_{\mathrm{m}}}\right)\right] \sigma_{*}(M),
$$

where the dimensionless function

$$
G_{\Lambda}(x) \approx x^{1 / 3}\left[1+\left(\frac{x}{G_{\infty}^{3}}\right)^{0.795}\right]^{-\frac{1}{3 \times 0.795}}
$$

describes how fluctuations grow as the cosmic scale factor $a$ as long as dark energy is negligible $\left(G_{\Lambda}(x) \approx x^{1 / 3}=\right.$ $\left(\rho_{\Lambda} / \rho_{\mathrm{m}}\right)^{1 / 3} \propto \rho_{\mathrm{m}}^{-1 / 3} \propto a$ for $\left.x \ll 1\right)$ and then asymptote to a constant value as $a \rightarrow \infty$ and dark energy dominates: $G_{\Lambda}(x) \rightarrow G_{\infty}$ as $x \rightarrow \infty$, where

$$
G_{\infty} \equiv \frac{5 \Gamma\left(\frac{2}{3}\right) \Gamma\left(\frac{5}{6}\right)}{3 \sqrt{\pi}} \approx 1.43728 .
$$

Equation (64) assumes negligibly small neutrino masses, $\rho_{\Lambda} \geq 0$ and a flat Universe - we will discuss the $\rho_{\Lambda}<0$ case below and include the effect of spatial curvature in Section VIC 2. Neutrino masses lower the $\rho_{\Lambda}$-predictions marginally $[88,89]$, but within the stringent current observational limits [3], they have negligible effect on the qualitative results of this paper.

In Planck units, the matter density at matter-radiation equality is

$$
\rho_{\mathrm{m}}^{\mathrm{eq}}=n_{\gamma}^{\mathrm{eq}} \xi=\frac{2 \zeta(3)}{\pi^{2}} T_{\mathrm{eq}}{ }^{3} \xi
$$

where the Riemann zeta function $\zeta(3) \approx 1.20206$ and the matter-radiation equality temperature is

$$
T_{\mathrm{eq}}=\frac{30 \zeta(3)}{\pi^{4}}\left[1+\frac{21}{8}\left(\frac{4}{11}\right)^{4 / 3}\right]^{-1} \xi \approx 0.220189 \xi,
$$

where the second term in square brackets reflects the contributions from neutrinos to the radiation density. Equation (63) thus shows that the net fluctuation growth is controlled by the factor

$$
\left(\frac{\rho_{\mathrm{m}}^{\mathrm{eq}}}{\rho_{\Lambda}}\right)^{1 / 3} \approx 0.13751 \frac{\xi^{4 / 3}}{\rho_{\Lambda}^{1 / 3}},
$$

observed to be $3215 \pm 639[2,88]$, which is the factor by which the universe expanded between matter domination (when growth effectively begins) and dark energy domination (when growth effectively ends).

We approximate the fraction of matter collapsed into dark matter halos by the standard Press-Schechter formalism [90], which gives

$$
w_{\text {halo }}(\mathbf{p})=\operatorname{erfc}\left[\frac{\delta_{c}}{\sqrt{2} \sigma(M)}\right] \approx \operatorname{erfc}\left[\frac{2.7046 \rho_{\Lambda}^{1 / 3}}{\xi^{4 / 3} \sigma_{*}(M)}\right],
$$

where $\delta_{c}=(9 / 5) 2^{-2 / 3} \approx 1.63$ (not 1.69$)$ is the fluctuation threshold corresponding to the infinite future (see [68] and appendix D.4 of [88]). In the second step of equation (69), we used equations (64), (65) and (68) and took the limit $t \rightarrow \infty$, i.e., $\rho_{\mathrm{m}} \rightarrow 0$. To derive equation (58) from equation (69), all that remains is to relate the initial fluctuation amplitude $\sigma(M)$ to $Q$. For the small scales relevant to galaxy halos, the ratio $\sigma(M) / Q$ is a constant of order unity which depends very weakly (roughly logarithmically) on the mass scale $M$. To be specific, we use the observed ratio $\sigma(M) / Q \approx 29$ corresponding to a galactic halo scale $M=10^{12} M_{\odot}[2,88]$. This particular choice of scale is irrelevant to the qualitative conclusions we will draw in this paper, since slight variations in the numerical prefactor 0.1 in equation (58) are dwarfed by the order-of-magnitude changes in $\rho_{\Lambda}$ and $Q$.

Equation (58) applies to the case $\rho_{\Lambda} \geq 0$ and gives $w_{\text {halo }} \approx 0$ if $\rho_{\Lambda} \gg \xi^{4} Q^{3}$. If $\rho_{\Lambda} \ll-\xi^{4} Q^{3}$, then space will recollapse before any reference objects have had time to form $[61,64,67-71]$. Although the physics is more complicated than for the $\rho_{\Lambda}>0$ case (since fluctuations $d o$ have time to go nonlinear before the Big Crunch), there is no indication that the constraints on negative values of $\rho_{\Lambda}$ are much weaker than those on positive values. This implies that

$$
w_{\text {halo }}(\mathbf{p}) \approx\left\{\begin{array}{l}
1 \text { if }\left|\rho_{\Lambda}\right| \ll \xi^{4} Q^{3}, \\
0 \text { if }\left|\rho_{\Lambda}\right| \gg \xi^{4} Q^{3},
\end{array}\right.
$$

in Planck units, which is all we need for the qualitative conclusions of the present paper. The origin of this result is that the parameter combination $\rho_{\Lambda} / \xi^{4} Q^{3}$ is simply the 
ratio of the dark energy density to the matter density at the epoch when halos would form if $\rho_{\Lambda}=0$. Regardless of the sign of $\rho_{\Lambda}$, dark energy domination is when time starts running out for the formation of reference objects: either quite literally for the $\rho_{\Lambda}<0$ case (because space recollapses) or for the $\rho_{\Lambda}>0$ case (because fluctuation growth stops).

\section{C. $w_{N}$ and $w_{Q}$ : conditioning on galaxies}

Above we computed the weight factor $w_{\text {halo }}$ that entered when the reference objects were dark matter halos. Let us now compute the additional weight factors that enter when the reference objects are galaxies. We will see that they depend primarily on $N_{\text {tot }}$ and $Q$.

\section{When galaxy-scale fluctuations were generated}

Consider a comoving Hubble volume (either ours or a smaller one) that left the horizon at $a_{\text {exit }}$ during inflation (open triangle in Figure 3, say) and reentered at $a_{0}$ during matter domination (filled triangle, say). As discussed in Section II and Appendix A, these two points are connected by $a_{0}^{2} \rho\left(a_{0}\right)=a_{\text {exit }}^{2} \rho\left(a_{\text {exit }}\right)$. By making the standard assumptions that $\rho \stackrel{\propto}{\sim} a^{-3}$ during matter domination, $\rho \stackrel{\propto}{\sim} a^{-4}$ during radiation domination, $\rho \stackrel{\sim}{\sim} a^{-3}$ during reheating and $\rho \underset{\sim}{\sim} a^{0}$ during inflation (the five above relations are illustrated in Figure 3 by lines of slope $-3,-4,-3,0$ and -2 ), one readily derives the following expression for the number of e-foldings $N_{0}$ between horizon exit and the end of inflation [36]:

$$
N_{0}=\ln \frac{a_{\mathrm{end}}}{a_{\mathrm{exit}}} \approx \ln \left[\frac{\rho_{\mathrm{infl}}^{1 / 6} \rho_{\text {reheat }}^{1 / 12}}{\rho_{\mathrm{eq}}^{1 / 4}}\left(\frac{a_{0}}{a_{\mathrm{eq}}}\right)^{1 / 2}\right] .
$$

Working in Planck units, combining equation (66) with equation $(67)$ shows that $\rho_{\text {eq }} \sim \xi^{4}$. We use the symbol $\sim$ to indicate that we are ignoring numerical factors of order unity for simplicity, since equation (71) shows that they affect $N_{0}$ only logarithmically. The number of e-foldings $N_{\text {eq }}$ between horizon exit of the matter-radiation equality scale (open square in Figure 3) and the end of inflation (five-pointed star) is thus simply

$$
N_{\text {eq }} \approx \ln \left(\frac{\rho_{\text {infl }}^{1 / 6} \rho_{\text {reheat }}^{1 / 12}}{\xi}\right) .
$$

Fluctuations that entered the horizon before matterradiation equality grew during matter domination with amplitude of order $\left(a / a_{\mathrm{eq}}\right) Q$, so the first nonlinear objects formed at the epoch $a \sim a_{\text {eq }} / Q$. Since galaxies cannot form before this time, the number of e-foldings $N_{\text {gal }}$ between horizon exit of the galaxy scale and the end of inflation is therefore at least

$$
N_{\text {gal }} \approx \ln \left(\frac{\rho_{\mathrm{inf}}^{1 / 4} R^{1 / 12}}{\xi Q^{1 / 2}}\right)
$$

where $R \equiv \rho_{\text {reheat }} / \rho_{\text {infl }}$ equals unity if reheating is instantaneous and decreases if the duration of the reheating epoch grows. For canonical values $\xi \approx 3.3 \times 10^{-28}$, $Q \approx 2 \times 10^{-5}$ and $\rho_{\text {infl }} \sim\left(10^{16} \mathrm{GeV}\right)^{4} \sim 10^{-12}$, we thus obtain $N_{\text {gal }} \approx 55$ for instant reheating $(R=1)$. Lowering the reheat energy to the extreme value $1 \mathrm{TeV}$ (arguably about the lowest energy that is still observationally allowed) gives $N_{\text {gal }} \approx 65$ and, conversely, lowering the inflation energy scale reduces $N_{\text {gal }}$.

\section{The black hole constraint on galaxy formation}

In the context of galaxy formation, there are two sources of fluctuations, one good and one bad:

1. Inflation-generated fluctuations that are of order $Q$ when they enter the horizon.

2. Pre-inflationary fluctuations that we assume to be of order unity when they enter the horizon.

Consider what happens in a spatial region that inflated by only a finite number of e-foldings $N_{\text {tot }}$ that exceeds $N_{\text {eq }} \sim 50$, say. Since inflation diluted away sub-horizon pre-inflationary fluctuations, the first fluctuations to enter the horizon after matter-radiation equality have amplitude $Q$, then grow $\propto a$ due to gravitational instability as long as $\rho_{\Lambda}$ is negligible, perhaps forming galaxies if they are able to grow enough to go nonlinear. If $\rho_{\Lambda}=0$, then the horizon will keep growing until, eventually, preinflationary fluctuations of order unity start entering the horizon. This is bad news, since once the current horizon scale has gone nonlinear, the horizon volume lies within its own Schwarzschild radius and will recollapse into a giant black hole on the local Hubble timescale, i.e., roughly by the time the cosmic age has doubled. ${ }^{10}$ Even if the first order-unity fluctuations to enter the horizon happen to be negative and void-like, still larger-scale fluctuations will keep entering and ensure that our local spatial patch is engulfed by a black hole before long. In summary, horizon-sized black holes form at the epoch

$$
a_{\mathrm{bh}} \sim a_{\mathrm{eq}} e^{2\left(N_{\mathrm{tot}}-N_{\mathrm{eq}}\right)}
$$

when pre-inflationary fluctuations start entering the horizon.

If our reference objects are merely nonlinear structures, then fluctuations of the above-mentioned types 1 and 2 are equally useful for producing them, and there is no constraint whatsoever on $N_{\text {tot }}$, the duration of inflation. Indeed, no inflation at all is needed, since objects

10 Note that fluctuations of order unity in a flat FriedmanRobertson-Walker background only form black holes if they are on or above the current horizon scale. In contrast, fluctuations with $Q \ll 1$ grow to order unity only much later, when the current horizon scale is vastly larger than the fluctuation scale. 
would be nonlinear from the outset. In contrast, reference objects such as galaxies, planets and observers all require extra time to form. Specifically, they require a period of peace and quiet after their parent dark matter halo has gone nonlinear, and will not have time to form if their parent halo is promptly swallowed by a black hole. Such reference objects will therefore only form if halos form substantially before the epoch $a_{\mathrm{bh}}$, i.e., if $N_{\text {tot }} \gtrsim N_{\text {gal }}(Q)$. This constraint on the duration of inflation is embodied by equation (59).

None of the qualitative conclusions in this paper depend on whether $N_{\text {gal }}=50,60$, or some other number of order $10^{2}$. Since the dependence of $N_{\text {gal }}$ on $Q$ is only logarithmic, i.e., much weaker than that of the $w_{\text {halo factor }}$ of equation (58), we therefore made the crude approximation $N_{\text {gal }}=55$ for simplicity in Section $\mathrm{V}$.

\section{3. $w_{Q}$ : The cooling constraint on galaxy formation}

To end up in a galaxy, a proton must first fall into a dark matter halo (a fraction $w_{\text {halo }}$ factor does this), then avoid promptly being engulfed by a black whole (a fraction $w_{N}$ avoids this). Next, the gas to which it belongs needs to cool efficiently enough to be able to contract, become self-gravitating and form stars (see, e.g., [91-94]). This problem is analyzed in terms of fundamental physical constants in [87]. If we decrease $Q$, then dark matter halos form later and have lower escape velocities, hence heating infalling gas to lower temperatures where cooling is less efficient, causing the weight function $w_{Q}(Q)$ to fall off towards very low $Q$-values. After changing variables to reflect the notation in this paper, equation (11) in [87] states that $w_{Q}(Q) \approx 0$ unless

$$
Q \gtrsim 0.2 \alpha^{-1} \ln \left[\alpha^{-2}\right]^{-16 / 9} m_{e}^{4 / 3} \eta^{-2 / 3} \xi^{-2 / 3} \sim 10^{-6},
$$

where $\alpha \approx 1 / 137.036$ is the fine structure constant and $m_{e} \approx 4.2 \times 10^{-23}$ is the electron mass in Planck units.

\section{D. $w_{Q}$ : conditioning on stable planets}

If the reference objects are observers who, like us, have required billions of years of evolution on a planet, we need to include an additional weight factor $w_{Q}(Q)$ incorporating the fraction of the protons in galaxies that end up in planets in long-lived stable orbits. As discussed in [87], if we increase $Q$, then galaxies form earlier with higher stellar density, increasing the rate of catastrophic orbit disruptions from near encounters with other stars. Equation $(19)$ in [87] states that $w_{Q}(Q) \approx 0$ unless

$$
Q \lesssim 10^{-4}
$$

In summary, combining equations (75) and (76) suggests that $w_{Q}(Q) \sim 0$ unless $10^{-4} \lesssim Q \lesssim 10^{-6}$. However, as detailed in [87], the physical assumptions underlying the calculations of $w_{Q}$ are not nearly as clean and clear-cut as those underlying, say, the $w_{\text {halo-factor, so this conclusion }}$ should be taken with a healthy grain of salt, and deserves further investigation.

\section{E. Predictions for the horizon problem}

In the four preceding subsections, we derived the weight factors in equations (57)-(60). Let us now explore their implications, starting in this section with equation (59).

Inflation solves the horizon problem if $N_{\text {tot }} \gtrsim N_{0}$, so that all fluctuations that have entered our horizon so far have inflationary rather than pre-inflationary origin. In Section A 2, we saw that if inflation lasts for an additional few e-foldings, it solves the flatness problem as well: since $N_{\text {before }}=N_{\text {tot }}-N_{0}$, we found that $N_{\text {before }} \gtrsim 0$ solves the horizon problem and $N_{\text {before }} \gtrsim 3$ reproduces the observed spatial flatness.

One of the original motivations for the early work on inflation was that it could solve the horizon problem and the flatness problem [80-84] (for certain potentials). To what extent can we make the prediction that inflation does solve these problems?

When the total number of e-foldings $N_{\text {tot }}$ varies across the ensemble, it will generally exceed $N_{0}$ in some spatial regions but not in others. For observations made from galaxies, we automatically have $N_{0} \geq N_{\text {gal }}$, but the existence of galaxies guarantees only that $N_{\text {tot }}>N_{\text {gal }}$, not that $N_{\text {tot }}$ also exceeds the larger quantity $N_{0}$ corresponding to the current observation time. The probability of observing the horizon problem to be solved is thus the probability of observing $N>N_{0}$ given that $N>N_{\text {gal }}$, i.e.,

$$
P_{\text {horizon }}=P\left(N_{\text {tot }}>N_{0} \mid N_{\text {tot }}>N_{\text {gal }}\right)=\frac{P\left(N_{\text {tot }}>N_{0}\right)}{P\left(N_{\text {tot }}>N_{\text {gal }}\right)} \text {. }
$$

In Section $\mathrm{V}$, we found that Measure A predicted $N_{\text {tot }}=$ $\infty$, automatically solving the horizon and flatness problems, whereas our other measure gave a probability distribution for $N$ falling off roughly as $N_{\text {tot }}^{-\gamma}$ with $\gamma \approx 3$ in the worst-case scenario $m_{h} \ll \bar{m}$ (plotted in Figure 12). Equation (71) shows that $N_{0}-N_{\text {gal }}=\frac{1}{2} \ln \left(1+z_{\text {gal }}\right) \sim 1$, where $z_{\text {gal }}$ is the galaxy formation redshift. For $N_{\text {gal }}=$ 55 , equation (77) therefore gives

$P_{\text {horizon }}=\left\{\begin{array}{l}1 \text { with volume-weighting, } \\ \left(\frac{N_{0}}{N_{\text {gal }}}\right)^{-\gamma} \approx 1-\frac{\gamma}{2} \frac{\ln \left(1+z_{\text {gal }}\right)}{N_{\text {gal }}} \sim 95 \% \text { otherwise. }\end{array}\right.$

For either measure, inflation thus predicts that the horizon problem is solved from the vantage point of most galaxies. If we were to retrodict whether our own horizon-scale fluctuations were inflationary rather than pre-inflationary, varying $z_{\text {gal }}$ over the observationally rather extreme range $1<z_{\text {gal }}<100$ would give $87 \%<P_{\text {horizon }}<98 \%$, so our qualitative conclusion that 
$P_{\text {horizon }} \sim 1$ is independent of uncertainties regarding what to take as the present epoch. We can also make this argument in a less anthropocentric way, noting that since the $w_{\text {halo-factor tends to postpone dark energy domina- }}$ tion tends to only slightly after galaxy formation, the horizon stops growing and precludes observers from observing more than a few e-foldings beyond $N_{\text {gal }}$ no matter how long they wait.

In conclusion, none of the inflation models we have considered are ruled out by the horizon problem. In Section $\mathrm{V}$, we saw that they were not ruled out by the flatness problem either.

\section{F. The smoothness problem and other implications}

If a physics problem involves two vastly different scales, this fact can often be used to simplify it. We will now see that precisely this happens in our inflationary prediction problem, because the inflation density vastly exceeds the dark energy density $\xi^{4} Q^{3} m_{\mathrm{Pl}}^{4}$ allowed by equation (70). Conveniently, this will allow us to deal analytically with the $\rho_{\Lambda}$-parameter and dispense with it once and for all.

In Section V, we saw that inflation typically ended at a density $\rho_{\text {end }} \sim m_{v}{ }^{4}$ for $m_{h} \lesssim \bar{m}$ and $\rho_{\text {end }} \sim\left(m_{h} / \bar{m}\right)^{2} m_{v}{ }^{4}$ for $m_{h} \gtrsim \bar{m}$. For any relevant values of $Q, m_{h}$ and $m_{v}$, both of these two densities vastly exceed the density scale $\xi^{4} Q^{3} m_{\mathrm{Pl}}^{4} \sim 10^{-110} Q^{3} m_{\mathrm{Pl}}^{4}$ above which equation (58) cuts off the statistical weight (no halos form). The lower limit on negative $\rho_{\Lambda}$-values from requiring reference objects to form before space recollapses is of comparable magnitude. This means that the only part of the probability distribution for $\rho_{\Lambda}=V\left(\phi_{\text {stop }}\right)=m_{v}{ }^{4} f\left(\phi_{\text {stop }} / m_{h}\right)$ that matters is that in a region around zero which is way smaller in magnitude than any relevant physical scales. Since the dimensionless inflaton potential $f$ by definition varies of order unity and zero is not in any way a special value for a random minimum of a complicated function, it follows that we can for all practical purposes take $\rho_{\Lambda}$ to have a uniform distribution, uncorrelated with all the other parameters. This prediction is confirmed by our numerical results and illustrated by the $\rho_{\Lambda}$-histograms in figures $(7)$ and (8).

\section{Marginalizing over $\rho_{\Lambda}$}

Since $w_{\text {halo }}$ is the only one of the weight functions from equations (57)-(60) that depends on $\rho_{\Lambda}$, this result means that we can once and for all marginalize over $\rho_{\Lambda}$ analytically and eliminate it from the discussion of our other cosmological parameters. The resulting parameter dis- tribution observed from a random halo is

$$
\begin{aligned}
f_{p}^{\text {halo }}(\mathbf{p}) & \propto \int_{-\infty}^{\infty} f_{p}^{\text {inf }}(\mathbf{p}) w_{\text {halo }}(\mathbf{p}) d \rho_{\Lambda} \\
& \approx \int_{-\infty}^{\infty} f_{p}^{\inf }(\mathbf{p})_{\mid \rho_{\Lambda}=0} g\left(\frac{\rho_{\Lambda}}{\xi^{4} Q^{3}}\right) d \rho_{\Lambda} \\
& \propto \xi^{4} Q^{3} f_{p}^{\text {inf }}(\mathbf{p})_{\mid \rho_{\Lambda}=0} .
\end{aligned}
$$

On the second line, we used the above uniform distribution result to approximate the first factor $f_{p}^{\text {inf }}(\mathbf{p})$ by its value at $\rho_{\Lambda}=0$ because it is $\rho_{\Lambda}$-independent whenever the second factor is appreciably nonzero. ${ }^{11}$ Here we also reexpressed $w_{\text {halo }}(\mathbf{p})$ as $g\left(\rho_{\Lambda} / \xi^{4} Q^{3}\right)$ to emphasize that the final result in equation (79) applies to much more general weight functions $w_{\text {halo }}(\mathbf{p})$ than of that of equation (58) - which corresponds to the particular case $g(x)=\operatorname{erfc}\left(0.1 x^{1 / 3}\right)$. Replacing the Press-Schechter formula by the more accurate approximation of Sheth \& Tormen [95] would merely modify the functional form of $g(x)$, not the final result in equation (79). More importantly, modifying $w_{\text {halo }}(\mathbf{p})$ to explicitly give zero weight to models where space recollapses before reference objects form (which is necessary to prevent the integral in equation (79) from diverging as $\rho_{\Lambda} \rightarrow-\infty$ ) would again simply modify the functional form of $g(x)$, this time to ensure that the condition $g(x) \approx 0$ for $|x| \gg 1$ held also for negative $x$, as per equation (70).

The $Q^{3}$-factor on the last line of equation (79) poses great difficulties for many inflation models, since it can easily overpower a slight inflationary preference for low $Q$-values in $f^{\inf }(\mathbf{p})$ (Figure 14) and give predictions far exceeding the observed value $Q \sim 2 \times 10^{-5}$. As a concrete illustration of this smoothness problem, consider the models with $m_{h} \lesssim \bar{m}$ from Section V. These were the only models that were natural, in the sense that quantum gravity corrections may preclude larger $m_{h}$-values. We found that all these models predicted a $Q$-distribution with a high tail falling off roughly as $f_{Q}(Q) \propto Q^{-1}$, corresponding to a near-uniform distribution for $\ln Q$, as confirmed numerically in Figure 14. The basic reason for this was that $Q \sim V^{3 / 2} /\left|V^{\prime}\right|$, and that $V^{\prime}$ was often exponentially small. Equation (79) now shows that these models predict a rising tail, with a random halo seeing a distribution $f_{Q}(\mathbf{p}) \propto Q^{2}$ for large $Q$. Quantum diffusion

11 Because our simulations predict the $\rho_{\Lambda}$-distribution to be uniform over a much broader range $\left(\left|\rho_{\Lambda}\right| \ll \rho_{\text {end }}\right)$ than the range forming halos, it would be numerically inconvenient to apply equation (58) to our simulations: it would give a success rate $\ll 10^{-100}$ and require many Hubble times worth of computer simulating to obtain even a single successful model. Requiring $\rho_{\Lambda}=0$ as in equation (79) is of course even less convenient numerically. In practice, we therefore use the result of equation (79) with the constraint $\rho_{\Lambda}=0$ replaced by $0 \leq \rho_{\Lambda}<\rho_{\Lambda}^{*}$, where $\rho_{\Lambda}^{*} \ll \rho_{\text {end }}$. We choose the cutoff $\rho_{\Lambda}^{*}$ sufficiently small that reducing it further has no effect on our predicted distributions, but large enough that we retain a statistically large sample of models. 


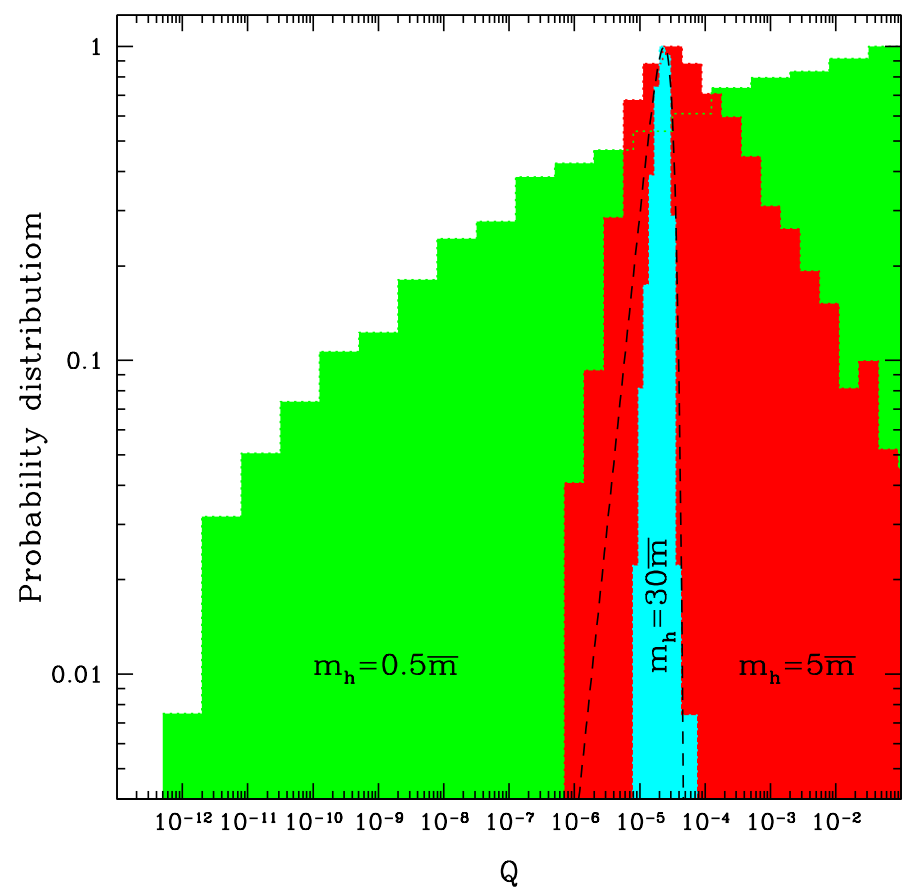

FIG. 14: The smoothness problem is seen to afflict quantumgravitationally "natural" inflation models with $m_{h} \ll m_{\mathrm{Pl}} \approx 5 \bar{m}$, predicting $Q$ way above the observed value $Q \sim 2 \times 10^{-5}$ since $\rho_{\Lambda}$-marginalization multiplies the above distributions by an extra $Q^{3}$-factor. The three histograms are for Measure A and show the distribution of $\lg Q$ for $\rho_{\Lambda} \approx 0$, marginalized over all other cosmological parameters. The $m_{h}=0.5 \bar{m}$ distribution is seen to approach the analytic $m_{h} \rightarrow 0$ prediction of a uniform distribution in the high $\lg Q$ tail, and the $m_{h}=30 \bar{m}$ curve is seen to be approximated by the analytic $m_{h} \rightarrow \infty$ prediction $\propto Q^{2} e^{-\left(Q / Q_{*}\right)^{4}}$ for $Q_{*} \approx 5 m_{v}^{2} / m_{h}$ near the peak (dashed line).

cuts off this distribution at $Q \sim 1$, so $Q$ observed from a random halo would be of order unity. Equation (55) shows that this result is independent of the choice of the vertical energy scale $m_{v}$ unless it is down in the $\mathrm{TeV}$ range - for that extreme case, equations (49) and (51)) show that $\eta_{0} \approx-1$ and $n_{s} \approx-1$.

All these $m_{h} \ll \bar{m}$ models are thus firmly ruled out by the observed measurement $Q \sim 2 \times 10^{-5}$ unless they are rescued by the weight function $w_{Q}(Q) .{ }^{12}$ However, as discussed in [87] and Section VI C 3, the anthropic upper limits on $Q$ from planetary orbit disruptions and other effects appear rather weak, both in terms of physical uncertainties and in terms of the actual numbers. In particular, there is currently no compelling argument for why there would be much fewer observers if $Q$ were say 3 times larger than observed, yet these inflation models predict

12 The $w_{N}$-factor of equation (59) provides essentially no help although it does depend on $Q$ for one of the two measures we studied, the dependence is very weak because of the logarithm and the broad intrinsic width of the $N$-distribution.

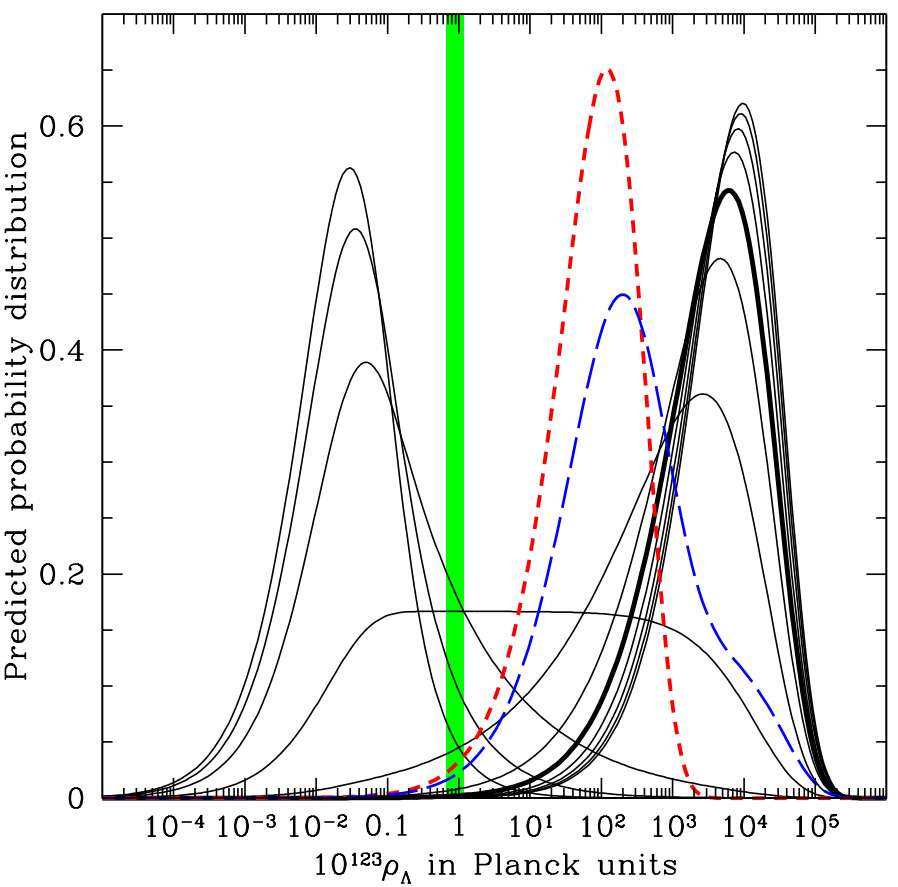

FIG. 15: Predictions for the dark energy density $\rho_{\Lambda}$ for the toy model where reference objects get produced if $10^{-6}<Q<10^{-4}$ and inflation predicts a power law $f_{Q}(Q) \propto Q^{n}$ across this range. Peaking from left to right, the solid curves correspond to $n=-8$, $-7,-6,-5,-4,-3,-2,-1$ (heavy), $0,1,2$ and 3 , respectively. The short-dashed curve shows the case where inflation predicts $Q=2 \times 10^{-5}$ without any scatter, and the long-dashed curve is for the actual $Q$-distribution for $m_{h}=10 \bar{m}, m_{v}=0.01 \bar{m}$ from Figure 1. The shaded band shows the WMAP+SDSS measurement from Table 1.

the $(\ln Q)$-distribution $\propto Q^{3}$ so that this larger $\ln Q$-value would be a priori about 30 times more likely. Having $\ln Q$ up by an order of magnitude (bumping up against the disruption limit of [87]) would be a priori about 1000 times more likely. It would be much more satisfying if this smoothness problem could be solved by a well-motivated inflation model firmly predicting smaller $Q$-values.

Analogously, one can argue that equation (79) rules out any particle physics model for the matter density parameter parameter $\xi$ if it predicts an ensemble with a high- $\xi$ tail shallower than about $\xi^{-5}$, unless galaxy formation can be shown to be strongly inhibited by small increases in $\xi-$ for a discussion of such dark matter issues, see $[46,96]$.

\section{Predictions for $\rho_{\Lambda}$}

Above we marginalized over $\rho_{\Lambda}$ to obtain predictions for the other parameters. Let us now do the opposite, obtaining inflationary predictions for $\rho_{\Lambda}$. The inflationary prediction for the dark energy probability distribution $f_{\Lambda}\left(\rho_{\Lambda}\right)$ is obtained by marginalizing $f_{p}(\mathbf{p})$ from equa- 
tion (56) over the other seven parameters in the $\mathbf{p}$-vector, i.e.,

$$
f_{\Lambda}\left(\rho_{\Lambda}^{\prime}\right) \propto \int f^{\inf }(\mathbf{p}) w(\mathbf{p}) \delta\left(\rho_{\Lambda}-\rho_{\Lambda}^{\prime}\right) d^{8} p .
$$

Above we found that $f^{\inf }(\mathbf{p})$ was $\rho_{\Lambda}$-independent over the narrow range of $\mathbf{p}$-values where $w(\mathbf{p})$ was nonzero. The only $\rho_{\Lambda}$-dependence in equation (80) therefore comes from the weight function $w(\mathbf{p})$, where $\rho_{\Lambda}$ enters in the halo term $w_{\text {halo }}(\mathbf{p})$ of equation (58) and gets coupled with $Q$. We can thus integrate trivially over all parameters except $Q$ in equation (80), obtaining

$$
f_{\Lambda}\left(\rho_{\Lambda}\right) \propto \int_{0}^{\infty} f^{\inf }(Q) w_{Q}(Q) \operatorname{erfc}\left[\frac{0.1 \rho_{\Lambda}^{1 / 3}}{\xi^{4 / 3} Q}\right] d Q
$$

where $f^{\inf }(Q)$ is the raw inflation prediction for $Q$ for $\rho_{\Lambda}=0$ (i.e., $\left.f^{\inf }(\mathbf{p})\right|_{\rho_{\Lambda}=0}$ marginalized over all parameters except $Q$ ), as plotted in Figure 14 and in the $Q$ panels of figures (8) and (7). Since the values $Q_{i}$ in our simulations with $\rho_{\Lambda} \approx 0$ are drawn from the distribution $f^{\text {inf }}(Q)$, we can evaluate this expression in practice as

$$
f_{\Lambda}\left(\rho_{\Lambda}\right) \propto \sum_{i} w_{Q}\left(Q_{i}\right) \operatorname{erfc}\left[\frac{0.1 \rho_{\Lambda}^{1 / 3}}{\xi^{4 / 3} Q_{i}}\right],
$$

where the sum is over those simulations for which $\rho_{\Lambda} \approx 0$. If inflation were to predict that $Q$ took a specific value $Q_{*}$-value (i.e., if $f^{\inf }(Q)=\delta\left(Q-Q_{*}\right)$, as for a onedimensional inflaton potential with only a single minimum that either was symmetric or could only be slowrolled to from one side, then equation (81) would give the classic prediction $\rho_{\Lambda} \lesssim 10^{3} \xi^{4} Q_{*}^{3}[61,64,67-71,87]$ (more specifically, the dashed curve in Figure 15). However, as stressed in [97], degeneracies with other parameters can invalidate such arguments. Sure enough, in the cases we have considered in this paper, the distribution $f^{\text {inf }}(Q)$ has a nonzero width, and equation (81) shows that a tail towards large $Q$-values will give more statistical weight to higher $\rho_{\Lambda}$-values. This important coupling between $\rho_{\Lambda}$ and $Q$ was ignored in the early work predicting $\rho_{\Lambda^{-}}$ distributions. It was emphasized in [87] and recently explored numerically in [98] for some instructive toy models, with the conclusion that it can invalidate anthropic explanations for $\rho_{\Lambda}$ for a broad class of primordial $Q$ distributions. [99] explores how $w \neq-1$ affects this link. Using our results from Section $V$, we can for the first time explore this issue with $Q$-distributions from actual inflation calculations.

Before doing this, however, let us first build some intuition for equation (81). Consider the toy model where $f^{\text {inf }}(\mathbf{p}) \propto Q^{n}$ for some power law index $n$ and the weight function $w_{Q}(Q)=1$ if $Q_{\min } \leq Q \leq Q_{\max }$, vanishing otherwise. Limiting our attention to the case $\rho_{\Lambda} \geq 0$ and performing the integral in equation (81) numerically now gives the curves shown in Figure 15, which are easy to understand intuitively if we define the quantities
$\rho_{\Lambda}^{\min } \equiv \xi^{4} Q_{\min }^{3}$ and $\rho_{\Lambda}^{\max } \equiv \xi^{4} Q_{\max }^{3}$. Since erfc $x \approx 1$ for $x \ll 1$ and erfc $x \approx 0$ for $x \gg 1$, equation (81) shows that $f_{\Lambda}\left(\rho_{\Lambda}\right)$ is constant if $\rho_{\Lambda} \ll \rho_{\Lambda}^{\min }$, vanishes if $\rho_{\Lambda} \gg \rho_{\Lambda}^{\max }$ and is otherwise approximated by

$$
f_{\Lambda}\left(\rho_{\Lambda}\right) \propto \int_{\frac{0.1 \rho_{\Lambda}^{1 / 3}}{\xi^{4 / 3}}}^{Q_{\max }} Q^{n} d Q \stackrel{\propto}{\sim}\left|1-\left(\frac{\rho_{\Lambda}}{\rho_{\Lambda}^{\max }}\right)^{\frac{n+1}{3}}\right| .
$$

Figure 15 shows the distribution of $\lg \rho_{\Lambda}$ rather than $\rho_{\Lambda}$, which introduces an extra $\rho_{\Lambda}$-factor since $d \rho_{\Lambda}=$ $\rho_{\Lambda} d \ln \rho_{\Lambda}$, making the exponent of the second term of equation (83) equal to $(n+4) / 3$. Whether $n$ is smaller or larger than -4 therefore determines the qualitative behavior of the resulting distributions: Figure 15 shows that for $n=4$, all values of $\lg \rho_{\Lambda}$ between $\rho_{\Lambda}^{\min }$ and $\rho_{\Lambda}^{\max }$ are roughly equally likely, whereas smaller or larger $n$ cause the distributions to be peaked at $\rho_{\Lambda}^{\min }$ and $\rho_{\Lambda}^{\max }$, respectively. There are thus only three classes of theories that are not ruled out by the observed $\rho_{\Lambda}$-value (shaded band):

1. Theories where $n \sim-4$.

2. Theories where inflation predicts a very narrow distribution $f^{\text {inf }}(Q)$ centered around or slightly below the observed $Q$-value.

3. Theories where the weight function $w(Q)$ is sufficiently sharply peaked around the observed $Q$-value to overpower the pull towards $\rho_{\Lambda}^{\min }$ or $\rho_{\Lambda}^{\max }$.

None of these classes are problem free. Class 1 involves inflationary fine-tuning in the sense that it requires the sharp inflationary prediction for $\lg Q$ to coincidentally fall within the range around -5 where $w_{Q}(Q)$ is substantial. We argued in Section VIC 3 that $w_{Q}(Q)$ may be rather broad, suggesting that models in class 3 will only work if $n$ is not too far from -4 . We saw in Section $\mathrm{V}$ that inflation models with a "natural" horizontal energy scale $m_{h} \lesssim \bar{m}$ do not fall into class 1 , but rather that their raw predicted $Q$-values display a high $Q$ tail with $n \approx-1$ (heavy curve in Figure 15). Such models thus tend to overpredict $\rho_{\Lambda}$ by orders of magnitude, which is yet another manifestation of the smoothness problem discussed in Section VIF.

Figure 16 is an attempt to illustrate the smoothness problem and this intimate relation between $\rho_{\Lambda}$ and $Q$. In summary, we have found no compelling solution to the smoothness problem. High energy inflation models with $m_{h} \gg m_{\mathrm{Pl}}$ can have $m_{h}$ fine-tuned to fall into class 1 , but may flounder on quantum gravity corrections. Low energy inflation models with $m_{h} \lesssim m_{\mathrm{Pl}}$, on the other hand, predict $\left(\rho_{\Lambda}, Q\right)$ near the five-pointed star in Figure 16, quite far from the observed value. If a future candidate inflation model provides a compelling solution to the smoothness problem, this will thus be a non-trivial and noteworthy achievement. 


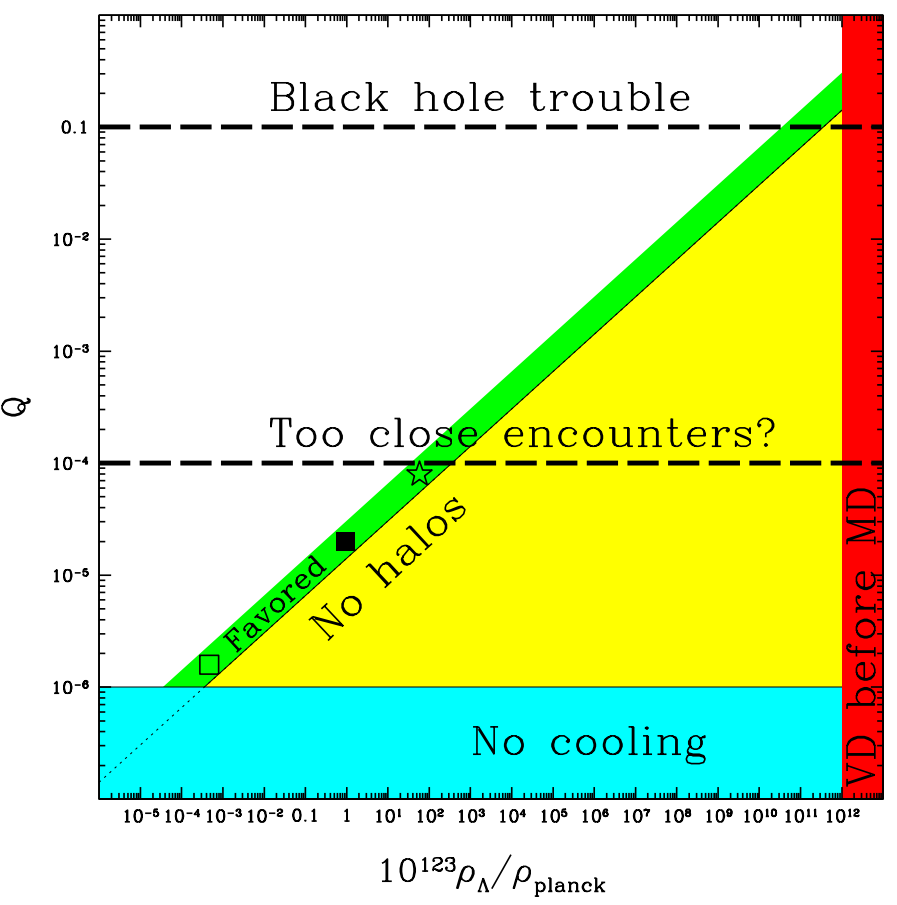

FIG. 16: The weight function $w(\mathbf{p})$ (the number of reference objects produced per unit thermalized volume) causes important modulations of the raw inflationary predictions in the $\left(\rho_{\Lambda}, Q\right)$ plane, with $w_{\text {halo }}(\mathbf{p})$ giving essentially no weight to the region marked "no halos" and $w(Q)$ strongly downweighting regions more than about an order of magnitude above or below $Q=10^{-5}$. The fact that inflation typically predicts $\rho_{\Lambda}$ to have a uniform distribution in this range, uncorrelated with $Q$, gives probability $\propto \rho_{\Lambda}$ in this plane, so nearly all probability lies within the diagonal green/grey favored band near the halo limit. The logarithmic slope of the inflationary Q-distribution determines the most likely outcome, with $d \ln f_{Q}^{\text {inf }} / d \ln Q \ll-4$ giving the open square and $d \ln f_{Q}^{\text {inf }} / d \ln Q \gg-4$ giving the star. The filled square shows the measurements from Table 1.

\section{G. Calculation summary}

Let us summarize the recipe that we have derived for computing the cosmological parameter distribution $f_{p}(\mathbf{p})$.

1. Use one of the two Monte Carlo approaches of Section $\mathrm{V}$ (depending on whether you wish to exclude finite pockets or not) to generate a large number of parameter vectors $\mathbf{p}_{i}$ drawn from the raw inflationary distribution $f_{p}^{\text {inf }}(\mathbf{p})$, keeping only those with $N_{\text {tot }}>N_{\text {gal }} \approx 55$.

2. To approximate the $\rho_{\Lambda} \rightarrow 0$ limit of equation (79), discard all vectors with $\rho_{\Lambda}<0$ or $\rho_{\Lambda}>\rho_{\Lambda}^{*}$, reducing $\rho_{\Lambda}^{*}$ until the results stop changing.

3. Compute $f_{\Lambda}\left(\rho_{\Lambda}\right)$ using equation (82).

4. Compute the distributions for all other parameters as $f_{p}(p) \propto w_{Q}(Q) Q^{3} f_{p}^{\text {inf }}(\mathbf{p})_{\mid \rho_{\Lambda}=0}$ by weighting the points from Step 2 by $w_{Q}\left(Q_{i}\right) Q_{i}^{3}$ and then making histograms.

In practice, Step 4 can be done either by unequal point weights $w_{Q}\left(Q_{i}\right) Q_{i}^{3}$ in the histograms or by resampling, drawing a large number of points from Step 2 at random with probability $\propto w_{Q}\left(Q_{i}\right) Q_{i}^{3}$. We use the latter approach. Figure 1 shows a complete worked example for the case of Measure A, $m_{h}=10 m_{\mathrm{Pl}}$ and $m_{v}=0.002 m_{\mathrm{Pl}}$.

\section{ANALYTIC RESULTS}

In the two preceding sections, we computed cosmological parameter predictions for specific potentials and measures, finding that unless $m_{h} \sim m_{\mathrm{Pl}}$, the dependence on the inflaton potential could be understood analytically in terms of its peak and trough statistics. We did this with pocket-based orderings, so to better understand how the choice of measure affects the predictions, let us now revisit this computation using the other class of orderings from Section IV: global orderings. We will find that simple analytic calculations allow us to rule out a broad class of global orderings, notably many that give attractor behavior and make the predictions independent of pre-inflationary initial conditions.

In Section IV, we saw that a global ordering is uniquely defined by specifying a "time" variable to order the reference objects by. We found that all observable quantities (e.g., $\rho, T$ or $H$ ) are inadequate as such global time variables, leading instead to pocket-based orderings, so let us now explore time variables that are not observable (except in differences or ratios, like $t$ and $a$ ).

We will focus most of our discussion on the case of ordering by time $t^{13}$, then discuss how these conclusions can be generalized to other global orderings. This naturalsounding choice is one of the first to have been explored in detail, and was used in some of the key early work on eternal inflation $[8,53,62,103-106]$.

13 Specifically, we define $t$ as the time variable in synchronous coordinates where the metric is

$$
d s^{2}=d t^{2}-a(\mathbf{x}, t)^{2} d \mathbf{x}^{2} .
$$

The lines of constant $\mathbf{x}$ in this metric are timelike geodesics corresponding to the worldlines of Gedanken co-moving observers, and $t$ is the proper time of these observers. This coordinate system remains well-defined until these geodesics start to cross, which happens only long after thermalization when fluctuations go non-linear — the coolness problem discussed below becomes severe long before then, so it cannot be circumvented by a creative choice of gauge at late times. When we discuss $t$ for a particle in the present epoch, the rigorously inclined reader can simply take this to mean its proper time, since this provides a well-defined ordering even after geodesic crossing. 


\section{A. The origin of attractor behavior}

One of the most appealing features of $t$-ordering is that it generically gives attractor behavior, allowing a unique parameter probability distribution $f_{p}(\mathbf{p})$ to be computed independently of the initial conditions. This is most elegantly seen using the Fokker-Planck equation formalism, giving the time-evolution of $V_{\phi}(\phi)$, the physical volume associated with different $\phi$-values. Thorough and detailed treatments of this are given in, e.g., $[8,53,62,103-106]$, so we will only summarize the results here and attempt to provide physical intuition for them. The Fokker-Planck equation takes the form

$$
\dot{V}_{\phi}(\phi)=L V_{\phi}(\phi)
$$

where $L$ is a linear operator involving $0^{\text {th }}, 1^{\text {st }}$ and $2^{\text {nd }}$ derivatives with respect to the inflaton field $\phi$ to incorporate the effects of expansion, slow-roll and quantum diffusion, respectively. The total volume at a given time is thus

$$
V(t) \equiv \int V_{\phi}(\phi, t) d^{d} \phi
$$

which is conveniently partitioned as the sum of two contributions $V_{\text {infl }}(t)$ and $V_{\text {therm }}(t)$ corresponding to integrating over the parts of $\boldsymbol{\phi}$-space where space is inflating and has thermalized, respectively. (The standard prescription is to reclassify a volume of space from inflating to thermalized as soon as its $\phi$-value leaves the part of $\phi$-space where the slow-roll approximation is valid and to not evolve it further $[8,53,62,103-106]$, so that $V_{\text {therm }}(t)$ is simply the volume of the U-shaped hypersurfaces in Figure 4 that lie in the part of spacetime with time coordinate $\leq t$.)

If the total volume $V(t)$ is finite at some initial time $t$, then both $V_{\text {infl }}(t)$ and $V_{\text {therm }}(t)$ will remain finite for all $t$ since the expansion rate $H$ cannot be infinite. Inflation is usually said to be eternal ${ }^{14}$ if $V_{\text {infl }}(t) \rightarrow \infty$ as $t \rightarrow \infty$. If the number of reference objects is proportional to the thermalized volume produced, i.e., $V_{\text {therm }}(t)$ (as is the case for points, protons, planets and all other reference objects we have discussed), then inflation produces an infinite number of reference objects provided that $V_{\text {therm }}(t) \rightarrow \infty$ as $t \rightarrow \infty$. Such eternal inflation with infinitely many reference objects occurs quite generically, whenever the inflaton potential $V(\phi)$ contains an "eternal inflation region". It is well known that there are three types of eternal inflation regions [48-50, 108]:

1. A local maximum in $V(\phi)$ where the slow-roll approximation is valid $[62,109]$.

\footnotetext{
14 Throughout this paper, we use eternal to mean eternal to the future. For recent discussions of whether inflation can be eternal to the past, see $[48-50,100,101,107]$ and references therein. For an up-to-date discussion of initial conditions and whether they are needed, see [102].
}

2. A $\phi$-range where $V(\phi)$ is large enough for quantum diffusion to dominate the dynamics [32].

3. A local minimum in $V(\phi)$ from which $\phi$ can eventually tunnel out ("old inflation").

In the first case, the volume rolling off the peak in various directions is more than replenished by the expansion of the volume that has not yet rolled off. In the second case, quantum diffusion up the hill causes faster expansion that more than compensates for the fact that it would have been more likely to roll downward - this happens in $\phi$ regions where the slow-roll approximation predicts $Q \gtrsim$ 1.

If there are multiple eternal inflation regions, the fastest expanding one (the one where $V(\phi)$ is largest) always wins, completely dominating the volume distribution $V_{\phi}(t)$ as $t \rightarrow \infty$. [8, 53,62,103-106]. The reason is that if two volumes are eternally expanding with rates $H_{1}$ and $H_{2}$, and $H_{1}>H_{2}$, then the lower second peak will asymptotically contribute a volume fraction

$$
\frac{V_{2}(t)}{V_{1}(t)}=\frac{V_{2}(0) e^{3 H_{2} t}}{V_{1}(0) e^{3 H_{1} t}}=\frac{V_{2}(0)}{V_{1}(0)} e^{-3\left(H_{1}-H_{2}\right) t} \rightarrow 0
$$

as $t \rightarrow \infty$ whatever the initial volume ratio $V_{2}(0) / V_{1}(0)$ was. $^{15}$

Moreover, the fastest expanding eternal inflation region of all (around $\phi_{*}$, say) will completely dominate the asymptotic $t \rightarrow \infty$ volume distribution regardless of the initial conditions, i.e., for any initial distribution $V_{\phi}(\phi)$. This is because quantum diffusion will populate all $\phi$-values and even an exponentially tiny contribution $V_{\phi}\left(\phi_{*}\right)$ created, say, by exponentially suppressed uphill diffusion/tunneling at low density, will eventually overpower all other slower expanding regions as $t \rightarrow \infty$ just as described above. As a result, the asymptotic distribution $V_{\phi}(\phi) / V(t)$ will be determined simply by how long time it takes to roll/diffuse down from $\phi_{*}$ to $\phi$. Since we are evaluating the distribution at fixed time, faster is better. If a volume of space has the inflaton descending from $\phi_{*}$ along a trajectory $\phi(t)$ during the time interval $t_{1} \leq t \leq t_{2}$, then it will expand by $\int_{t_{1}}^{t_{2}} H(\phi(t)) d t$ e-foldings while the eternal region expanded more, by $\left(t_{2}-t_{1}\right) H\left(\phi_{*}\right)$ e-foldings $\left(H(\phi)=\bar{m}^{-1} \sqrt{V(\phi) / 3}\right)$, so the asymptotic probability distribution is $[8,53,62,103-106]$

$$
V_{\phi}(\phi)=V_{\phi}\left(\phi_{*}\right) \exp \left[-3 \int_{t_{1}}^{t_{2}}\left[H\left(\phi_{*}\right)-H(\phi(t))\right] d t\right] .
$$

Since this asymptotic distribution satisfies $\dot{V}_{\phi}(\phi)=$ $3 H_{*} V_{\phi}(\phi)$, it can be computed directly from the FokkerPlanck equation (85) as the solution to the eigenvalue

\footnotetext{
15 Measure ambiguities are thus linked to the fact that we cannot reverse the order of the two limits $n \rightarrow \infty$ in equation (26) and $t \rightarrow \infty$.
} 
problem $L V_{\phi}=3 H_{*} V_{\phi}$ that has the largest eigenvalue $3 H_{*}$. (If this largest eigenvalue is negative, inflation is not eternal.) This eigenvalue problem can be transformed into a manifestly Hermitean form, and has been shown to reduce to a Schrödinger equation [57].

What we care about empirically is not the probability distribution for $\phi$, but the parameter probability distribution $f_{p}(\mathbf{p})$. The latter is readily extracted from the former by keeping track of how inflation ends, i.e., by how the thermalized volume $V_{\text {therm }}(t)$ is distributed among the different basins of attraction and, for each basin, what the probability distribution is for the direction from which it rolled down towards the minimum. A mathematically equivalent alternative to solving the Fokker-Planck equation (see below) is to evolve the inflaton $\phi$ using a stochastic ordinary differential equation that incorporates the effects of quantum diffusion as a random noise term.

\section{B. What $t$-ordering predicts}

A strength of the $t$-foliation solution to the ordering problem is that it makes a specific and well-defined prediction for the parameter probability distribution $f_{p}(\mathbf{p})$ which, because of the attractor dynamics, is independent on initial conditions and unknown pre-inflationary physics. Let us now discuss what this prediction is.

We saw above that in the $t \rightarrow \infty$ limit, all reference objects except for a set of measure zero are in comoving regions of space where $\phi$ came down from the globally highest peak in the potential, $V\left(\phi_{*}\right)$. There is ambiguity as to what happens if more than one peak has $V$ rising to above the Planck scale, because we lack a theory of quantum gravity, but we will not worry about this issue here in light of the more pressing problems described below. Note that the attractor behavior makes not only the initial conditions irrelevant, but also most of the properties of the inflaton potential $V(\phi)$ - all that matters is the shape of the highest peak and its immediate surroundings.

Because of equation (88), the fastest way to come down from this peak is the most favored, and it has been shown [56] that the exponential preference for speed is so strong that the dominant descent mechanism can in some cases be quantum diffusion/tunneling rather than slow roll, giving the strange appearance that we are living in the center of a spherical void (at least for Planckscale inflation). This preference for rapid descent can cause $\phi$ to be quite near the top of the peak 55 efoldings before inflation, so that the tensor amplitude $Q_{t} \sim V\left(\phi_{*}\right)^{1 / 2} / \bar{m}^{2}$. This suggests that a generic inflaton potential with many random-looking peaks will pre$\operatorname{dict} Q_{t} \gg 10^{-5}$ and hence be ruled out by observation unless $V(\phi) \ll 10^{-10} \bar{m}^{4} \sim 10^{9} \mathrm{GeV}^{4}$ for all $\boldsymbol{\phi}$. For instance, the Gaussian random field potentials we explored in Section $\mathrm{V}$ are guaranteed to contain arbitrarily high peaks (exponentially rarely, but this is irrelevant), and are hence all ruled out if $t$-ordering is used. It should be emphasized, however, that there are infinitely many potentials that do not overpredict the fluctuation level with time ordering, most obviously popular single-basin ones like $V(\phi)=m^{2} \phi^{2}$ with $m \approx 5 \times 10^{-6} \bar{m}$.

\section{The coolness problem}

We will now present an argument for why the $t$ foliation solution to the ordering problem is observationally ruled out. This is closely related to arguments given in $[48-50,56]$ as described below.

Consider any reference objects the number of which is proportional to physical thermalized volume, say protons, planets or people. Let us compute the probability distribution $f_{T}(T)$ for the CMB temperature $T$ observed at such reference objects at a given time $t$. We will find that our universe is much cooler than predicted.

After a given volume thermalizes at time $t_{\text {therm }}$, it keeps expanding and its photon temperature $T_{\mathrm{CMB}}$ drops. We can therefore write $T_{\mathrm{CMB}}=f\left(t-t_{\text {therm }}\right)$ for a known and monotonically decreasing function $f$. In Planck units $\left(m_{\mathrm{Pl}}=1\right.$; used throughout this section $)$, we have

$$
f(\Delta t) \sim \begin{cases}\Delta t^{-1 / 2} & \text { during radiation domination, } \\ \xi^{-1 / 3} \Delta t^{-2 / 3} & \text { during matter domination, }\end{cases}
$$

where $\xi \approx 3.3 \times 10^{-28}$ is the matter-to-photon ratio from Table 1. ( $T_{\mathrm{CMB}} \propto a^{-1}$ at all times.) Since the number of refence objects is proportional to the thermalized volume, the fraction of them in domains thermalized by time $t$ is

$$
P\left(t_{\text {therm }}<t\right) \propto V_{\text {therm }}(t) \propto e^{3 H_{*} t} .
$$

The fraction of the reference objects where $T_{\mathrm{CMB}}$ is below some given value $T$ is therefore

$$
\begin{aligned}
P\left[T_{\mathrm{CMB}}<T\right] & =P\left[f\left(t-t_{\text {therm }}\right)<T\right] \\
& =P\left[t_{\text {therm }}<t-f^{-1}(T)\right] \\
& \propto e^{3 H_{*}\left[t-f^{-1}(T)\right]} \\
& \propto e^{-3 H_{*} f^{-1}(T)}
\end{aligned}
$$

where the inverse function $\Delta t=f^{-1}(T)$ is approximately

$$
f^{-1}(T) \sim \begin{cases}T^{-2} & \text { during radiation domination, } \\ \xi^{-1 / 2} T^{-3 / 2} & \text { during matter domination, }\end{cases}
$$

Since the expression $e^{-3 H_{*} f^{-1}(T)}$ in equation (90) is a perfectly regular and well-behaved function, approaching zero as $T \rightarrow 0(t \rightarrow \infty)$ and approaching unity as $T \rightarrow$ $T_{\text {therm }}$, eternal inflation with $t$-ordering thus makes the firm testable prediction that the temperature probability distribution is given by $P\left[T_{\mathrm{CMB}}<T\right]=e^{-3 H_{*} f^{-1}(T)}$. During matter domination, we thus have the prediction

$$
P\left(T_{\mathrm{CMB}}<T\right) \approx e^{-\left(\frac{T}{T_{*}}\right)^{-3 / 2}},
$$


where $T_{*} \equiv \xi^{-1 / 3}(3 H)_{*}^{2 / 3} \approx 10^{9} H_{*}^{2 / 3}$. For definiteness, let us take the eternal inflation expansion rate to be a typical inflationary rate $H_{*} \sim 10^{-5} / 10^{-38} \mathrm{~s}$, which gives $T_{*} \sim 10^{38} \mathrm{~K}$ - the conclusions we will draw are completely unaffected by varying $H_{*}$ within the observationally allowed range. This gives the spectacularly small probability

$$
P\left(T_{\mathrm{CMB}}<3 \mathrm{~K}\right) \approx 10^{-10^{56}},
$$

and the probability of finding $T_{\mathrm{CMB}}$ as low as observed by COBE/FIRAS, i.e., $T_{\mathrm{CMB}}=2.728 \pm 0.004$, is of course even smaller. Even if we take as a given that $T_{\mathrm{CMB}}<4 \mathrm{~K}$, this probability remains minuscule: $P\left(T_{\mathrm{CMB}}<3 \mathrm{~K} \mid T_{\mathrm{CMB}}<4 \mathrm{~K}\right) \approx 10^{-10^{55.6}}$. Indeed, the probability is small even of having the CMB temperature a mere $10^{-17} \mathrm{~K}$ warmer, corresponding to a one second younger (relative to the thermalization time) universe: about $e^{-(1 \mathrm{sec}) H_{*}} \approx 10^{-10^{38}}$. Although it is easy to envision an anthropic weight factor $w(\mathbf{p})$ that may slightly skew this distribution towards lower temperatures (by requiring sufficient time for evolution, say), such corrections will be clearly be way too week to overpower this double exponential preference for high temperature indeed, before CMB temperature was measured in 1965, scientists saw no fundamental problem with it being say $5 \mathrm{~K}$ as predicted by Alpher \& Herman [110]. The FIRAS measurement of the CMB temperature therefore rules out eternal inflation with time ordering at a significance level of about $99.999 \ldots 9 \%$, where the dots denote $10^{56}$ nines. Few scientific theories have had the honor of being ruled out with greater statistical significance.

The root of the failure is clearly that this ordering rewards rapid expansion, so that at a fixed time, the vast majority of all reference objects formed only very recently. Although we focused on $T_{\mathrm{CMB}}$ above to be specific, the coolness problem clearly afflicts many other observable parameters as well. Our universe is not only cooler than predicted, but also older and less dense than $t$-ordering predicts. These paradoxical implications have been discussed in the literature, although in contexts leading to somewhat less negative conclusions. This issue is at the core of the surprising findings of Linde \& Mezhluminan [56] that we might expect to find ourselves in the center of a rare spherical void, and the authors mention this as a perhaps a possible way to obtaining an open $\Omega_{\text {tot }}<1$ universe from inflation. Guth [48-50] discusses the problem in detail, terming it the "youngness paradox". He speculates that this could explain why the SETI project has failed to discover extraterrestrial intelligence (since the preference for low cosmic age implies that we are overwhelmingly likely to be the first observers in our Hubble volume), but finds it more plausible that it is merely a symptom of the probability calculation technique not being the right one. A measure with $t$-ordering implies not only this, but also more extreme consequences such as that you personally are the first observer in your Hubble volume since thermalization, and that it is way more likely that you evolved on the first habitable planet to form, under circumstances where many apparent flukes accelerated your arrival. We conclude that our living in a cool, leisurely universe means nothing other than that $t$-ordering is the wrong ordering.

\section{Why does not rule out eternal inflation but limits attractor behavior}

There is presumably some correct solution to the ordering problem, and we have found that $t$-ordering is not the one. It is tempting to speculate that this is because it flaunts some important yet-to-be-established underlying physical principle, in which case the coolness problem is a helpful clue for identifying this principle. Here is a guess as to such a principle: Physical questions must be expressible in terms of observables. This implies that it is a big no-no to condition on quantities that are not physically observable, like $t$ or $a$. $t$-ordering violates this principle since, as described above, it corresponds to defining reference objects such as, say, "protons at time $t$ ". The global time $t$ is a completely unobservable quantity, because all we can measure in our thermalized region is time intervals, e.g., the time interval $\left(t-t_{\text {therm }}\right)$ since inflation ended here. Moreover, there are infinitely many alternative choices of time variable, and between causally disconnected regions of spacetime (such as two different thermalized pockets), gauge ambiguities imply that there is no objective way of defining simultaneity.

Let us now discuss the implications of the coolness problem for eternal inflation and attractor behavior. Since the same Fokker-Planck probability approach that elegantly predicts eternal inflation is ruled out by the coolness problem, is inflation really eternal in any meaningful sense? A standard definition of eternal inflation (e.g.,[57]) is that "at any given time, part of space is inflating, and the inflating volume increases over time", yet this definition uses precisely the infamous time foliation by considering the situation "at any given time".

Inflation can nonetheless be eternal, in the following well-defined sense: A finite comoving volume can produce an infinite physical volume and an infinite number of particles (and other reference objects). Equivalently, inflation can be eternal in the sense of producing infinite Level I multiverses according to the classification of [51]. This is readily seen in the following simple example, which is illustrated in Figure 4. Consider a single-field inflaton potential $V(\phi)$ with a peak (local maximum) at $\phi=0$, and a comoving spatial region where the inflaton potential varies extremely slowly in the $x$-direction:

$$
\phi(x, y, z) \approx \varepsilon x,
$$

where the constant $\varepsilon$ is so small that spatial gradients in $\phi$ are negligible. If quantum diffusion is negligible, then inflation will eventually end everywhere in our comoving region except at the one point $\phi=0$, but it clearly takes longer for $\phi$ to roll off the peak and end inflation the 
closer to $\phi=0$ it starts. In the slow-roll approximation and considering the very top of the peak where it can be approximated by an upside down parabola, one readily finds that the number of $e$-foldings required to roll down from $\phi$ is given by (Appendix B.2)

$$
e^{N(\phi)} \propto|\phi|^{\eta^{-1}}
$$

where $\eta \equiv \bar{m}^{2} V^{\prime \prime}(0) / V(0)$ is the usual slow-roll parameter at the origin. The total thermalized volume produced by our comoving region is thus

$$
V \propto \int e^{3 N(\phi(\mathbf{r}))} d V \propto \int e^{3 N(|\varepsilon x|)} d x \propto \int|x|^{3 \eta^{-1}} d x,
$$

which will diverge near the origin and give an infinite volume as long as $\eta>-3$. This simply reexpresses the wellknown result that slow-roll peaks are eternal inflation regions, but without involving the Fokker-Planck equation or $t$-foliation. An alternative way of seeing this is to compute the probability distributions in equation (56). If the initial conditions give a uniform distribution for $\phi$ around the peak, then since $\eta<0$, equation (94) readily gives well-behaved probability distributions $f^{\text {inf }}$ for $N_{\text {tot }}$, $V \propto e^{3 N_{\text {tot }}}, a \propto e_{\text {tot }}^{N}$ and $t \approx N_{\text {tot }} / H_{0}$ at thermalization, where $H_{0} \equiv \bar{m}^{-1} \sqrt{V(0) / 3}$ :

$$
\begin{aligned}
P\left(N_{\mathrm{tot}}>N_{*}\right) & =e^{\eta N_{*}}, \\
P\left(V>V_{*}\right) & \propto V_{*}^{\eta / 3}, \\
P\left(a>a_{*}\right) & \propto a_{*}^{\eta}, \\
P\left(t>t_{*}\right) & \approx e^{\eta H_{0} t_{*}} .
\end{aligned}
$$

Multiplying the corresponding differential probability distributions $f^{\text {inf }}$ by the volume factor $w_{\text {vol }} \propto e^{3 N_{\text {tot }}}$ in equation (56) gives the predicted probability distribution $f_{p}$ for the parameters of a random thermalized volume element:

$$
\begin{aligned}
P\left(N_{\text {tot }}<N_{*}\right) & \propto e^{(3+\eta) N_{*}}, \\
P\left(V<V_{*}\right) & \propto V_{*}^{1+\eta / 3}, \\
P\left(a<a_{*}\right) & \propto a_{*}^{3+\eta}, \\
P\left(t<t_{*}\right) & \propto e^{(3+\eta) H_{0} t_{*}} .
\end{aligned}
$$

Since $\eta \geq-1$, these distributions are all unnormalizable, so the probability is zero that $N_{\text {tot }}, V, a$ or $T$ is finite.

The second type of eternal inflation region (the kind dominated by quantum diffusion) also survives even though the standard justification for it becomes invalid. The standard argument [32] is that quantum diffusion up the hill causes faster expansion that more than compensates for the fact that it would have been more likely to roll downward, but it is of course precisely such measures favoring faster expansion that lead to the coolness problem. An alternative justification is to again consider the evolution of a single comoving spatial region, without applying any volume weighting until after thermalization. When quantum diffusion is included, $\phi(t)$ will be not the deterministic slow-roll trajectory, but a random walk. This is guaranteed to end after some finite time $t_{\text {end }}$ when $\phi$ leaves the slow-roll region of $\phi$-space, after which the comoving region thermalizes with a volume

$$
V \propto e^{3 N_{\text {tot }}}, \quad N_{\text {tot }} \equiv \int_{0}^{t_{\text {end }}} H(\phi(t)) d t .
$$

Although both $N$ and $V$ are clearly finite for any trajectory $\phi(t)$, the volume expectation value $\langle V\rangle$ can easily be infinite. As a simple example, if the random variable $N_{\text {tot }}$ has an exponential distribution $f_{N}\left(N_{\text {tot }}\right) \propto e^{-N_{\text {tot }} / \bar{N}}$ for some constant $\bar{N}$ (equation (96) is such an exponential distribution with $\bar{N}=-\eta^{-1} \geq 1$ ), then just as in the slow-roll peak case above, the probability that $N_{\text {tot }}$ is less than any finite value vanishes if $\bar{N}>1 / 3$. Such an ensemble averaging is equivalent to what happens when averaging (integrating) over a finite comoving volume as in equation (95), since the quantum fluctuations are effectively uncorrelated in causally disconnected patches, and infinitely many such patches develop. In other words, both of the "new inflation" mechanisms that can generate eternal inflation as per the $t$-foliated Fokker-Planck formalism can do so also in the sense of producing infinitely many reference objects in a finite comoving region.

A different way of seeing this is to note that the thermalization surfaces are perfectly well-defined physical hypersurfaces in spacetime, so there are no ambiguities regarding the question of whether they have finite or infinite volume. Therefore the Fokker-Planck approach to computing this volume must give the same answer as the differential equation approach described above indeed, equation (89) agrees with equation (103) with $H_{*}=(1+\eta / 3) H_{0}$. Ambiguities only arise if we ask whether one infinite volume is larger than another infinite volume.

Although eternal inflation thus survives the coolness problem unscathed, the attractor dynamics giving testable prediction $f_{p}(\mathbf{p})$ independent of the initial conditions is severely challenged. There is of course nothing wrong with the Fokker-Planck equation per se: it can be consistently reexpressed in terms of any time variable one choses $[8,53,62,103-106]$, so the problem lies entirely with the limiting procedure used to extracting probabilities from it. However, modulo a tunneling caveat discussed below in Section VII G, the Fokker-Planck equation only exhibits this attractor dynamics when expressed in such a way that it rewards rapid expansion, since as described above, this was the mechanism by which the highest peak in the potential was able to dominate the asymptotic solution. To circumvent the coolness problem, one must therefore avoid rewarding rapid expansion. There are two ways of doing this:

1. Changing the reference objects to be comoving rather than physical volume elements, i.e., replacing the volume weight factor of equation (57) by $w_{\mathrm{vol}}(\mathbf{p})=1$. 
2. Changing the time variable to something not rewarding faster expansion, like $a$ or $\rho$.

Either way, the attractor dynamics described above goes away, and the asymptotic distribution (if it exists) will depend on initial conditions (modulo tunneling issues to which we return in Section VII G). The former approach has been studied in, e.g., [8, 53, 62, 103-106], and can be argued to be unphysical since all reference objects that may be related to observers (particles, planets, etc.) are proportional to physical rather than comoving volume. Let us now consider the latter. Although changing the time variable to $\rho$ or other observable quantities solves the coolness problem, we have seen that this leads to pocket-based orderings which we have already discussed, so let us turn instead to unobservable time variables such as $a$.

\section{E. $a$-ordering and others not favoring rapid expansion}

There are infinitely many possible choices of time variable, and different choices can give different predictions. $[57,58,61]$. As an interesting example giving qualitatively different predictions from $t$-ordering, let us consider ordering by the cosmic scale factor $a$.

First of all, $a$-ordering is not afflicted by the coolness problem. This is readily seem from comparing equation (102) with equation (103) and noting that the exponential divergence is replaced by much milder one that is between quadratic and cubic. Since $T_{\mathrm{CMB}}=$ $T_{\text {therm }} a_{\text {therm }} / a$, repeating the derivation of Section VIF shows that equation (90) gets replaced by

$$
\begin{aligned}
P\left[T_{\mathrm{CMB}}<T\right] & =P\left[T_{\text {therm }} a_{\text {therm }} / a<T\right] \\
& =P\left[a_{\text {therm }}<a T / T_{\text {therm }}\right] \\
& \propto\left(a T / T_{\text {therm }}\right)^{3+\eta} \\
& \propto T^{3+\eta} .
\end{aligned}
$$

The probability of observing $T_{\mathrm{CMB}}<3 \mathrm{~K}$ given than $T_{\mathrm{CMB}}<4 \mathrm{~K}$ therefore has the acceptable value $(3 / 4)^{3+\eta} \approx 0.5$ rather than $10^{-10^{56}}$ as with $t$-ordering.

As elaborated in, e.g., $[57,58,61]$, replacing $t$ by $a$ as a time-variable reverses some qualitative conclusions. With the initial conditions of equation (93), the amount of thermalized volume produced up to a given time is readily found to be

$$
V_{\text {therm }} \propto \frac{e^{(3+\eta) H_{0} t}}{1-3 \eta^{-1}} \approx \frac{a^{3+\eta}}{1-3 \eta^{-1}} .
$$

Since $\eta \geq-1$ at a the peak, the amount of thermalized volume produced thus grows steadily. Over time, the freshly thermalized volume comes from an increasingly tiny initial volume very close to the peak (reflected by the $\eta$ in the exponent), but this negative effect is more than offset by the huge inflationary expansion (giving the 3 in the exponent).
Equation (106) shows that when the inflaton potential has multiple peaks, $t$-ordering and $a$-ordering give radically different predictions. With $a$-ordering, taking $a \rightarrow \infty$ shows that the flattest peak (which has the largest $[3+\eta])$ will completely dominate the thermalized volume, and that peak height $H_{0}$ is irrelevant. With $t$-ordering, on the other hand, taking $t \rightarrow \infty$ shows that the peak with the largest value of $(3+\eta) H_{0}$ will completely dominate the thermalized volume, i.e., that the most important factor is peak height $H_{0}$, modulated by a slight preference for flatness. By constructing new time variables that are functions of $t, a$ and observables like $H$, one can readily construct a range of other predictions.

In conclusion, global orderings based on a nonobservable time variable are not all ruled out by observation, but lack compelling physical motivation and, as a class, fail to make unambiguous predictions. Another argument against global ordering with "time" variables such as $a, T, H$ and $\rho$ is that they can change direction (when a spatial region starts to contract) and hence fail to smoothly cover the entire spacetime manifold. Most importantly, if we accept the observability principle of Section VII D that physical questions must be expressible in terms of observables, then this entire class of orderings is ruled out.

\section{F. Ordering by an observable and the pothole paradox}

Equation (62) shows that the number of reference objects is proportional to the volume at thermalization, so if two initially equally large volumes roll off the same peak in $V(\phi)$ in opposite directions, the one that requires more $e$-foldings to thermalize will produce more reference objects in the end. As we will now see, this has an important implication for pocket-based orderings.

Consider the number of reference objects that will ultimately form in a given comoving volume. Equation (62) shows that it is proportional to the physical volume at thermalization, i.e., that it grows during inflation and then stays constant. This implies that whatever time variable we use, most reference objects at a given "time" are in regions that stopped inflating as recently as allowed by their existence. Above we saw that this effect was rather mild with the time variable $a$ but gave the catastrophic coolness problem with the time variable $t$. When conditioning not on a monotonically increasing time variable but on an observable quantity such as $\rho, T$ or $H$ (implying a pocket-based ordering), the effect is that the longest possible inflation scenario will completely dominate the statistics, no matter how long it takes. This means that a flatter peak dominates over a shallower one. However, it also means that both will be outperformed if the inflaton potential has a pothole in the roll path where $\phi$ will get stuck for an exponentially long time until it tunnels out [80]. The deeper the pothole, the better, so one could argue that almost all 
observers can trace the origin of their pocket back to an exponentially rare tunneling event through a high peak in the potential. This event in turn would be overwhelmingly likely to have been preceded by a many other freak tunneling events. This is a disturbing scenario, since for any extremely long inflationary history there exists an even longer one, and and it is far from clear that it gives well-defined and observationally allowed cosmological parameter predictions $f_{p}(\mathbf{p})$. Note that the exponentially small probability per unit time to tunnel out of a deep inflating minimum is irrelevant, since $\phi$ is guaranteed to tunnel out eventually with probability unity.

The "pothole paradox" above follows from strict adherence to weighting pockets by their number of reference objects, even though each pocket contains infinitely many ${ }^{16}$. In other words, it follows from assuming that some infinities are greater than others. This argues against inter-pocket weightings 3, 4 and 5 from Section IV B 2, suggesting that that all countable infinities receive equal weight.

\section{G. Quantum tunneling and attractor behavior}

One area in need of further work is the effect of quantum tunneling in inflation. In some cases, for example ones involving the above-mentioned pothole paradox, this tunneling process may hold the key to understanding the qualitative predictions.

As a specific example, consider the question of whether $a$-ordering gives attractor behavior or gives predictions that depend on initial conditions. As discussed above, $a$ ordering is equivalent to weighting by comoving volume $V_{\mathrm{c}}$, which is a conserved quantity. This means that, ignoring quantum diffusion and tunneling, the distribution $V_{\mathrm{c}}(\phi, a)$ simply flows down into the valleys, never moving from one basin of attraction to another. In other words, initial conditions matter, since the probability of ending up in a given basin of attraction is simply the probability of starting out in that basin of attraction. Quantum tunneling alters this conclusion, allowing transitions between basins either during inflation or after reheating, in the second inflation phase corresponding to a cosmological constant $\rho_{\Lambda}>0$ [114]. Modeling this as a Markov process with a matrix of jump probabilities per unit "time" $a$ between the different basins, one can imagine convergence to an equilibrium state as $a \rightarrow \infty$ where the basin weighting is independent of initial conditions. Further work should clarify whether such attractor behavior actually occurs. For instance, although "downward" tunneling to lower energy through the Coleman-de Lucia bubble nucleation mechanism is fairly well-understood,

16 Note that global orderings lack this ambiguity, since there are only finitely many reference objects at any finite "time" (say $t$ or a) as compared to an infinite number at finite "time" $\rho, T$, etc. the situation regarding "upward" tunneling is less clear. Also, there will be no tunneling out of a minimum with $V(\phi)=0$ to first order, thereby spoiling attractor behavior if there is more than one such minimum, but it is not clear that there is no tunneling out to second order.

\section{CONCLUSIONS}

If string theory or some other fundamental theory predicts an effective inflaton potential $V(\phi)$, then it will clearly be worthwhile to compute the predicted cosmological parameter probability distribution $f_{p}(\mathbf{p})$ to confront it with observation. In this paper, we have attempted to strengthen the groundwork necessary for such a calculation in the context of classic slow-roll inflation, exploring how the predictions for the 8 parameters $\mathbf{p}=\left(\Omega_{\mathrm{tot}}, \rho_{\Lambda}, w, Q, n_{s}, \alpha, r, n_{t}\right)$ depend on both the inflaton potential and the measure. We found that the results depend on a complex and interesting interplay between the two, except for the very simplest potentials with a single minimum that can only be rolled to in one way. It is a persistent myth that models where ensembles and anthropic constraints enter are untestable and hence unscientific [112]. We have seen that, quite to the contrary, most such models that we have explored in the present paper are predictive enough to be eminently testable indeed, so much so that they have already been ruled out by observational data!

Since we found that the measure problem is currently the weakest link in the calculation of predictions, we will classify models below primarily by their potentials and only secondarily by their measure. This is intended not only to extend the shelf life of our discussion, but also to place the seemingly daunting measure problems in context by clarifying what they do and do not affect.

\section{A. How the inflaton potential affects the cosmological parameters}

The effective cosmological constant $\rho_{\Lambda}$ depends only on which basin of attraction $\phi$ rolls into, since it is determined by the minimum in which $\phi$ ends up. The five power spectral parameters $\left(Q, n_{s}, \alpha, r, n_{t}\right)$ vary within the basin, since they depend on the direction from which $\phi$ rolls to the minimum - for one-dimensional potentials, there are only two possibilities (left and right), so they are determined uniquely by the half-basin (region between a potential maximum and minimum). The curvature pa- 
rameter $\Omega_{\text {tot }}$ and total pocket volume $\propto e^{3 N_{\text {tot }}}$ depend on the starting point and hence crucially on the measure. Finally, the dark energy equation of state $w=-1$ as elaborated below in Section VIII D.

One of our key findings is a useful simplification: in all cases except $m_{h} \sim m_{\mathrm{Pl}}$, the cosmological parameter predictions are determined by extremum statistics alone. In other words, unless the characteristic scale $\Delta \phi$ on which the inflaton potential $V$ varies happens to be close to the Planck scale, the only aspect of $V$ that matters observationally is the statistical distribution of peaks and/or troughs.

The $m_{h} \gg m_{\mathrm{Pl}}$ case predicts that

$$
\begin{aligned}
n_{s} & =1-\frac{r}{4}, \\
\alpha & =-\frac{r^{2}}{32}, \\
n_{t} & =-\frac{r}{8} \\
r & \approx 0.15 \pm 0.02
\end{aligned}
$$

and that the parameters $\left(Q, \rho_{\Lambda}\right)$ are determined by trough statistics, specifically by the joint distribution of $V$ and its curvature at minima. Assuming that $V=0$ is not special in the fundamental theory, all that matters is the curvature probability distribution at minima with $V \approx 0$, given for the one-dimensional case by the distribution for $V^{\prime \prime}(\phi)$ where $V^{\prime}(\phi)=0$ and $V(\phi) \approx 0$.

The $m_{h} \ll m_{\mathrm{Pl}}$ case predicts that

$$
\begin{aligned}
n_{s} & \sim 0 \pm 1, \\
\alpha & \approx 0, \\
n_{t} & \approx 0 \\
r & \approx 0
\end{aligned}
$$

and that the parameters $\left(Q, \rho_{\Lambda}, n_{s}\right)$ are determined by peak statistics - for the one-dimensional case and $V=0$ not special, they depend mainly on the the joint probability distribution for $(V, \eta)$ at the peaks.

This means that aside from $w$ and $\Omega_{\text {tot }}$, the number of precise and testable quantitative predictions is 3 when $m_{h} \ll m_{\mathrm{Pl}}$ and 4 when $m_{h} \gg m_{\mathrm{Pl}}$, dropping to only one $\left(n_{t}=-r / 8\right)$ when $m_{h} \sim m_{\mathrm{Pl}}$. In other words, in the 5 -dimensional parameter space $\left(Q, n_{s}, \alpha, r, n_{t}\right)$, the predictions populate a 2-dimensional hypersurface when $m_{h} \ll m_{\mathrm{Pl}}$, a 4-dimensional region when $m_{h} \sim m_{\mathrm{Pl}}$ and a 1-dimensional curve when $m_{h} \gg m_{\mathrm{Pl}}$. In essence, the $m_{h} \ll m_{\mathrm{Pl}}$ limit simplifies since $\epsilon \rightarrow 0$ and the $m_{h} \gg \bar{m}$ limit simplifies since $\left(n_{s}, \alpha, r\right)$ are all determined by $N$, which is in turn known to about $10 \%$.

\section{B. How the measure affects the cosmological parameters}

Above we saw that except when $m_{h} \sim m_{\mathrm{Pl}}$, the predictions for the parameters $\left(Q, n_{s}, \alpha, r, n_{t}, w, \rho_{\Lambda}\right)$ could all be calculated from extremum statistics alone. In other cosmological examples where extremum statistics enter, such as the BBKS Gaussian-peaks formalism for galaxy clustering and the subsequent peak-patch formalism [113], the relevant extremum statistics can be extracted unambiguously from the statistical properties of the function in question (in these examples, the cosmic density field). In our case, however, these extremum statistics are not necessarily those one would obtain by simply locating the appropriate extrema in $V(\phi)$ and tallying up the distribution. Rather, this "raw" distribution is modulated by the measure to give the above-mentioned extremum statistics that determine the parameter prediction. For example, for a homogeneous Gaussian random field $V(\phi)$, the 4-dimensional vector $\left[V(\phi), V^{\prime}(\phi), V^{\prime \prime}(\phi), V^{\prime \prime \prime}(\phi)\right]$ has a simple multivariate Gaussian distribution that can analytically be conditioned on $V\left(\phi^{\prime}\right)=0$ and whatever else is appropriate, but the measure (both initial conditions, ordering and the choice of reference objects) can multiply the resulting distribution of extremum properties by a factor that radically modifies it. Conversely, it is reassuring to note that modifying the extremum distribution is the only way in which the pesky measure problems we have discussed affect the cosmological parameter distribution unless $m_{h} \sim m_{\mathrm{Pl}}$.

Our simulated Measure A case essentially weighted all peaks/troughs equally (strictly speaking, troughs by the lengths of their basins of attraction and peaks by the lengths of the regions to which they flow), except for the rather straightforward conditioning on reference objects in Section VI. For other measures, the extremum statistics can get highly weighted towards certain peaks/troughs over others, either because of their intrinsic properties (like $t$-ordering favoring height and $a$-ordering favoring flatness) or because of good neighbors (like a deep valley to tunnel from in the $a$-ordering case). We saw that $t$-ordering is an extreme case where only the highest slow-roll peak matters, so that the probability distribution is a $\delta$-function corresponding to the highest peak (if $m_{h} \ll \bar{m}$ ) or the trough that $\phi$ can reach the fastest from the highest peak (if $m_{h} \gg \bar{m}$ ).

This author considers the inflationary measure problem wide-open, so let us merely summarize our limited progress with it. We have argued that global orderings such as $t$-ordering that reward rapid expansion are observationally ruled out by the coolness problem (overpredicting the CMB temperature). If we accept the notion that we may only condition on physically observable quantities (e.g., $\rho$ or $T$ ), then we are lead to pocket-based orderings that are specified by an intra-pocket ordering and an inter-pocket weighting. Radial intra-pocket ordering provides a specific working recipe that may and may not be the correct one. Weighting all spatially infinite pockets equally likewise provides a specific prescription that may and may not be right. It avoids the pothole paradox, but is computationally difficult to apply because of the challenge of calculating the relative 
frequencies with which different basins of attraction are rolled down into when quantum diffusion is taken into account. This computation becomes still more complicated in recycling universe [114] and string landscape scenarios [16-29] where each thermalized region can trigger new inflation leading effectively to a Markov process of jumps between basins of attraction.

Since there appears to be only a small-number of wellmotivated measures and they make quite distinct parameter predictions, it may be possible to settle the measure problem empirically by ruling out all but one of them observationally.

\section{Implications for gravitational waves and spectral indices}

Great worldwide efforts are currently being devoted to measuring CMB polarization in the hope of measuring the primordial gravitational wave amplitude $Q_{t}$ via largescale $B$-modes $[115,116]$. A key theoretical question is therefore what amplitude $Q_{t}$ to expect, since this determines the prospects for upcoming experiments to discover what they are looking for and influences their funding and design. Our results are very encouraging in this regard. Models with $m_{h} \gg m_{\mathrm{Pl}}$ predict $r \approx 0.15$, which (Figure 13) is close to current sensitivities and should be detectable already within five years by Planck + SDSS [85]. Models with $m_{h} \ll m_{\mathrm{Pl}}$ predict unobservably small $r$-values, but appear to agree poorly with existing observations: they fail to explain the near scale invariance $n_{s}=0.98 \pm 0.02$ that is observed $[3,86]$ by giving instead a wide distribution with $-1 \leq n_{s} \leq 1$ and they flounder on the smoothness problem by generally overpredicting $Q$. Finally, models with $m_{h} \sim m_{\mathrm{Pl}}$ typically predict $r$-values that are clearly within reach of a next generation CMB polarization satellite; Figure 13 shows typical values $r \sim 0.03$.

The most imminent observational breakthrough may well be sharpening the current limits spectral index limits $n_{s}=0.98 \pm 0.02$ to exclude the scale-invariant HarrisonZel'dovich case $n_{s}=1$, and this will severely reduce the number of viable inflation models. We have seen that models with $m_{h} \gg m_{\mathrm{Pl}}$ predict $n_{s} \approx 0.96$ whereas models with $m_{h} \ll m_{\mathrm{Pl}}$ predict a broad distribution in the range $-1 \leq n_{s} \leq 1-i$.e., they fail to explain why $n_{s} \approx 1$ and cannot give blue $\left(n_{s}>1\right)$ spectra, the only exception being arguably unphysical measures that do not volume weight, like Measure B.

Our $m_{h} \gtrsim m_{\mathrm{Pl}}$ predictions agree well with other arguments suggesting that classic single-field inflation produces both gravitational waves and departures from scale invariance that will soon be observable $[11,117,118]$.

\section{Implications for dark energy}

We saw that in order for the standard inflationary calculation to be self-consistent (give the correct expansion rate $H$ ), the inflaton potential $V(\phi)$ must be defined to include any $\phi$-independent vacuum energy contributions from other sectors of physics, including any bare cosmological constant term that may be present in the Einstein field equations. The effective cosmological constant that we observe in our Hubble volume is therefore simply the height $\rho_{\Lambda} \equiv V\left(\phi_{0}\right)$ of the potential at the minimum that the inflaton has rolled down to here. Any messy inflaton potential $V(\phi)$ thus naturally predicts $\rho_{\Lambda} \neq 0$ unless all its minima happen to have exactly the same height and this common height happens to be exactly zero.

\section{Vanilla or not?}

If no new physics is added beyond this inflaton potential, a generic prediction is that this dark energy will exhibit "vanilla" properties indistinguishable from a cosmological constant, i.e., $w=-1$, a time-independent density at late times (including the present) and no spatial fluctuations. The reason for this is that the effective inflaton mass scale $m$ is huge by current standards ${ }^{17}$. This means that on a very rapid reheating timescale, $\phi$ will settle down into its minimum and stay there, either promptly recollapsing space if the minimum is substantially below zero or giving $\rho_{\Lambda}(t)=V[\phi(t)]$ constant at late times. The huge mass scale $m$ will not only ensure that the minimum is rapidly attained, but also that there are no observable dark energy fluctuations, since excitations in $\phi$ are too massive to be excited by present-day energies. It is noteworthy that the substantial improvements (most recently [119]) in dark energy measurements since the first discovery $[120,121]$ have not established any departures from "vanilla" [122, 123]. However, improved dark energy observations are clearly crucial, since the fact that messy inflation may already explain $\rho_{\Lambda} \neq 0$ and its rough magnitude does not in any way preclude the existence of additional physics producing non-vanilla dark energy at late times, and there are interesting hints at modest statistical significance (e.g., [124]). As shown in Figure 3, improved dark energy observations also complement $\mathrm{CMB}$ polarization observations by measuring the exact same curve $\rho(a)$ at a vastly lower density.

\footnotetext{
17 If we Taylor expand a 1-dimensional potential around a $V \approx 0$ minimum as $V(\phi) \approx m^{2} \phi^{2} / 2$, then equation (32) gives $m=$ $\sqrt{V^{\prime \prime}} \sim m_{h}^{2} / m_{v}$. For the observationally favored case $m_{h} \gtrsim$ $m_{\mathrm{Pl}}$, equation (38) thus shows that $m \sim Q m_{\mathrm{Pl}} \sim 10^{-5} m_{\mathrm{Pl}} \sim$ $10^{14} \mathrm{GeV}$.
} 


\section{Continuum or discretuum?}

Potentials $V(\phi)$ with infinitely many minima (such as Gaussian random fields as explored in Section V) can give a continuous probability distribution for $\rho_{\Lambda}$. In contrast, the landscape picture emerging from string theory suggests a very large but yet finite number of minima, say $n_{\text {tot }} \sim 10^{300}[16-29]^{18}$ Suppose we find a fundamental theory that has $n_{\mathrm{sm}}$ minima which all reproduce our $S U(3) \times S U(2) \times U(1)$ standard model of particle physics at low energies and differ only in their predictions for $\rho_{\Lambda}$. It has been argued that $n_{\mathrm{sm}} \ll n_{\text {tot }}$, i.e., that only a tiny fraction of the minima reproduce our observed lowenergy physics [125]. Our results show that the key for testable predictions is whether $n_{\mathrm{sm}} \gg \xi^{4} Q^{3} \sim 10^{123}$ or not. If $n_{\mathrm{sm}} \gg 10^{123}$, then there may be so many minima able to produce galaxies that $\rho_{\Lambda}$ will for all practical purposes have a continuous probability distribution such as those plotted in this paper, i.e., the "discretuum" $[16,18,19]$ of $\rho_{\Lambda}$-values may be indistinguishable from a continuum. If $n_{\mathrm{sm}} \lesssim 10^{123}$, on the other hand, then there may be only one or a handful of minima producing galaxies, raising the exciting possibility that a precision measurement of $\rho_{\Lambda}$ would allow us to determine precisely which minimum we are in, predict the remaining decimal places of $\rho_{\Lambda}$ and bring closure to our quest for a fundamental theory of physics.

\section{The smoothness problem}

It is striking that even though many people start foaming at the mouth when encountering anthropic $\rho_{\Lambda^{-}}$ arguments, feeling that they render theories untestable, we have found that requiring inflation models to predict a $\rho_{\Lambda}$-value consistent with observation is in fact a very strong test that rules out broad classes of models. The key reason for this is that, as emphasized in [87, 98], requiring nonlinear structures to form strongly constrains the quantity $\rho_{\Lambda} / Q^{3}$ whereas the selection effects acting on the fluctuation amplitude $Q$ alone (with $\rho_{\Lambda} / Q^{3}$ held fixed) appear to be substantially weaker. The suppression of galaxy formation from increasing $\rho_{\Lambda}$ can therefore be largely offset by increasing $Q$, and we found that the

18 There may indeed be a hierarchy of minima in some sort of generalized potential:

1. Different ways in which extra dimensions can be compactified

2. Different discrete fluxes that stabilize the extra dimensions (this sublevel appears to be where the largest number of choices enter, perhaps $10^{300}$ ).

3. Once these two choices have been made, there may be a handful of different minima in the effective supergravity potential. inflation models we explored typically overpredicted $\rho_{\Lambda}$ by a couple of orders of magnitude or more. This is directly linked to the smoothness problem, where the same coupling between $\rho_{\Lambda}$ and $Q$ multiplies the $Q$-distribution by $Q^{4}$ and overpredicts the CMB fluctuation level for most inflation models. In other words, the standard anthropic explanation of the observed $\rho_{\Lambda}$-value does not appear to work well unless we can solve the smoothness problem.

\section{E. Implications for spatial curvature}

Since almost all reference objects are in pockets of infinite volume, all measures except arguably unphysical ones (that do not weight by reference object and hence do not favor infinite volumes over finite ones) predict that we should observe $\Omega_{\text {tot }} \approx 1 \pm 10^{-5}$. A second implication is that the thermalized pocket that we inhabit is spatially infinite, i.e., that our Hubble volume is but one in an infinite "Level I multiverse" [51] with distant Dopplegängers and other Byzantine implications [51, 126]. The reader finding these implications disturbing can take heart in the fact that these inflation models remain quite falsifiable, with Planck + SDSS forecast to shrink the currently allowed range $\Omega_{\text {tot }}=1.01 \pm 0.02[1,2]$ by a factor of four to $\Delta \Omega_{\text {tot }} \approx 0.005[85]$ and therefore potentially excluding this inflationary prediction.

\section{F. Implications for quantum gravity}

If attractor dynamics makes the cosmological parameter predictions $f_{p}(\mathbf{p})$ independent of pre-inflationary initial conditions, then inflation provides a cosmic censorship that erases all quantitative clues about preceding events at higher energy scales. We have argued that many measures providing such attractor behavior are observationally ruled out by the coolness problem, which suggests that perhaps pre-inflationary conditions do matter. If so, drawing conclusions about these conditions will not be easy, requiring both finding $V(\phi)$ and solving the ordering problem, but this nonetheless offers a glimmer of hope that precision measurements of cosmological parameters may ultimately teach us something about quantum gravity.

\section{G. Outlook}

In conclusion, we have studied the important but difficult problem of how to compute testable cosmological parameter predictions from complicated inflaton potentials. Although we have obtained a number of results summarized above, much work remains on multiple fronts here are a few examples:

1. Although many of our results apply also to the multidimensional case $d>1$, others do not, and 
a detailed study study of $d>1$ would be of great interest - particularly $d=2$ and very large dimensionalities like $d \gtrsim 10^{2}$.

2. A better understanding of "upward" quantum tunneling during inflation should settle the issue of if/when initial conditions matter.

3. As concrete potentials $V(\phi)$ emerge from string theory or other fundamental approaches, it will be of great interest to repeat our calculations of $f_{p}(\mathbf{p})$ for these physically motivated potentials.

4. Being the Achilles heel of the entire endeavor, the ordering problem is in grave need of further work. The urgency of this goes beyond cosmology, afflicting any ensemble theory with distinguishable infinite volumes. ${ }^{19}$

This will be challenging work. However, the avalanche of high-precision cosmological measurements is continuing unabated, so if the goal is to find an inflation model that predicts rather than postdicts the correct answer, then the time to rise to this challenge is now.

Thanks to Andy Albrecht, Anthony Aguirre, Edmund Bertschinger, Ted Bunn, Dick Bond, Alan Guth, Shamit Kachru, Andrei Linde, Burt Ovrut, Martin Rees, Paul Steinhardt, Wati Taylor, Neil Turok, Alex Vilenkin and Matias Zaldarriaga for interesting discussions and helpful comments. Thanks to Jean Levitties for providing the park bench upon which most of this manuscript was written. This work was supported by NASA grant NAG511099, NSF CAREER grant AST-0134999, and fellowships from the David and Lucile Packard Foundation and the Research Corporation.

\footnotetext{
19 For instance, much recent work in the string theory landscape context has involved counting minima and quantifying their statistical properties. However, it is far from clear with what statistical weights these minima should be counted - equally (as done in most papers), by basin of attraction area or by some other measure related to, e.g., tunneling or inflation. Solving the measure problem may thus be required for string theory to qualify as a testable physical theory.
}

\section{APPENDIX A: COMPUTING THE OBSERVABLES FROM $\rho(a)$}

In this appendix, we review how the cosmological observables are derived from the cosmic density history $\rho(a)$ and derive the equations of Section II. This is mainly review material [8-15], presented here to clarify when and how most observables can be derived from $\rho(a)$ alone without reference to $V(\phi)$.

Given the cosmic density history $\rho(a)$, the expansion history $a(t)$ is readily obtained by integrating the Friedman equation

$$
H^{2}=\frac{8 \pi}{3 m_{\mathrm{Pl}}^{2}} \rho=\frac{1}{3 \bar{m}^{2}} \rho,
$$

where the Hubble parameter $H \equiv d \ln a / d t=\dot{a} / a$. Luminosity distances, angular diameter distances, and all other classical cosmological observables similarly follow from $\rho(a)$, typically via integrals involving this function. We will now summarize the calculation of the observables $\rho_{\Lambda}(a), \delta_{H}^{T}(k), \delta_{H}^{T}(k)$ and the eight cosmological parameters from Table 1 .

\section{Horizons and e-foldings}

Since the horizon radius is $H^{-1}$, the comoving horizon size $a / H^{-1}=\dot{a} \propto 1 / a^{2} \rho$ is constant on the dotted lines of slope -2 in Figure 3, increasing towards the upper right. Comoving wavelengths leave the horizon whenever the comoving horizon size increases, i.e., whenever the curve $\rho(a)$ is shallower than these dotted lines (when $d \ln \rho / d \ln a>-2$, i.e., when $\ddot{a}>0$ so that the expansion is accelerating), and enter the horizon when the slope is steeper, $d \ln \rho / d \ln a<-2$. When two points lie on the same dotted diagonal, it therefore means that that the horizon volume at the two epochs is the same comoving spatial region. For instance, the comoving spatial volume corresponding to our observable universe now at the present epoch (filled triangle in Figure 3) was the horizon volume during the inflationary epoch corresponding to the open triangle, and the perturbations that left the horizon then are those that we see entering our horizon now.

Key epochs in Figure 3 are $a_{\text {start }}$ (when inflation began; $a_{\text {start }}=0$ if there was no beginning) $a_{\text {exit }}$ (open triangle, when our current Hubble volume left the horizon), $a_{\mathrm{k}}$ (when a fluctuation of wavenumber $k$ leaves the horizon; open square or circle, say), $a_{\text {end }}$ (star, when inflation ended), $a_{\text {reheat }}$ (cross, when reheating ended), $a_{\text {eq }}$ (filled square, matter-radiation equality) and $a_{0}$ (filled triangle, now). The number of e-foldings before the end of inflation is

$$
N(a) \equiv \ln \left(\frac{a_{\mathrm{end}}}{a}\right) .
$$

Of particular importance is $N_{\text {exit }} \equiv N\left(a_{\text {exit }}\right)$, often referred to as just $N$, which is simply $\ln 10$ times horizontal distance between the open circle and the star in 
Figure 3. The figure illustrates that $N_{\text {exit }}$ depends not only on the behavior of $\rho(a)$ during inflation, but also on when reheating ends. ${ }^{20}$ The strong bounds on this quantity derived in $[36,37]$ can be readily read off geometrically from Figure 3 . We must have $\rho\left(a_{\text {exit }}\right) \lesssim 10^{-12} m_{\mathrm{Pl}}$ to avoid overpredicting gravitational waves and density fluctuations (as detailed below) and presumably need $\rho\left(a_{\text {end }}\right) \gtrsim 1 \mathrm{GeV}^{4}$ for reheating to be able to produce protons (c.f. $[127,128])$. This gives the extreme bounds are $35 \lesssim N_{\text {exit }} \lesssim 85$, whereas most popular inflation models favor the narrower range $N_{\text {exit }} \sim 55 \pm 5[36,37]$.

\section{Spatial curvature}

The quantity $\left|\Omega_{\mathrm{tot}}-1\right|^{-1 / 2}$ is simply the ratio of the curvature radius $(\propto a)$ to the horizon radius $H^{-1}$. The spatial curvature parameter $\Omega_{\text {tot }}$ therefore evolves as

$$
\Omega_{\mathrm{tot}}-1 \propto \frac{1}{(a H)^{2}}=\frac{1}{\dot{a}^{2}} \propto \frac{1}{a^{2} \rho},
$$

i.e., $\Omega_{\mathrm{tot}}$ stays constant on the dotted lines of slope -2 in Figure 3 and increases towards the upper right. This means that $\Omega_{\text {tot }}$ is identical on horizon exit and subsequent horizon entry, so that our currently observed $\Omega_{\text {tot }}$ equals $\Omega_{\text {tot }}\left(a_{\text {exit }}\right)$. We will make the standard assumption that, if inflation had a beginning, things were a mess at that time with fluctuations of order unity on the horizon scale, i.e., $\Omega_{\mathrm{tot}}\left(a_{\text {start }}\right) \sim 1$. The inflationary prediction is therefore

$$
\left|\Omega_{\mathrm{tot}}-1\right| \sim \frac{(a H)_{\mathrm{exit}}^{2}}{(a H)_{\mathrm{start}}^{2}}=\left(\frac{\rho_{\mathrm{exit}}}{\rho_{\mathrm{start}}}\right)^{2} e^{-2 N_{\mathrm{before}}},
$$

where $N_{\text {before }}=\ln \left(a_{\text {start }} / a_{\text {exit }}\right)$ is the number of efoldings of inflation before our comoving Hubble volume exited the horizon (open triangle in Figure 3). In typical slow-roll inflation models like the one plotted in Figure 3, the $\rho(a)$-curve is nearly horizontal and so equation (A3) is totally dominated by the second term, giving simply $\left|\Omega_{\text {tot }}-1\right| \sim e^{-2 N_{\text {before }}}$. To match the current observational constraint $\left|\Omega_{\mathrm{tot}}-1\right|<0.02[1,2]$ therefore requires $N_{\text {before }} \gtrsim 2.3$. If the true curvature were exactly zero, density fluctuations on the horizon scale would propagate into our measurement and give $\left|\Omega_{\text {tot }}-1\right| \sim Q \sim 10^{-5}$, corresponding to $N_{\text {before }} \approx 5.8$, so the prediction for the observed curvature is

$$
\left|\Omega_{\text {tot }}-1\right|_{\text {observed }} \sim 10^{-5}+e^{-2 N_{\text {before }}},
$$

20 As discussed in [36], if $\phi$ oscillates around a generic (parabolashaped) minimum in the potential during reheating, the logarithmic slope $d \ln \rho / d \ln a=-3$ just like during matter domination, so ending reheating at higher density increases $N_{\text {exit. }}$. If the second derivative of the potential vanishes in the oscillation direction(s), however, as for $V(\phi) \propto \phi^{4}$, then $d \ln \rho / d \ln a=-4$ like during radiation domination and $N_{\text {exit }}$ becomes independent of when reheating ends.

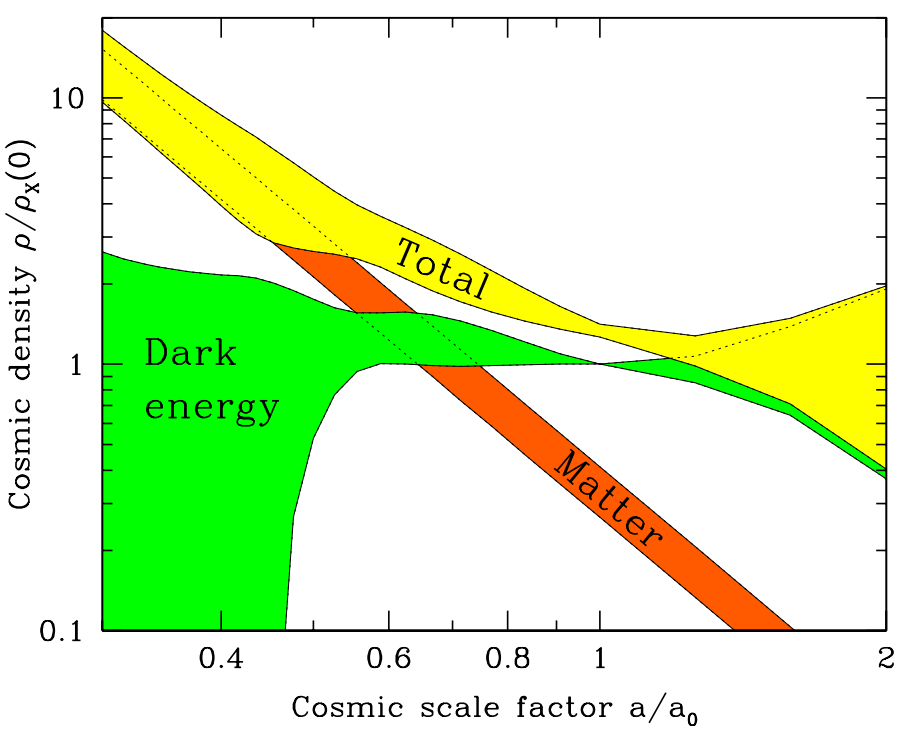

FIG. 17: Zoom of the lower right corner of Figure 3: $1 \sigma$ constraints on the densities of matter and dark energy computed from SN Ia [119], CMB and galaxy clustering data as described in [122].

and $N_{\text {before-values in the range } 6 \lesssim N_{\text {before }}<\infty \text { are }}$ observationally indistinguishable.

\section{Dark energy}

The dark energy density is, in practice, defined as the part of the observed density that we do not understand. The density contributions that we do understand at least at some level are those from photons, matter and curvature, $\rho_{\gamma}, \rho_{\mathrm{m}}$ and $\rho_{\mathrm{k}}$ :

$$
\begin{aligned}
& \rho_{\gamma}(a)=\frac{\pi^{2}}{15} T^{4}=\rho_{\gamma}\left(a_{0}\right)\left(\frac{a_{0}}{a}\right)^{4}, \\
& \rho_{\mathrm{m}}(a)=\frac{2 \zeta(3)}{\pi^{2}}\left(\xi_{\mathrm{b}}+\xi_{\mathrm{c}}+\xi_{\nu}\right) T^{3}=\rho_{\mathrm{m}}\left(a_{0}\right)\left(\frac{a_{0}}{a}\right)^{3}( \\
& \rho_{\mathrm{k}}(a)=3 \bar{m}^{2} H^{2}\left(1-\Omega_{\mathrm{tot}}\right)=\rho_{\mathrm{k}}\left(a_{0}\right)\left(\frac{a_{0}}{a}\right)^{2},
\end{aligned}
$$

where $T=\frac{a}{a_{0}} T_{0}$ is the CMB temperature and $\zeta(3) \approx$ 1.202. The dark energy density is thus the residual

$$
\rho_{\Lambda}(a) \equiv \rho(a)-\rho_{\gamma \mathrm{mk}}(a),
$$

where $\rho_{\gamma \mathrm{mk}}=\rho_{\gamma}+\rho_{\mathrm{m}}+\rho_{\mathrm{k}}$. Since all parameters entering into $\rho_{\gamma \mathrm{mk}}(a)$ are now fairly well measured $\left(\Omega_{\mathrm{tot}}, \xi_{\mathrm{b}}\right.$, $\xi_{\mathrm{c}}, \xi_{\nu}$ as in Table $\left.1, H_{0}, T_{0}\right)$ [1-3], equation (A9) determines $\rho_{\Lambda}(a)$ directly from the behavior of $\rho(a)$ around the present epoch. If $\phi$ settles into a stable potential 
minimum at $\phi_{0}$ long before the present epoch, then we observe $\rho_{\Lambda}=V\left(\phi_{0}\right)$ independently of time, so $w=-1$.

Dark energy is often discussed in the literature as something separate from inflation, so our merging of the two in this paper requires an explanation.

First of all, note that if General Relativity has a true cosmological constant $\rho_{\Lambda}^{\mathrm{GR}}$ hard-wired in, quite separate from the inflaton-only potential $\tilde{V}(\phi)$, then we will only obtain the correct predictions from inflation if we absorb this cosmological constant into the potential and insert $V(\phi) \equiv \tilde{V}(\phi)+\rho_{\Lambda}$ into the equations below. Inserting $\tilde{V}(\phi)$ would give the wrong answer, since the Hubble parameter plays a key roll during inflation and depends on the total density via equation (A1), including $\rho_{\Lambda}^{\mathrm{GR}}$.

Second, note that if there is some other mechanism generating a "dark energy" density contribution without having any connection to the physics of inflation, it must be absorbed into $V(\phi)$ for the same reason. Moreover, for scalar-field models of dark energy, at least ones with standard kinetic terms, it is mathematically straightforward to perform this inclusion, either by modifying the dynamics of the inflaton $\phi$ at very low energies or by including the dark energy fields as additional components in the vector $\phi$.

Third, generic potentials can have many minima, typically with $V(\phi) \neq 0$, so they can readily produce nonzero dark energy regardless of whether there is also a noninflationary source of dark energy. If inflation is related to the string landscape, say, then inflation-based dark energy may not be optional: if there are say $10^{300}$ minima $[18-29,31]$ and $\gg \xi^{-4} Q^{-3} \sim 10^{123}$ of them reproduce our low-energy physics, then it is likely that many minima have $\rho_{\Lambda}$-values in the range $\left|\rho_{\Lambda}\right| \lesssim \xi^{4} Q^{3}$ permitting galaxy formation.

\section{Gravitational waves}

During inflation, quantum fluctuations in any massless field $\psi$ will produce effectively classical fluctuations with power spectrum $P_{\psi}(k)=(H / 2 \pi)^{2}$ at the epoch $a_{k}$ when the scale $k$ exits the horizon [11]. The modes of the spacetime metric corresponding to gravitational waves are examples of such massless fields, and therefore produce current gravitational wave fluctuations [11]

$$
\delta_{H}^{T}(k)=\sqrt{\frac{8 \rho\left(a_{k}\right)}{75 \pi^{2} \bar{m}^{4}}} .
$$

Note that this has nothing to do with fluctuations in the inflaton field, depending on the cosmic density history $\rho(a)$ alone.

The exit scale $a_{k}$ is computed by solving the equation $a_{k} H\left(a_{k}\right)=a_{0} k$ for $a_{k}$, where $H=(\rho / 3)^{1 / 2} / \bar{m}$ as per equation (A1). In other words, $a_{k}$ is determined by following the dotted diagonal in Figure 3 from the point $(a, \rho)=\left(a_{0} k / H_{0}, \rho\left(a_{0}\right)\right)$ up to the left until it intersects the inflation curve. The current horizon wavenumber $k=H_{0}$ thus gives $a_{k}=a_{\text {exit }}$ (open triangle) and larger $k$ give larger $a_{k}$ (open square and circle). During the observable part $(N \sim 55)$ of slow-roll inflation, $H$ rarely drops by more than about a percent per e-folding, so for cosmologically observable scales the approximation $H\left(a_{k}\right) \approx H\left(a_{\text {exit }}\right)$ is quite accurate, giving simply $a_{k}=a_{0} k / H\left(a_{\text {exit }}\right)$ and thus

$$
\delta_{H}^{T}(k) \approx \sqrt{\frac{8 \rho\left(\frac{a_{\text {exit }}}{H e x i t} k\right)}{75 \pi^{2} \bar{m}^{4}}} .
$$

This has a simple geometric interpretation in terms of Figure 3. Since $\delta_{H}^{T} \propto \rho^{1 / 2}$ and $k \propto a_{k}$, the curve $\delta_{H}^{T}(k)$ in a log-log plot is simply the $\rho(a)$ curve from Figure 3 compressed by a factor of two vertically. In other words, $\delta_{H}^{T}(k)$ and $\rho(a)$ are the exact same curve, merely with different axis labeling.

This means that tensor spectral index

$$
n_{t} \equiv \frac{d \ln \delta_{H}^{T^{2}}}{d \ln k} \approx \frac{d \ln \delta_{H}^{T^{2}}}{d \ln a}=\frac{d \ln \rho}{d \ln a}
$$

is simply the slope of the $\rho(a)$-curve in Figure 3, evaluated at $a_{\text {exit }}$ (open triangle).

\section{Density fluctuations}

None of the three above-mentioned observables $\Omega_{k}$, $\rho_{\Lambda}(a)$ and $\delta_{H}^{T}(k)$ depended explicitly on the physics of inflation, being computable directly from the curve $\rho(a)$. We will now see that this is in many cases true even for the fourth observable, the density fluctuations $\delta_{H}(k)$.

$$
\text { a. Computing } \rho(a) \text { and } \delta_{H}(k) \text { from } V(\phi)
$$

The cosmic density is given by [11]

$$
\rho=V(\phi)+\frac{1}{2}|\dot{\phi}|^{2}+\rho_{\gamma \mathrm{mk}}
$$

and the evolution of the inflation vector is governed by [11]

$$
\ddot{\phi}+3 H \dot{\phi}+\nabla V(\phi)=0 .
$$

(For a review of inflation generalizations with nonstandard kinetic terms, see [11].) We ignore the $\rho_{\gamma \mathrm{mk}}{ }^{-}$ term in equation (A13) during inflation since it rapidly becomes negligible, having a steeper slope $(\leq-2)$ than that of $\rho(>-2)$ in Figure 3 .

The slow-roll approximation (SRA) is that the $\dot{\boldsymbol{\phi}}$-term in equation (A13) and the $\ddot{\boldsymbol{\phi}}$-term in equation (A14) are negligible, which gives

$$
\begin{gathered}
\rho=V, \\
\dot{\phi}=-\frac{\nabla \phi}{3 H} .
\end{gathered}
$$


Using equation (A1) and the identity $\partial / \partial t=$ $H \partial / \partial \ln a=-H \partial / \partial N$, this becomes

$$
\frac{\partial \phi}{\partial N} \approx \bar{m}^{2} \nabla \ln V
$$

In other words, the slow-rolling inflaton vector evolves just like a particle moving in an extremely viscous medium, so that the velocity is proportional to the force, and it always rolls in the direction of the gradient, i.e., takes the steepest descent. The only differences are that the time variable is $-N$ rather than $t$ and that the potential is $\ln V$ rather than $V$. For the 1-dimensional case, equation (A17) is usually integrated as

$$
N \equiv \ln \frac{a}{a_{\text {end }}}=\frac{1}{\bar{m}^{2}} \int_{\phi}^{\phi_{\text {end }}} \frac{V(\phi)}{V^{\prime}(\phi)} d \phi .
$$

If valid, the SRA remains valid as long as the slow-roll parameters $\epsilon \ll 1$ and $|\eta| \ll 1^{21}$, where

$$
\epsilon \equiv \frac{\bar{m}^{2}}{2}|\nabla \ln V|^{2}, \quad \eta \equiv \bar{m}^{2} \frac{V^{\prime \prime}}{V} .
$$

For the multidimensional case, $V^{\prime \prime}$ denotes the second derivative in the direction in which the field is rolling, i.e., in the direction of the gradient $\nabla \phi$. For the 1-dimensional case, the third slow-roll parameter is defined as

$$
\xi_{2} \equiv \bar{m}^{4} \frac{V^{\prime}(\phi) V^{\prime \prime \prime}(\phi)}{V(\phi)^{2}}
$$

For the $d$-dimensional case (where the vector $\phi$ has $d$ components), the power spectrum of density fluctuations $\delta_{H}(k)$ is given by $[10,11,129-131]$

$$
\delta_{H}(k)^{2}=\frac{\rho}{75 \pi^{2} \bar{m}^{2}} \sum_{i=1}^{d}\left(\frac{\partial N}{\partial \phi_{i}}\right)^{2},
$$

where the right hand side is evaluated at the exit epoch $a_{k}$ as usual, and $N(\phi)$ denotes the number of e-foldings for the field to roll from $\phi$ down to some fixed reference density according to equation (A17). Heuristically, equation (A21) is easy to understand: quantum fluctuations in $\phi$ are of order $\delta \phi \sim H \sim \rho^{1 / 2} / \bar{m}$, and if such a fluctuation in some particular spatial region delays the roll down to our current epoch by an amount $\delta N \sim \frac{\partial N}{\partial \phi} \delta \phi$, this will appear an an overdensity of order $\delta N$. Fluctuations from the different components $\phi_{i}$ of the inflaton

${ }^{21}$ For the multidimensional case, $|\eta| \ll 1$ is strictly speaking not a sufficient condition. The second derivative $\ddot{\phi}$ can get large either because the inflaton starts rolling faster (large $|\eta|$ so that $|\nabla \ln V|$ grows) or because it changes direction (a large eigenvalue of the Hessian matrix $(\ln V)_{, i j}$ corresponding to an eigenvector misaligned with the gradient). Generically, such eigenvectors will have a component in the gradient direction as well and cause a large $|\eta|$, so it requires some fine-tuning to fool the $|\eta|$ and $\epsilon$ tests. vector $\phi$ add in quadrature to give the total variance $\delta_{H}(k)^{2}$. Choosing our coordinates in $\phi$-space so that the field is momentarily rolling in the $\phi_{1}$-direction, we have $\partial N / \partial \phi_{1}=\left[\bar{m}^{2} \partial \ln V / \partial \phi_{1}\right]^{-1}=\left(2 \bar{m}^{2} \epsilon\right)^{-1 / 2}$, so

$\delta_{H}(k)^{2}=\frac{\rho}{75 \pi^{2} \bar{m}^{4}}\left[\frac{1}{2 \epsilon}+\frac{1}{\bar{m}^{2}} \sum_{2=1}^{d}\left(\frac{\partial N}{\partial \phi_{i}}\right)^{2}\right] \geq \frac{\rho}{150 \pi^{2} \bar{m}^{4} \epsilon}$,

In most studied cases, the walls of the multidimensional gorge in which the inflaton slowly rolls are much steeper than the roll direction, rendering the inequality of equation (A22) close to an equality [10, 11, 129-131].

\section{b. Computing $\delta_{H}(k)$ from $\rho(a)$}

Let us now eliminate $V(\phi)$ and rewrite the above quantities in terms of the $\rho(a)$ alone for the one-dimensional case. Letting primes ' denote $\partial / \partial N=-\partial / \partial \ln a$, we obtain

$$
\epsilon=\frac{1}{2}(\ln \rho)^{\prime}
$$

$$
\eta=(\ln \rho)^{\prime}+\frac{(\ln \rho)^{\prime \prime}}{2(\ln \rho)^{\prime}}
$$

$$
\delta_{H}(k)=\sqrt{\frac{\rho}{75 \pi^{2} \bar{m}^{4}(\ln \rho)^{\prime}}},
$$

again evaluated at the exit epoch $a_{k}$. This gives

$$
\begin{gathered}
n_{t}=\frac{\partial \ln \delta_{H}^{T^{2}}}{\partial k}=(\ln \rho)^{\prime}=2 \epsilon \quad(\mathrm{A} 27) \\
n_{s}-1=\frac{\partial \ln \delta_{H}^{2}}{\partial k}=-\left[\ln \rho-\ln (\ln \rho)^{\prime}\right]^{\prime}=\frac{(\ln \rho)^{\prime \prime}}{(\ln \rho)^{\prime}}-(\ln \rho)^{\prime} \\
\alpha=\frac{\partial n_{s}}{\partial \ln k}=(\ln \rho)^{\prime}+\frac{(\ln \rho)^{\prime \prime 2}}{(\ln \rho)^{\prime 2}}-\frac{(\ln \rho)^{\prime \prime \prime}}{(\ln \rho)^{\prime}} \quad(\mathrm{A} 29)
\end{gathered}
$$

In deriving these and related relations, the following identities are useful (for brevity, we here - and only here - we use the shorthand notation ${ }^{\circ}=\frac{d}{d \phi}$ and and $r=\ln \rho=\ln V)$ :

$$
\begin{aligned}
& \partial_{N}=-\bar{m}^{2} \dot{r} \partial_{\phi} \\
& \partial_{N}^{2}=\bar{m}^{4}\left(\dot{r}^{2} \partial_{\phi}^{2}+\dot{r} \ddot{r} \partial_{\phi}\right) \\
& \partial_{N}^{3}=-\bar{m}^{6}\left[\dot{r}^{3} \partial_{\phi}^{3}+3 \dot{r}^{2} \ddot{r} \partial_{\phi}^{2}+\left(\dot{r} \ddot{r}^{2}+\dot{r}^{2} \dddot{r}\right) \partial_{\phi}\right]
\end{aligned}
$$


etc. The derivatives of a curve $(\ln a, \ln \rho)=(-N, r)$ therefore satisfy

$$
\begin{aligned}
r^{\prime} & =-\bar{m}^{2} \dot{r}^{2} \\
r^{\prime \prime} & =2 \bar{m}^{4} \dot{r}^{2} \ddot{r} \\
r^{\prime \prime \prime} & =-\bar{m}^{6} \dot{r}^{2}\left(2 \ddot{r}^{2}+\dot{r} \dddot{r}\right) \\
r^{\prime \prime \prime \prime} & =-2 \bar{m}^{8} \dot{r}^{2}\left(4 \ddot{r}^{3}+7 \dot{r} \dddot{r} \dddot{r}+\dot{r}^{2} \dddot{r}\right) \\
\dot{r} & =\frac{\sqrt{-r^{\prime}}}{\bar{m}} \\
\ddot{r} & =-\frac{1}{2 \bar{m}^{2}} \frac{r^{\prime \prime}}{r^{\prime}} \\
\dddot{r} & =-\frac{1}{\bar{m}^{3}\left(-{r^{\prime}}^{3 / 2}\right)} \frac{r^{\prime \prime \prime}+r^{\prime \prime 2}}{2 r^{\prime}}
\end{aligned}
$$

\section{APPENDIX B: INFLATION NEAR PEAKS AND TROUGHS}

Since the cosmological parameter predictions for inflation with $m_{h} \gg \bar{m}$ or $m_{h} \ll \bar{m}$ depend only on the behavior of the inflaton potential near its minima or maxima, respectively, let us review the analytic solutions for these two important cases and compute the predicted cosmological parameter probability distributions.

\section{Inflation near a minimum}

Taylor expanding $V(\phi)$ to second order around a minimum $\phi_{0}$ gives

$$
V(\phi)=V\left(\phi_{0}\right)+\frac{1}{2} V^{\prime \prime}\left(\phi_{0}\right)\left(\phi-\phi_{0}\right)^{2},
$$

where, generically, $V^{\prime \prime}(\phi)>0$. Since we saw in Section VI that only those minima where $\left|\rho_{\Lambda}\right|=\left|V\left(\phi_{0}\right)\right|$ was negligibly small contributed significantly to the parameter probability distribution, we need only consider the simple case $V\left(\phi_{0}\right)=0$, i.e., the standard parabolic potential case [32]. Using equations (A19), this gives

$$
\epsilon=\eta=\frac{2 \bar{m}^{2}}{\left(\phi-\phi_{0}\right)^{2}}
$$

so inflation ends with $\epsilon=\eta=1$ when $\phi$ has rolled down to

$$
\phi_{\mathrm{end}}=\phi_{0} \pm \sqrt{2} \bar{m} .
$$

Equation (A18) now gives equation (37), which in turn (via equations 11-20) gives the well-known results

$$
\begin{aligned}
N & =\frac{\left(\phi-\phi_{0}\right)^{2}}{4 \bar{m}^{2}}-\frac{1}{2}, \\
\phi & =\phi_{0} \pm \bar{m} \sqrt{2(2 N+1)} \\
\epsilon & =\eta=\frac{1}{2 N+1}, \\
\xi_{2} & =0,
\end{aligned}
$$

and the cosmological predictions of equations (37)-(43).

\section{Inflation near a maximum}

Taylor expanding $V(\phi)$ to second order around a maximum $\phi_{0}$ gives

$$
V(\phi)=V\left(\phi_{0}\right)\left[1+\frac{\eta_{0}}{2}\left(\frac{\phi-\phi_{0}}{\bar{m}}\right)^{2}\right],
$$

where $\eta_{0}$ is the second slow-roll parameter of equation (A19) evaluated at the maximum. Generically, $\eta_{0}<0$. Using equation (A19), this gives

$$
\begin{aligned}
& \epsilon=\frac{\eta_{0}^{2}\left(\phi-\phi_{0}\right)^{2}}{2 \bar{m}^{2}}\left[1+\frac{\eta_{0}}{2}\left(\frac{\phi-\phi_{0}}{\bar{m}}\right)^{2}\right]^{-2}, \\
& \eta=\eta_{0}\left[1+\frac{\eta_{0}}{2}\left(\frac{\phi-\phi_{0}}{\bar{m}}\right)^{2}\right]^{-2} .
\end{aligned}
$$

In the early stages of $\phi$ rolling off the peak, while the term in square brackets remains near unity, equation (A18) gives

$$
\left(\phi-\phi_{0}\right) \propto e^{\eta_{0} N},
$$

so at the observable epoch $N \sim 55$, the field is exponentially close to the peak unless $\eta_{0}$ happens to lie in the small interval $-0.02 \lesssim \eta_{0}<0$ (slow-roll requires $\left.-1<\eta_{0}<0\right)$. According to the 2nd order expansion of equation (B8), inflation ends only when $\left|\phi-\phi_{0}\right| \gtrsim \bar{m}$. However, the scaling relations given in Section $\mathrm{V}$ show that inflation ends much sooner because of higher order terms in the Taylor expansion, usually because the cubic term causes inflation to end when $|\eta|=1$ with $\left|\phi_{\text {end }}-\phi_{0}\right| \sim m_{h}{ }^{3} / \bar{m}^{2}$, which is borne out by the $m_{h} \ll \bar{m}$ simulations in Section V. For the observable regime $N \sim 55$, we thus have

$$
\begin{aligned}
\left|\phi-\phi_{0}\right| & \sim \frac{m_{h}^{3}}{\bar{m}^{2}} e^{\eta_{0} N}, \\
\frac{V^{\prime}(\phi)}{V(\phi)} & \sim \frac{m_{h}^{3} \eta_{0}}{\bar{m}^{4}} e^{\eta_{0} N} \approx 0, \\
\epsilon & \sim \frac{\eta_{0}^{2} m_{h}{ }^{6}}{\bar{m}^{6}} e^{2 \eta_{0} N} \approx 0, \\
\eta & \approx \eta_{0}, \\
\xi_{2} & =\bar{m}^{4} \frac{V^{\prime} V^{\prime \prime \prime}}{V^{2}} \sim \eta_{0} e^{\eta_{0} N} \approx 0,
\end{aligned}
$$

and the cosmological predictions of equations (48)-(54). In equation (B16), we used the scaling relation $V^{\prime \prime \prime} / V \sim$ $m_{h}{ }^{3}$ which follows from equation (32). 
[1] D. N. Spergel et al., ApJS, 148, 175 (2003)

[2] M. Tegmark et al., PRD, 69, 103501 (2004)

[3] U. Seljak et al., astro-ph/0407372, 2004

[4] A. Linde, PRD, 59, 23503 (1999)

[5] A. Linde, JCAP, 305, 2 (2003)

[6] A. Linde, JHEP, 111, 52 (2001)

[7] M. Barnard and A. Albrecht, hep-th/0409082, 2004

[8] A. D. Linde, Particle Physics and Inflationary Cosmology (Harwood: Switzerland, 1990)

[9] S. Dodelson, W. H. Kinney, and E. W. Kolb, PRD, 56, 3207 (1997)

[10] D. H. Lyth and A. A. Riotto, Phys. Rep., 314, 1 (1999)

[11] A. R. Liddle and D. H. Lyth, Cosmological Inflation and Large-Scale Structure (Cambridge Univ. Press: Cambridge, 2000)

[12] H. V. Peiris et al., ApJS, 148, 213 (2003)

[13] W. H. Kinney, E. W. Kolb, A. Melchiorri, and A. Riotto, hep-ph/0305130, 2003

[14] A. R. Liddle and A. J. Smith, PRD, 68, 061301 (2003)

[15] D. Wands, New Astron. Reviews, 47, 781 (2003)

[16] R. Bousso and J. Polchinski, JHEP, 6, 6 (2000)

[17] J. L. Feng, J. March-Russell, S. Sethi, and Wilczek F, Nucl. Phys. B, 602, 307 (2001)

[18] S. Kachru, R. Kallosh, A. Linde, and S. P. Trivedi, PRD, 68, 046005 (2003)

[19] L. Susskind, hep-th/0302219, 2003

[20] S. Ashok and M. R. Douglas, JHEP, 401, 60 (2004)

[21] S. Kachru, R. Kallosh, A. Linde, J. Maldacena, L. McAllister, and S. P. Trivedi, hep-th/0308055, 2003

[22] O. DeWolfe, S. Kachru, and H. Verlinde, JHEP, 405, 17 (2004)

[23] F. Denef and M. R. Douglas, JHEP, 405, 72 (2004)

[24] A. Giryavets and S. Kachru, JHEP, 408, 2 (2004)

[25] B. Freivogel and L. Susskind, hep-th/0408133, 2004

[26] J. Conlon and F. Quevedo, JHEP, 410, 39 (2004)

[27] O. DeWolfe, A. Giryavets, S. Kachru, and W. Taylor, hep-th/0411061, 2004

[28] N. Arkani-Hamed, S. Dimopoulos, and S. Kachru, hepth/0501082, 2005

[29] B. S. Acharya, F. Denef, and R. Valandro, hepth/0502060, 2005

[30] T. Banks, M. Dine, and E. Gorbatov, JHEP, 408, 58 (2004)

[31] T. Banks, hep-th/0412129, 2004

[32] A. D. Linde, Phys. Lett. B, 175, 395 (1986)

[33] D. Giulini, E. Joos, C. Kiefer, J. Kupsch, I. O. Stamatescu, and H. D. Zeh, Decoherence and the Appearance of a Classical World in Quantum Theory (Berlin: Springer, 1996)

[34] D. Polarski and A. A. Starobinsky, Class. Quant. Grav., 13, 377 (1996)

Authors: David Polarski, Alexei A. Starobinsky

[35] K. Kiefer and D. Polarski, Ann. Phys., 7, 137 (1998)

[36] A. R. Liddle and S. M. Leach, PRD, 68, 103503 (2003)

[37] L. Hui and S. Dodelson, PRL, 91, 131301 (2003)

[38] G. M. Hossain, gr-qc/0411012, 2005

[39] M. Hoffman and M. S. Turner, PRD, 64, 23506 (2001)

[40] W. H. Kinney, PRD, 66, 83508 (2002)

[41] A. R. Liddle, PRD, 68, 103504 (2003)

[42] U. Seljak, and M. Zaldarriaga, ApJ, 469, 437 (1996)

[43] N. Arkani-Hamed, P. Creminelli, S. Mukohyama, and M. Zaldarriaga, JCAP, 404, 1 (2004)

[44] J. Khoury, B. A. Ovrut, P. J. Steinhardt, and N. Turok, PRD, 64, $123522(2001)$

[45] J. Khoury, P. J. Steinhardt, and N. Turok, PRL, 91, 161301 (2003)

[46] A. Aguirre and M. Tegmark, hep-th/0409072, 2004

[47] N. Boström, Anthropic Bias: Observation Selection Effects in Science and Philosophy (Routledge: New York, 2002)

[48] A. H. Guth, Phys.Rep., 333, 555 (2000)

[49] A. H. Guth, astro-ph/0002188, 2000

[50] A. H. Guth, astro-ph/0404546, 2004

[51] M. Tegmark, astro-ph/0302131, 2003

[52] A. D. Linde and A. Mezhlumian, Phys. Lett. B, 307, 24 (1993)

[53] A. D. Linde, D. A. Linde, and A. Mezhlumian, Phys. Rev. D, 49, 1783 (1994)

[54] J. Garcia-Bellido, A. D. Linde, and D. A. Linde, PRD, 50, 730 (1994)

[55] J. Garcia-Bellido and A. D. Linde, PRD, 51, 429 (1995)

[56] A. Linde, D. Linde, and A. Mezhlumian, Phys. Lett. B, 345, 203 (1995)

[57] S. Winitzki and A. Vilenkin, PRD, 53, 4298 (1996)

[58] A. D. Linde and A. Mezhlumian, PRD, 53, 4267 (1996)

[59] A. D. Linde, D. A. Linde, and A. Mezhlumian, Phys. Rev. D, 54, 2504 (1996)

[60] A. Vilenkin and S. Winitzki, PRD, 55, 548 (1997)

[61] A. Vilenkin, $P R L, \mathbf{7 4}, 846$ (1995)

[62] A. Vilenkin, PRD, 27, 2848 (1983)

[63] B. Carter 1974, in IAU Symposium 63, ed. S. Longair (Reidel: Dordrecht)

[64] J. D. Barrow and F. J. Tipler, The Anthropic Cosmological Principle (Clarendon Press: Oxford, 1986)

[65] Y. V. Balashov, Am. J. Phys., 59, 1069 (1991)

[66] M. Tegmark, Ann. Phys., 270, 1 (1998)

[67] A. D. Linde 1987, in 300 Years of Gravitation, ed. S. Hawking and W. Israel (Cambridge University Press: Cambridge)

[68] S. Weinberg, PRL, 59, 2607 (1987)

[69] G. Efstathiou, $M N R A S, \mathbf{2 7 4}$, L73 (1995)

[70] H. Martel, P. R. Shapiro, and S. Weinberg, ApJ, 492, $29(1998)$

[71] J. Garriga and A. Vilenkin, PRD, 67, 43503 (2003)

[72] A. Vilenkin, PRL, 81, 5501 (1998)

[73] A. Vanchurin, A. Vilenkin, and S. Winitzki, PRD, 61, 83507 (2000)

[74] J. Garriga and A. A. Vilenkin, PRD, 64, 23507 (2001)

[75] M. Tegmark and M. Zaldarriaga, PRD, 66, 103508 (2002)

[76] M. Tegmark, Science, 296, 1427 (2002)

[77] A. D. Linde, hep-th/0211048, 2002

[78] S. Hannestad, PRD, 70, 43506 (2004)

[79] S. Kachru, private communication (2004)

[80] A. Guth, PRD, 23, 347 (1981)

[81] A. A. Starobinsky, Phys. Lett. B, 91, 99;1980 (1980)

[82] A. D. Linde, Phys. Lett. B, 108, 389 (1982)

[83] A. Albrecht and P. J. Steinhardt, Phys. Rev. Lett., 48, 1220 (1982)

[84] A. D. Linde, Phys. Lett. B, 129, 177 (1983)

[85] D. J. Eisenstein, W. Hu, and M. Tegmark, ApJ, 518, 2 (1999) 
[86] M. Tegmark, M. Blanton, M. A. Strauss, F. S. Hoyle, D. Schlegel, R. Scoccimarro, M. S. Vogeley, D. H. Weinberg, I. Zehavi et al., ApJ, 606, 702 (2004)

[87] M. Tegmark and M. J. Rees, ApJ, 499, 526 (1998)

[88] M. Tegmark, A. Vilenkin, and L. Pogosian, astro$\mathrm{ph} / 0304536,2003$

[89] L. Pogosian, A. Vilenkin, and M. Tegmark, JCAP, 407, 5 (2004)

[90] W. H. Press and P. Schechter, ApJ, 187, 425 (1974)

[91] J. Binney, ApJ, 215, 483 (1977)

[92] M. J. Rees and J. P. Ostriker, MNRAS, 179, 541 (1977)

[93] J. Silk, ApJ, 211, 638 (1977)

[94] S. D. M White and M. J. Rees, MNRAS, 183, 341 (1978)

[95] R. K. Sheth and G. Tormen, MNRAS, 308, 119 (1999)

[96] F. Wilczek, hep-ph/0408167, 2004

[97] A. Aguirre, PRD, 64, 83508 (2001)

[98] M. L. Graesser, S. D. Hsu H, A. Jenkins, and M. B. Wise, Phys. Lett. B, 600, 15 (2004)

[99] Q. HuangG and Li M, hep-th/0410095, 2004

[100] A. Aguirre and S. Gratton, PRD, 65, 83507 (2002)

[101] A. Aguirre and S. Gratton, PRD, 67, 83515 (2003)

[102] A. Albrecht and L. Sorbo, hep-th/0405270, 2004

[103] A. A. Starobinsky, Fundamental Interactions (MGPI Press, Moscow: p.55, 1984)

[104] A. A. Starobinsky 1986, in Current Topics in Field Theory, Quantum Gravity and Strings, Lecture Notes in Physics, vol. 246, ed. H. J. de Vega H J and N. Sanchez (Springer: Heidelberg.)

[105] A. S. Goncharov, A. D. Linde, and V. F. Mukhanov, Int. J. Mod. Phys. A, 2, 561 (1987)

[106] D. S. Salopek and J. R. Bond, PRD, 43, 1005 (1991)

[107] A. Borde, A. Guth, and A. Vilenkin, PRL, 90, 151301 (2003)

[108] A. Vilenkin, gr-qc/0409055, 2004
[109] P. J. Steinhardt, G. W. Gibbons, S. W. Hawking, and S. T. C Siklos, 1983, Cambridge University Press;Cambridge (The Very Early Universe)

[110] R. A. Alpher and R. C. Herman, Rev. Mod. Phys., 22, 153 (1950)

[111] S. Coleman and F. de Luccia, PRD, 21, 3305 (1980)

[112] L. Smolin, hep-th/0407213, 2004

[113] J. R. Bond, L. Kofman, and D. Pogosyan, Nature, 380, 603 (1996)

[114] J. Garriga and A. Vilenkin, PRD, 57, 2230 (1998)

[115] J. E. Carlstrom, J. Kovac, E. M. Leitch, and C. Pryke, astro-ph/0308478, 2003

[116] A. de Oliveira-Costa, astro-ph/0406358, 2004

[117] J. Khoury, P. J. Steinhardt, and N. turok, PRL, 91, 161301 (2003)

[118] D. Babich, P. Creminelli, and M. Zaldarriaga, JCAP, 0408, 009 (2004)

[119] A. G. Riess et al., astro-ph/0402512, 2004

[120] A. G. Riess et al., Astron. J., 116, 1009 (1998)

[121] S. Perlmutter et al., ApJ, 517, 565 (1999)

[122] Y. Wang and M. Tegmark, PRL, 92, 241302 (2004)

[123] Y. Wang and M. Tegmark, astro-ph/0501351, 2005

[124] C. Gordon and W. Hu, PRD, 70, 083003 (2004)

[125] R. Donagi, Y. He, B. A. Ovrut, and R. Reinbacher, hepth/0405014, 2004

[126] J. Garriga and A. Vilenkin, PRD, 64, 043511 (2001)

[127] S. Dimopoulos and Lawrence. J. Hall, Phys. Lett. B, 196, 135 (1987)

[128] S. Hannestad, PRD, 70, 043506 (2004)

[129] A. A. Starobinsky, JETP Lett., 42, 152 (1985)

[130] D. S. Salopek, PRD, 52, 5563 (1995)

[131] M. Sasaki and E. D,. Prog. Theor. Phys. Stewart, 95, 71 (1996) 